\title{
Male sexual violence victimization: Definitions, epidemiological profile, and psychological impact
}

Ekta Choudhary

West Virginia University

Follow this and additional works at: https://researchrepository.wvu.edu/etd

\section{Recommended Citation}

Choudhary, Ekta, "Male sexual violence victimization: Definitions, epidemiological profile, and psychological impact" (2009). Graduate Theses, Dissertations, and Problem Reports. 4450. https://researchrepository.wvu.edu/etd/4450

This Dissertation is protected by copyright and/or related rights. It has been brought to you by the The Research Repository @ WVU with permission from the rights-holder(s). You are free to use this Dissertation in any way that is permitted by the copyright and related rights legislation that applies to your use. For other uses you must obtain permission from the rights-holder(s) directly, unless additional rights are indicated by a Creative Commons license in the record and/ or on the work itself. This Dissertation has been accepted for inclusion in WVU Graduate Theses, Dissertations, and Problem Reports collection by an authorized administrator of The Research Repository @ WVU.

For more information, please contact researchrepository@mail.wvu.edu. 


\title{
Male Sexual Violence Victimization: \\ Definitions, Epidemiological Profile, and Psychological Impact
}

\author{
Ekta Choudhary
}

Dissertation submitted to the

School of Medicine

at West Virginia University

in partial fulfillment of the requirements for the degree of

Doctor of Philosophy
in
Public Health Sciences

Robert M. Bossarte, PhD

S. Suresh Madhavan, MBA, PhD

Jim Helmkamp, PhD

Amy Fiske, PhD

Michael Hendryx, PhD

Department of Community Medicine

Morgantown, West Virginia

2009

Keywords: Sexual Violence Victimization, Males, Epidemiological Profile, Psychological

Impact, Aggressive Behaviors, Suicide Ideation, Deliberate Self-harm, Definitions 


\section{ABSTRACT \\ Male Sexual Violence Victimization: Definitions, Epidemiological Profile, and Psychological Impact}

Ekta Choudhary

Sexual violence or sexual assault refers to any forcible or non-forcible sexual activity where consent is not obtained or freely given. In comparison to female victims, there is a relative paucity of literature examining the characteristics and adverse health outcomes associated with male sexual assault as well as long-term consequences. Past studies have relied primarily on population-based survey data to estimate the prevalence of sexual assault and associated health outcomes. The main objective of this study was to enhance our understanding of male sexual assault through results obtained from three related projects. In the first study, data from the 2001-2005 National Incident Based Reporting System (NIBRS) was used to construct an epidemiological profile of sexual assault among males. Using the NIBRS data for multiple years, significant age- and race-specific time-trends were observed among males. Results of this study indicated that despite a higher rate of sexual assault among young males, sexual assault incidents were reported by men of all age groups. In the second study, self-administered survey was used to understand the psychological impact of sexual violence victimization among males. The results of this study support previous findings that sexual victimization is associated with a range of health risk behaviors and negative psychological consequences among males. In addition, this study adds new information about the associations between sexual victimization and aggressive behaviors among males, including the co-occurrence of risk behavior such as alcohol and substance abuse. Finally, the findings of the third study also highlighted some important differences and similarities in the description of unwanted sexual experience among males and females. In comparisons to women, relatively little work has been done in the area of male sexual assault. For better understanding of different aspects of male sexual assault, more comprehensive and multidisciplinary approaches are needed. This study also demonstrated the importance of investigating male sexual assault using various data sources. Strong preliminary evidence has emerged from this study that supports and extends previous research and establishes the need for future in-depth investigations of the behavioral and psychological consequences of sexual violence victimization. 


\section{ACKNOWLEDGEMENTS}

I owe my deepest gratitude to my advisor Dr. Robert M. Bossarte, whose encouragement, supervision, and support helped me in executing this arduous task from its conception to its completion. Over the last three years he played various roles in my professional life, he was not only a mentor, but also a critic, a counselor, a motivator, a role model and above all a great friend. Thank you Dr. Bossarte.

I extend my thanks to my co-advisor Dr. S. Suresh Madhavan for his invaluable assistance and precious guidance. His invaluable advice and support kept me on-course and focused on my project.

It's my pleasure to thank my committee members, Dr. Jim Helmkamp, Dr. Amy Fiske, and Dr. Michael Hendryx, for their advice and support. A special thank goes to Dr. Amy Fiske for her help with the survey study.

I would also like to thank Dr. Alan Ducatman, Chairman and faculty members at the Department of Community Medicine for being supportive during my tenure as a graduate student. I am thankful to Dr. Keith Zullig, Director of the PhD Program in the Public Health Sciences, for his support. My special thanks go to Dr. Rob Pack, for his encouragement and friendship during the first year of the PhD program. My sincere thanks go to the staff and faculty members at the Injury Control Research Center and Center for Rural Emergency Medicine.

I am indebted to my fellow graduate students at the Department of Community Medicine for their friendship and continuous support over the years. My special thanks to "the ladies of the first cohort": Penney, Traci, Stephanie, and Danielle for their friendship and lending a helping hand when needed. I also like to thank John Blosnich for his friendship and help.

I am also thankful to my family, my parents, my sisters and my brother for their unconditional love and encouragement. I wish to thank my extended family for their support.

Above all, I want to thank my husband Sushant for his love and enjoying all the sweet and sour moments with me. His support and love kept me going 


\section{TABLE OF CONTENTS}

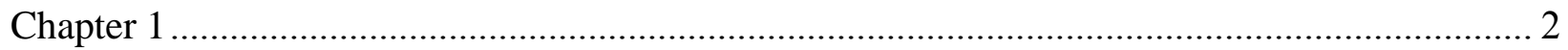

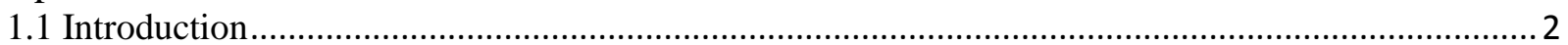

1.1.1 Prevalence of Male Sexual Assault .......................................................................................... 3

1.1.2 Perpetrator and Incident Characteristics of Sexual Violence ............................................... 6

1.1.3 Injuries Sustained by Victims of Sexual Violence ...................................................................... 7

1.1.4 Psychological Consequences of Sexual Violence ................................................................ 8

1.1.5 Externalized Behaviors among Male Victims ...................................................................... 10

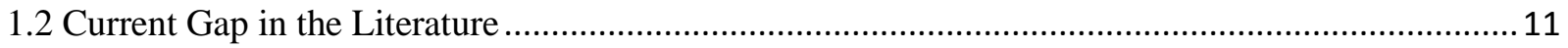

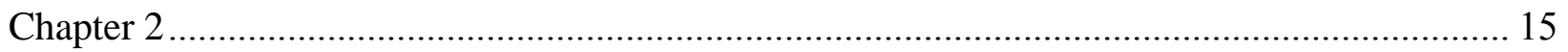

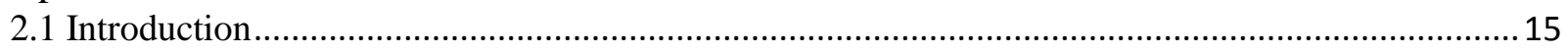

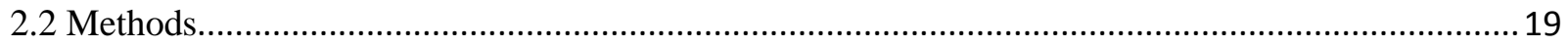

2.2.1 Data

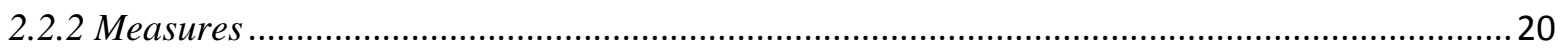

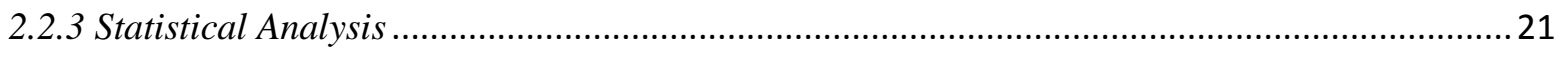

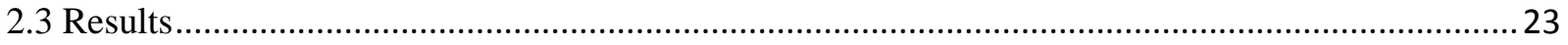

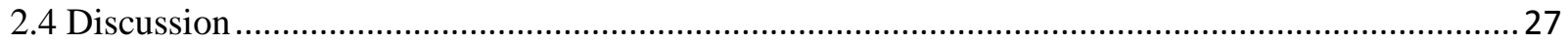

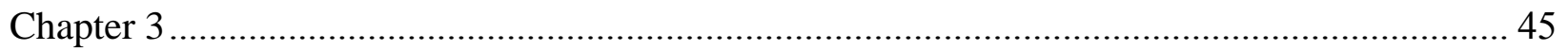

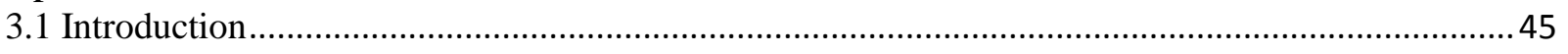

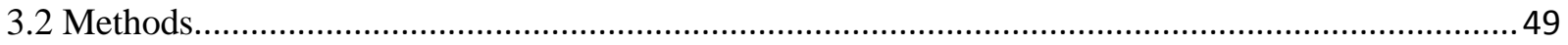

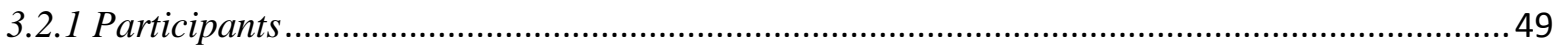

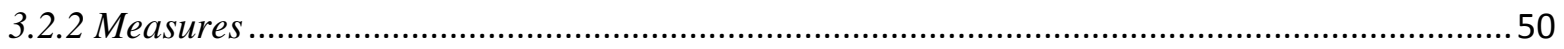

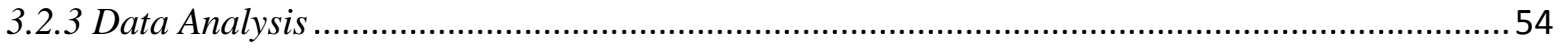

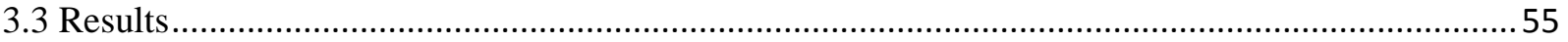

3.3.1 Prevalence of Risk Behaviors, Negative Health Outcomes, and Sexual Victimization among College-attending Males and Females...................................................................................... 55

3.3.2 Associations between Aggressive Behaviors and Sexual Victimization among Males .............. 58

3.3.3 Associations between Suicidal Behavior, DSH, and Sexual Violence among Victimized Males

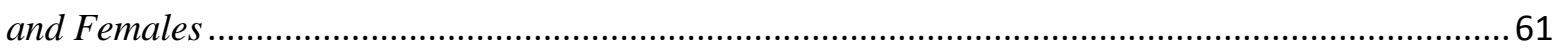

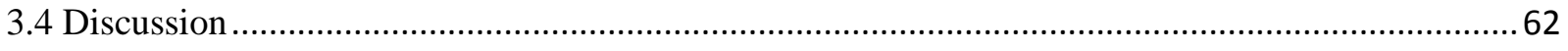

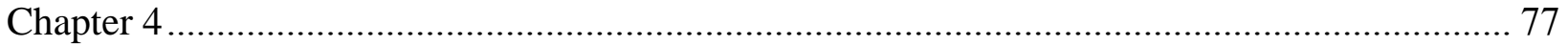

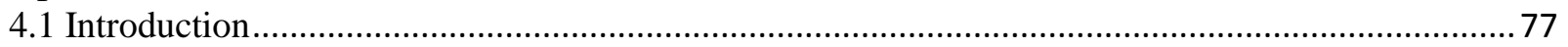




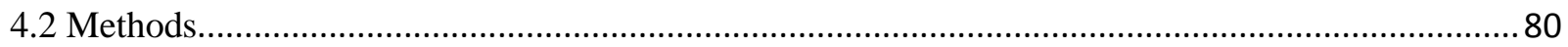

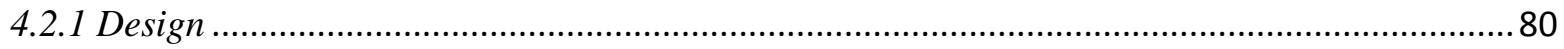

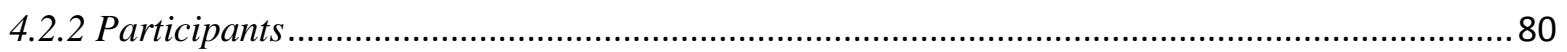

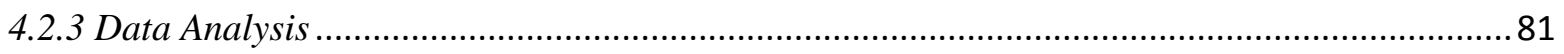

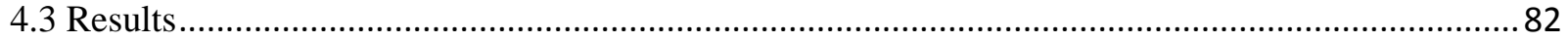

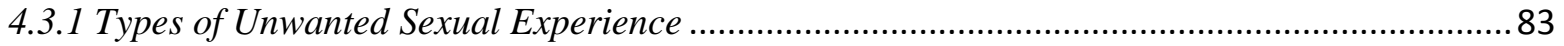

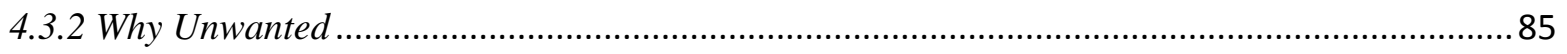

4.3.3 Emotions or Feelings Expressed towards Unwanted Sexual Experience ................................ 88

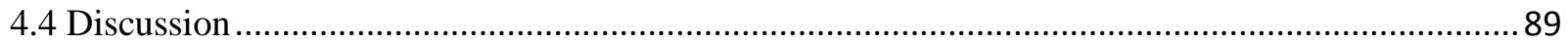

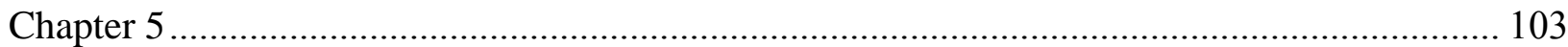

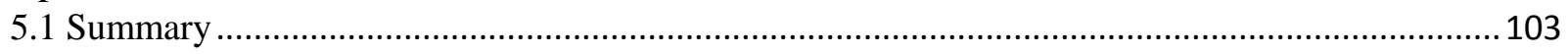

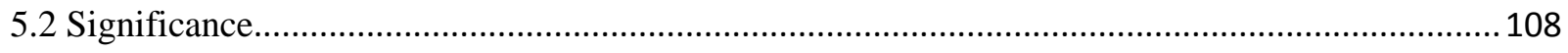

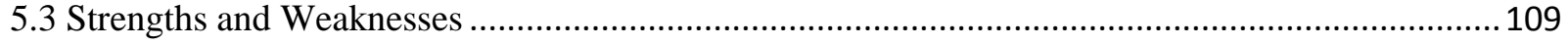

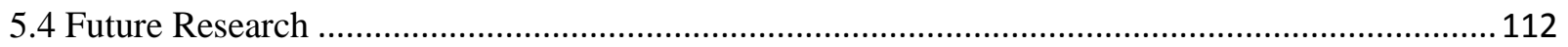

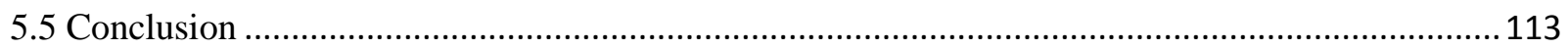

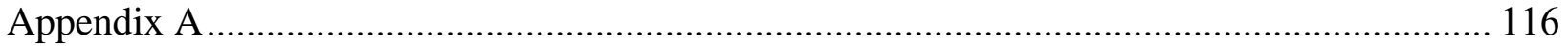

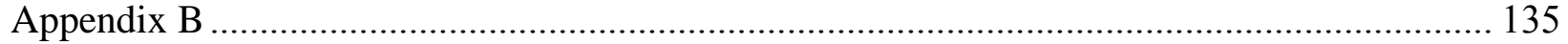

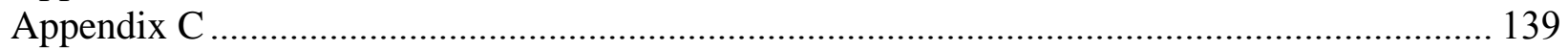

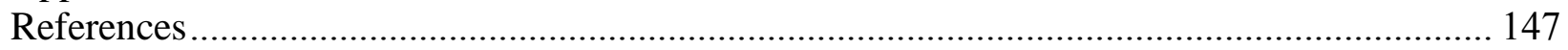


Chapter 1 


\section{Chapter 1}

\subsection{Introduction}

Sexual victimization (SV) refers to sexual activity where consent is not obtained or freely given. The Centers for Disease Control and Prevention's (CDC) National Center for Injury Prevention and Control (NCIPC) developed the Sexual Violence Surveillance: Uniform Definitions and Recommended Data Elements (Version 1.0), which defined sexual violence as "any sexual act that is forced against someone's will. These acts can be physical, verbal, or psychological. ${ }^{1 \text { " }}$ The National Center for Victims of Crime (www.ncvc.org) has suggested that sexual assault can take many forms ranging from use of physical force such as rape or attempted rape to any unwanted sexual contact, coercion, or threat. Thus, acts that fall under the broad category of sexual assault include forced sexual intercourse (rape), sodomy (oral or anal sexual acts), child molestation, incest, fondling and attempted rape. The American Medical Association provided a legal definition of sexual assault as "any genital, oral or anal penetration by a part of the accused's body or by an object, using force or without the victim's consent. ${ }^{2}$ A recent review of literature suggested that "sexual assault" or "sexual violence" has been used by researchers to describe a complete range of sexual acts which includes forced touching or kissing; verbally coerced intercourse; and physically forced vaginal, oral or anal penetration. ${ }^{3}$ In the present study, the terms "sexual violence" and "sexual assault," suggesting various types of unwanted sexual experiences, will be used interchangeably.

It has been estimated that sexual violence victimization affects approximately 3 million men and 18 million women in the United States. ${ }^{4,5}$ Among the few studies that have considered the impact of sexual violence on males, the focus have primarily been on childhood sexual abuse. In general, existing studies have drawn data from small samples, mainly from males who attend 
counseling services. In comparison to female victims, there is a relative paucity of research on the correlates and consequences of male sexual assault. ${ }^{6,7}$

Our long-term goal is to increase knowledge about adverse mental health consequences associated with male sexual assault victimization, which, in turn, will help to inform the development of prevention and intervention programs. The main objective of this project is to enhance our current understanding of male sexual assault through studies of the epidemiological characteristics, mental health and victimization, and gender-based differences in definitions of male sexual assault. In the first study we will estimate the rate of sexual assault and explored the characteristics of sexual assault incidents, victims, and perpetrators using data from a large criminological incident-based reporting system. Second, we will characterize the prevalence of sexual violence and identify significant differences in the correlated risk behaviors (such as binge drinking, substance abuse, and violence-related behavior) among males. Finally, we will explore differences in definitions of sexual assault among a sample of young adults attending college. Given prevailing societal beliefs and notions of traditional gender roles (i.e., that men cannot be the victims of sexual assault, that men who are sexually assaulted by other men must be gay, or that men are less affected by sexual assault than women), ${ }^{8-10}$ it is important to understand how males and females define sexual assault.

\subsubsection{Prevalence of Male Sexual Assault}

Previous studies have suggested that one in six U.S women and one in thirty-three U.S. men will experience an attempted or completed rape at some point in their lifetime. ${ }^{4}$ In comparison to females, the prevalence and characteristics of male sexual assault in the general population are not well documented. ${ }^{11}$ Current studies do not provide extensive information on male sexual violence victimization, however some have reported that sexual assault against males may be 
more prevalent than previously thought. ${ }^{6}$ In 2006, the National Crime Victimization Survey (NCVS) estimated 27,970 (20 per 100,000 persons) attempted/completed sexual assaults of U.S. males age 12 and over. The National Violence Against Women Survey (NVAWS) estimated 111,298 attempted/completed rapes against men age 18 and older in 1995; nearly six times greater than 1995 NCVS estimates of 19,388 attempted/completed rapes of U.S. males, 12 and older. ${ }^{4}$ Various other studies have estimated the prevalence of male sexual violence to vary between 0.6-22.2\% (depending upon the type of assault and population investigated), notably lower than estimates of female victimization (estimated 15 to $25 \%$ females in the general population). ${ }^{4,12,13}$ Tjaden and Thoennes (2006) estimated that approximately $4.5 \%$ of males in the U.S. population have experienced a sexual assault, whereas a study by Stermac et al. (1996) reported that approximately $7 \%$ of males in the general U.S. population have experienced some form of sexual violence.

Increased risk for sexual assault victimization may be increased for certain groups. Sexual assault among incarcerated males is comparatively well studied, however, the generalizability of findings from these studies to males in the general population is not clear. ${ }^{14,15}$ - Past studies have suggested that gay and/or bisexual males are more likely to become victims of sexual violence. ${ }^{16}$, 17 Of a sample of 358 gay or bisexual men between the age of 19-30 years, $14 \%$ reported nonconsensual sex after the age of $14 .{ }^{18}$ Another recent study based on a random sample of U.S. men reported that a majority of men were victims of childhood sexual abuse. ${ }^{11}$ Various clinical studies have estimated the percentage of male victims of sexual violence to be between $6-12 \% .{ }^{19-}$ 21

A review of literature by Isely (1998) indicated that the prevalence of sexual violence victimization is higher among college-attending males (average age 20 years) when compared to 
other age groups. ${ }^{4}$ Another study looking at the prevalence of male sexual assault in the college student population suggested that $14 \%$ of males (compared with $24 \%$ females) had experienced an unwanted sexual experience at least once in their lives. ${ }^{22}$ Most studies of sexual assault among college populations have been primarily designed to study female sexual assault and a majority of these studies did not use consistent definitions of sexual assault. Results from a survey of college-age men and women reported that $34 \%$ of men in a sample of university students experienced at least one unwanted sexual experience at age of 17 or older. ${ }^{23}$ A study of aggression and sexual victimization among university students by Lott et al. (1982) reported that $5 \%$ of men had experienced unwanted sexual touching while enrolled in college. ${ }^{24}$ Another study of college-aged men by Aizenman \& Kelley (1988) reported that $6 \%$ of males in their sample had experienced rape perpetrated by an acquaintance and $17 \%$ experienced unwanted sexual contact. ${ }^{25}$ Another study of both undergraduate and graduate students reported that $18.5 \%$ of males reported some form of sexual coercion ranging from unwanted participation in sex play to forced sexual intercourse. ${ }^{26}$ Smyth et al. (2008) examined the prevalence, nature, severity, and disclosure of adverse experiences among undergraduate students from diverse academic settings. ${ }^{27}$ Findings from their study suggested that $12.3 \%$ of total male participants $(1,695)$ had experienced an adverse sexual event at some point in their lifetime. While these studies provide some information on the prevalence of sexual assault among college attending males, differences in definitions of sexual assault, a lack of specificity surrounding different categories of sexual violence, and possible differences in definitions of sexual assault may limit generalizability of these efforts. 


\subsubsection{Perpetrator and Incident Characteristics of Sexual Violence}

Few existing studies have simultaneously examined perpetrator and incident characteristics of male sexual assault. Findings from NVAWS suggested that most male victims tend to be sexually assaulted by acquaintances, such as friends, teachers, co-workers, or neighbors and only $22.8 \%$ of all male victims were assaulted by a stranger. ${ }^{4}$ The results of this study also suggested that the majority of the perpetrators of male sexual assault were males. Hillman et al. (1990) reported that $75 \%$ of the male victims were assaulted by more than one perpetrator. ${ }^{28}$ Walker et al. (2005) reported that someone known to the victim was responsible for most of the assaults, but strangers also carried out a significant number. ${ }^{21}$ Of the incidents involving a female perpetrator, most assailants were known to the victim. ${ }^{14,29}$ A review of emergency department data by Pesola et al. (1999) ${ }^{30}$ reported that adult men were more likely to be assaulted by someone they knew than females. Estimates from a nationally representative sample of male and female victims of sexual assault suggested that approximately $91 \%$ of male victims reported that their perpetrator used a weapon and $34 \%$ of all male victims were also physically assaulted. ${ }^{4}$

Findings from clinical studies suggest that a large number of incidents occurred in a residential place (either perpetrator's or victim's home). ${ }^{19,21,28}$ Findings from a population-based survey indicated that the majority of victims were assaulted in private rather than public settings and alcohol and drugs may have played an important role in the assault ${ }^{4}$. About two thirds of males who were sexually assaulted reported that either they or their assailant was using drugs and/or alcohol at the time of the incident. ${ }^{4}$ Male victims reported sexually abusive acts ranging from verbal coercion to forced sodomy ${ }^{13,25,29,31}$ and more severe types of sexual assault, specifically forced anal penetration. ${ }^{19,20,28}$ 
It is possible that most healthcare professionals and researchers view victimization of a male by a female perpetrator as conceptually challenging and physically implausible. One study of the perception of sexual assault found that male victims of sexual assault were more likely to be blamed for their victimization especially when the perpetrator was a female. ${ }^{22}$ Gender role beliefs (e.g., men should always be available to women) serve to minimize the detriment of sexual assault of men by women. ${ }^{22,32}$ Thus, it is likely that the gender of the perpetrator has a different psychological impact on male victims. Men are more likely to report higher levels of homophobia than women ${ }^{33,34}$ and tend to blame male victims more than women do. ${ }^{7,35}$ Males are also more likely to blame themselves when the perpetrator is a female, especially in cases where they responded physiologically to the sexual activity with pleasure or in cases where they identified positive aspects of abuse such as affection or attention. ${ }^{36,37}$

\subsubsection{Injuries Sustained by Victims of Sexual Violence}

Various population-based studies of the characteristics of male sexual assault did not examine in detail the type of injuries sustained by male victims. Several studies using clinical samples have attempted to describe the characteristics of physical injuries sustained by male sexual assault victims, and their findings have been inconsistent. Some studies using small clinical samples have suggested that male victims, in comparison with female victims, are more likely to sustain severe physical injuries. ${ }^{8,30,38}$ Monroe et al. (2005) found that $41 \%$ of all participants in their study reported physical injuries sustained during the sexual assault. ${ }^{38}$ Stermac et al. (2004) reported one-third of all male victims presented to emergency care centers seeking treatment for severe physical injuries. ${ }^{8}$ Importantly, the severity of these injuries did not vary between males who were victimized by male or female perpetrators. Another comparative study of male and 
female victims seen at a rape-crisis center found that more women $(58 \%)$ than men $(40 \%)$ reported being physically harmed during sexual assault. ${ }^{39}$

\subsubsection{Psychological Consequences of Sexual Violence}

Sexual violence victimization has been associated with both long- and short-term poor mental health outcomes in both males and females. However, a majority of studies reporting on the prevalence of male sexual assault and associated adverse health outcomes have obtained their samples from mental health or trauma clinics and less is known about male victims who do not seek services. $^{7,28,40}$ A recent review of literature by Tewksbury $(2007)^{12}$ reported that men who were sexually victimized were more likely to report psychological problems when compared with non-victimized males. Also, males who were victimized as children were more likely to report mental health problems when compared with adult victims. ${ }^{41}$ There are few studies that have evaluated the gender differences in mental health outcomes of sexual assault. However, the emotions (e.g., experiences of fear, humiliation, and anger at the time of incident) associated with assault among males and females appear similar. ${ }^{20}$ Males who were raped as adults are also likely to experience post-traumatic stress disorder, depression, anxiety, anger, self-blame, and emotional distancing. ${ }^{7,21,42-44}$

Severe depression is a common problem reported by sexually assaulted males and females. ${ }^{7,15,45,}$ 46 Some studies have reported that male victims are more likely than female victims to present with severe depression. ${ }^{39,47}$ Research by Rentoul and Appleboom (1997) also showed that male victims reported high levels of anxiety. ${ }^{6}$ In addition to depression and social isolation, increased anger and hostility were observed among male victims who were treated immediately or years later. $^{39,42}$ Depression is often associated with suicide ideation/attempts and hostility among victimized males. ${ }^{7,18,48}$ A recent review of literature on male sexual assault indicated that 
suicide attempts are more common among adolescent and young adult victims. ${ }^{12}$ Juon and Ensminger (1997) reported that adverse life events such as sexual abuse are risk factors commonly associated with youth suicide. ${ }^{49}$ However, identifying the nature of the relationships between suicidal thoughts and traumatic experiences is made difficult by the underreporting of potentially stigmatizing experiences and the prevalence of suicidal thoughts among adolescents and young adults. A study of college-attending male victims of sexual assault indicated that $46 \%$ of all victims reported suicide ideation, $35 \%$ attempted suicide post-assault, $55 \%$ reported increased anger or rage, and 63.6\% reported increased interpersonal problems suggesting a possible relationship between sexual violence victimization and suicidal behavior. ${ }^{15}$ A study by Waldrop et al. (2007) investigating the interrelationships among victimization, PTSD, major depressive disorder, substance abuse, and suicidal behavior, reported that young adults with a history of sexual violence victimization were more likely to report suicide ideation and suicide attempts. ${ }^{50}$ Findings from a cross-sectional survey of adult males and females (16 years or older) in the general population indicated that exposure to sexual abuse was significantly related to suicidal behaviors. ${ }^{51}$ Though the relationships between sexual violence victimization and depression, ${ }^{52,53}$ depression and suicide ideation/attempt, ${ }^{54,55}$ and sexual assault and suicidal tendencies $^{56-58}$ are well-established among females, the relationships between sexual assault and suicide ideation among victimized males needs further investigation.

Suicide ideation is also considered an important correlate of deliberate self-harm (DSH) among both males and females. ${ }^{59,60}$ Some factors that have been linked with repetitive DSH among young adults include psychological distress and depressive symptomology which may include major depression and co-morbid suicide ideation. ${ }^{61-63}$ In a recent review of literature by Mangnall et al. (2008) sexual trauma (mainly childhood sexual trauma) was identified as the 
predisposing factor for DSH. ${ }^{63}$ Campbell et al. (2007) found considerably higher prevalence of DSH and mental health problems among victimized females. ${ }^{46}$ However, none of the studies investigating psychological consequences of sexual assault among adult males examined the prevalence or association of DSH with sexual assault.

\subsubsection{Externalized Behaviors among Male Victims}

Findings from previous studies suggest that the coping strategies of victimized males may be different from females. The general strain theory (GST) posits that certain strains or emotional stressors will increase the likelihood of aggressive behavior. ${ }^{64}$ Victimized males are known to take a express a greater number of externalizing behaviors and are less likely to display emotional expressions than female victims who may be more likely exhibit more internalizing emotions such as crying and restlessness. ${ }^{6}$ Victimized males commonly blame themselves and report more hostility and anger when compared to female victims. ${ }^{6}$ Other studies indicate that the long-term impact of sexual assault on males may be associated with an increased prevalence of expressions of anger, hostility, and aggressiveness, which have been identified with an increased risk for suicidal or violent behaviors. ${ }^{7,20}$ Studies have confirmed an association between sexual assault and violent behavior among victims. ${ }^{65-67}$ The GST may also explain gender differences in behavioral outcomes by suggesting that boys respond to strain with anger (an externalizing emotion) whereas girls have a greater tendency to respond to strain with depressed mood (an internalizing emotion). ${ }^{64,68}$ Sigfusdottir et al. (2008) examined the mediating effect of depression and anger in the relationship between sexual abuse and suicidal behavior and delinquency. ${ }^{68}$ Their findings suggested that anger resulting from sexual abuse was more strongly and positively related to outwardly directed forms of delinquency such as use of physical violence among young males than females. These findings underscore the future 
research to investigate the relationship between history of sexual assault and violent behavior among males.

\subsection{Current Gap in the Literature}

Studies that focused on adult male sexual assault estimated that prevalence of sexual assault of men in the community range from $0.6 \%$ to $22 \%$ depending on the demographic characteristics and sample population. ${ }^{5,15,69}$ Establishing incident characteristics and prevalence rates of male sexual assault is often challenging due to the lack of a proper data source, differing definitions of the sexual assault, and underreporting. Previous studies have primarily estimated the prevalence of sexual violence among males using data obtained from nationally administered surveys or using clinically-based samples. Most of the findings, though different from females, were not statistically significant; a finding that may be associated with small sample size. In addition to these data from clinical samples and population surveys, there remains a need to examine other data sources for a more complete understanding of various aspects of male sexual assault. Population-based surveys, such as BRFSS, provide information on the prevalence of male sexual assault in the general population. On the other hand, clinical studies capture a very small sample of male victims with adverse psychological problems. Because they rely less on victim-initiated disclosure in response to survey queries, criminological databases, such as the National Incident Based Reporting System (NIBRS), may capture details about large numbers of sexual assault victims and provide information on the characteristics of both perpetrators and the incident.

The study described in Chapter 2 examines the characteristics of male sexual assaults from 2001 to 2005 using NIBRS data. Incident-based reporting data was used to generate an epidemiological profile of male sexual assault where victim-, offender-, arrestee- and incidentrelated characteristics were analyzed. Findings from analysis of NIBRS data may complement 
current understanding about male sexual assault in the general population that has been derived from other data sources.

These adverse psychological consequences of sexual violence victimization can range from depression to suicidal behavior, and co-morbid diagnoses may be common. ${ }^{54,55,64}$ Previous studies suggest that female victims are more likely to express internalized emotions (e.g. depression, deliberate self-harm, etc.). ${ }^{46,70}$ and males are more likely to express externalized behavior (violence-related behavior). ${ }^{37,68}$ However, the associations between sexual violence victimization and externalizing behaviors among males are not well understood. To address this gap in the existing literature, we test several hypotheses related to the associations among mental health, violent behaviors, and sexual violence victimization among males.

Chapter 3 extends our understanding of adverse mental health consequences associated with male sexual assault victimization, which, in turn, will help to inform the development of prevention and intervention programs. The main objectives of this study were to explore gender differences in multiple categories of sexual violence victimization and the associations between victimization and adverse mental health outcomes among college age students attending a large U.S. university. A self-administered questionnaire was used to address various physical and psychological measures derived from standardized items and pre-tested surveys.

Another important aspect of sexual assault is how it is defined. Existing studies measuring the prevalence of sexual assault or examining associated adverse health outcomes among those who have been victimized have been limited to the differences in definitions of sexual assault. Among females, the most commonly used definition of sexual victimization includes rape or forced sexual intercourse. However, existing research has not provided a clear understanding of male 
sexual victimization. Existing studies have also demonstrated a strong relationship between sexual violence victimization and negative mental and physical health outcomes. ${ }^{29,71,72}$ Results from our earlier studies has shown that among males the strength of the associations between poor health status and sexual violence victimization is related to the severity (i.e. types) of victimization. ${ }^{29}$ Therefore, it is important to understand how males and females define sexual violence victimization. The objective of Chapter 4 is to identify the similarities and differences in the definition of sexual assault by young males and females.

In summary, there has been very little research on male sexual assault, and a poor understanding of the impact of sexual assault on the physical and mental health of male victims. There is also a paucity of research that explores the impact of sexual assault on violence-related behaviors among adult males. Further, there is little in-depth information about risk factors associated with sexual violence victimization among college-age males. This study will examine the relationship between sexual violence victimization and externalized behavior among young adult males in the general college population. Results of this study will advance scientific knowledge not only by increasing the understanding of the risk factors associated with adult male sexual assault, but will also help to develop an understanding of the gender-based differences in mental health outcomes among male and female victims of sexual assault. 
Chapter 2 


\section{Chapter 2}

\subsection{Introduction}

Sexual violence is a significant public health problem affecting both males and females. The majority of previous studies have evaluated various aspects of sexual violence victimization among females alone and few have provided data that could be used to estimate the rate of sexual assault among males. To date, the prevalence and characteristics of male sexual assault have been estimated using data obtained primarily through surveys of the general population (e.g. national surveys, community surveys, and university/college surveys) or through the use of smaller clinical samples.

Findings from the National Violence Against Women Survey (NVAWS) suggest that sexual violence victimization affects approximately 3 million men each year. ${ }^{4}$ Lifetime prevalence estimates from a national telephone survey conducted from 2001-2003 estimated that as many as 2.1 million men in the United States were victims of forced sex in their lifetime and 978,000 may have experienced unwanted sexual activity in the 12 months preceding the survey. ${ }^{11}$ In 2007, the National Crime Victimization Survey (NCVS) reported 11,300 rapes and sexual assault against males ages 12 years and over. ${ }^{73}$ Also, the rate of sexual assault among white males ( 1 per 1,000 persons ages 12 and older) was higher when compared with other races, and the rate was also higher among non-Hispanic males (1.1 per 1,000 males ages 12 and older) when compared with males of Hispanic origin. $^{73}$

Other studies have estimated the prevalence of male sexual violence to vary between 0.6$22.2 \%$; depending on the type of assault and population investigated. ${ }^{12,74}$ Stermac et al. (1996) reported that approximately $7 \%$ of males in the general U.S. population 
experienced some form of sexual violence. ${ }^{14}$ However, the true prevalence of sexual assault among males may be much higher. When compared with female victims, male victims are less likely to report a sexual assault. ${ }^{34,75,76}$ A review of literature by Rentoul and Appleboom (1997) also indicated that among all victims of rape who seek medical

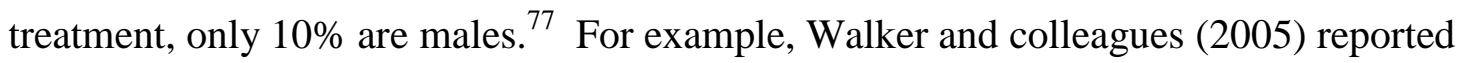
that more than $50 \%$ of male victims in their sample did not disclose their victimization for at least a year, and approximately $12 \%$ never disclosed it to anyone. ${ }^{7}$

Our current understanding of the characteristics of male sexual assault is primarily based on studies that have investigated the prevalence and consequences of childhood sexual abuse of boys. These studies have suggested that younger males (less than 11 years of age) who were non-white and of non-Hispanic origin were at greater risk for sexual assault. $^{78,79}$ Coxell and colleagues (1999) estimated the lifetime prevalence of nonconsensual sexual experience among males, and their results suggest that $2.89 \%$ of males experienced non-consensual sex as adults whereas $5.35 \%$ reported non-consensual sexual experiences as children. ${ }^{74}$ The NVAWS results indicated that $71 \%$ of male victims were raped before the age of 18 years. ${ }^{4}$ Basile and colleagues (2007) reported that among victimized men, the most frequently reported age of their first forced sexual experience was under the age of $12(41.3 \%, n=37)$, followed by the age-group, 12 to 17 years $(27.9 \%, \mathrm{n}=33) .^{11}$

For both males and females, the perpetrator was usually known to the victim. However, comparatively few existing studies have simultaneously examined the perpetrator and incident characteristics of male sexual assault. Findings from the NVAWS reported that most male victims (approximately 40\%) were sexually assaulted by acquaintances, such 
as friends, teachers, co-workers, or neighbors, and $22.8 \%$ of all male victims were assaulted by a stranger. ${ }^{4}$ The results of this study also suggested that the majority of the perpetrators were males. According to the NCVS 2007 survey, 58\% of rapes and sexual assault were committed by a friend or stranger. ${ }^{73}$ When compared with adults, adolescent victims of sexual assault are more likely to be perpetrated by an acquaintance or a relative. ${ }^{80-82}$ Similarly, Basile et al. (2007) reported that for male victims, $32.3 \%$ reported acquaintances, $17.7 \%$ reported non-intimate family members, $17.6 \%$ reported friends, and $15.9 \%$ reported intimate partners as the perpetrators of their first forced sex. ${ }^{2} \mathrm{~A}$ review of literature by Kaplan et al. (2001) suggested that $90 \%$ of sexual assault of males was perpetrated by males. ${ }^{75}$ Male perpetrators of male sexual assault more commonly reported themselves as heterosexuals, and in incidents where more than one assailant was involved, a perpetrator was more likely to use a weapon. ${ }^{82,83}$

Existing studies reporting of the characteristics of male sexual assault have not examined in detail the types of injuries sustained by victims. A few clinically-based studies have attempted to describe the characteristics of physical injuries sustained by male sexual assault victims, with their findings varying by sample. Some of these studies suggested that male victims, in comparison to female victims, are more likely to sustain severe physical injuries. ${ }^{8,30}$ Monroe et al. (2005) found that $41 \%$ of all participants in their sample $(n=125)$ reported physical injuries during sexual assault. ${ }^{38}$ Stermac et al. (2004) reported one-third of all male victims who presented to emergency care centers sought treatment for severe physical injuries such as lacerations, fractures, and internal injuries. ${ }^{8}$

Use of alcohol or other drugs are commonly associated with sexual assault incidents, especially among adolescents and young adults. ${ }^{84-86}$ Few studies to date have examined 
the involvement of alcohol in a sexual assault incident in which the victim was an adult male. Findings from a nationally representative study of U.S. adults suggested that $38 \%$ of male (versus $19.8 \%$ of female victims) victims and $58 \%$ of perpetrators of male sexual assault (versus $66.6 \%$ of perpetrators of female sexual assault) used alcohol at the time of incident. ${ }^{4}$ Isely and Gehrenbeck-Shim (1997) in their study of adult male victims of sexual assault, reported that $39 \%$ of males in their sample $(n=1,903)$ were assaulted while intoxicated. ${ }^{15}$ Another smaller study examining the characteristics of male victims $(n=29)$ of sexual assault who presented at a university sexual assault crisis unit found that $46 \%$ of all victims used alcohol, whereas $18 \%$ reported use of drugs. ${ }^{14}$ The authors of this study also noted that in $39 \%$ of the cases, the perpetrator also used alcohol.

Previous studies have estimated the prevalence of sexual violence among males using data obtained from nationally-administered surveys or using clinically-based samples. Most of the findings, though different from females, were not statistically significant due to low or small sample. In addition to these data sources, there remains a need to examine others for a more complete understanding of various aspects of male sexual assault. Population-based surveys, such as BRFSS, provide information on the prevalence of male sexual assault in the general population. On the other hand, clinical studies capture a very small sample of male victims with adverse psychological problems. Because they rely less on victim-initiated disclosure in response to survey queries, criminological databases, such as NIBRS, may capture details about large numbers of sexual assault victims and provide information on the characteristics of both perpetrators and the incident. Findings from analysis of NIBRS data may complement current understanding about male sexual assault in the general population that have been 
derived from population-based surveys and clinical samples. The objective of this study was to examine the characteristics of male sexual assaults from 2001 to 2005 using NIBRS data. The incident-based reporting system data was used to generate an epidemiological profile of male sexual assault where victim-, offender-, arrestee- and incident-related characteristics will be examined.

\subsection{Methods}

\subsubsection{Data}

The data from 2001 to 2005 extract files of NIBRS were used in order to examine the victim, offender, and incident characteristics of male sexual assault. NIBRS is an incident-based reporting system and therefore collects information on every single crime occurrence in the 29 participating states that were reported to the police. The five-year NIBRS data was obtained from the Inter-University Consortium for Political and Social Research (ICPSR) at the University of Michigan. The extract files of NIBRS data contain data that are merged, restructured, and organized into four major files. These four files are organized based on four units of analysis: incident, victim, offender, and arrestee. Each single file contains information on related NIBRS segments (record type). Detailed information on 2001 to 2005 extract file organization and contents can be obtained from the NIBRS data codebooks for each year. ${ }^{87}$ For the current study, all four segments of levels were used. The six forcible and non-forcible sexual assault offenses committed against male victims used in this study included: forcible rape, forcible sodomy, sexual assault with an object, forcible fondling, incest, and statutory rape. Therefore, the definition of sexual assault among males in this study is based on these six unwanted sexual experiences (forcible and non-forcible). Available data included 
detailed information on incident, offender, victim, and arrestee characteristics. There were 29 states participating in the NIBRS program through 2004. There were five states where NIBRS was relatively new and covered only a small percentage of their populations. Therefore, data from 24 states (AR, CO, CT, DE, ID, IA, KS, MA, MI, MT, NE, NH, ND, OH, OR, RI, SC, SD, TN, UT, VT, VA, WV, WI) where NIBRS covered more than $15 \%$ of the state population over the five-year study period, were included in this study. The rates of male sexual assault were calculated using 2000 census data for all 24 states. $^{88}$

\subsubsection{Measures}

In order to create an epidemiological profile of male sexual assault, victim-, offender-, arrestee-, and incident-related characteristics were examined. Only those incidents where the first reported offense was forcible (forcible rape, forcible sodomy, sexual assault with an object, forcible fondling) or non-forcible (incest and statutory rape), sexual assault was included in the analyses. Variables assessing the incident-related characteristics included the type of sexual assault (forcible, non-forcible), location (public place, residence, school/college, jail, outdoors), type of weapon used (firearm/sharp object, personal force, drugs/narcotics, other, none), and time of incident occurrence (incident date/hour). The victim-related characteristics (unit of analysis: victim) included information specific to the victim (age, race, ethnicity, offender-victim relationship). The variables assessing these characteristics included race (White, African-American, American Indian/Alaskan Native, Asian/Pacific Islander), ethnicity (Hispanic, Non-Hispanic), relationship with the offender (within family, outside family but known to the victim, not known by victim), and type of injury sustained by the victim (none, minor injury, major injury, any injury). 
The age of the victim was categorized into nine groups $(0-9,10-19,20-29,30-39,40-49$, $50-59,60-69,70-79,80+)$ based on the availability of Census data. For consistency, the age of offender and arrestee were also categorized in the same groups. The variables assessing offender-related information (unit of analysis: offender) included race, gender, age, and use of alcohol or other substance by the offender. The variables assessing arrestee-related (unit of analysis: arrestee) information include race, gender, and age.

\subsubsection{Statistical Analysis}

Since not all 24 states had $100 \%$ participation in the NIBRS program, population data based on the percentage of population covered by NIBRS in an individual state were used for each state included in the analysis. For example, NIBRS covered $78 \%$ of the population in Arkansas; therefore $78 \%$ of the total male population was used in the denominator. In order to lower any bias in male population size calculation, the percentage of males and females in each county was examined for all states in this study. Race-specific rates were calculated for only White and African-American males due to small sample sizes of other racial groups. Age- and race-specific rates of sexual assault per 100,000 persons, 95\% confidence intervals (CI), and rate ratios were calculated. Rate ratios were calculated using 2001 as the reference year.

The logistic regression model was used for the analysis. Since individual-level binary outcomes were aggregated over each of the age groups, the logistic model was fit to the aggregated response (i.e., Total \# of events within Male /Total Male Population), which was binomially distributed as opposed to Bernoulli distribution at the individual subject level. 
The model proposes for each observation there is a set of explanatory variables (i.e. time and age, along with the interaction of time with age) that might inform the final probability. The logits (or logarithm of the odds) of the unknown binomial probabilities are modeled as a linear function of the explanatory variables. For example, for the analysis involving the Male study group, $\mathrm{p}_{\mathrm{i}}(\mathrm{i}=1, \ldots, 45)$ in this case is the respective observation for the Total \# events with Males /Total Male Population and the logistic model fit has the following form:

$\log i t\left(p_{i}\right)=\log \left(\frac{p_{i}}{1-p_{i}}\right)=\alpha+\beta_{1} \times \operatorname{Age}_{i}+\beta_{2} \times$ Time $_{i}+\beta_{3} \times$ Age $_{i} \times$ Time $_{i}$

Where $\alpha=$ intercept for the model, and $\beta_{\mathrm{i}}, \mathrm{i}=1,2,3$ =estimate for the respective covariate.

The test used to assess the significance of the parameter for each covariate (Age, Time and Age*Time Interaction) is the likelihood ratio test. This test has an asymptotic chisquare distribution under the hypothesis that the underlying parameter to be tested is zero. Thus, these statistics can be used in a test of hypothesis of the significance of each additional term fit. ${ }^{89}$ The confidence intervals on the output are the Wald Confidence Intervals, defined as: $\hat{\beta} \pm z_{1-\alpha / 2^{\widehat{\sigma}}}$, where $z_{p}$ is the $100 p$ th percentile of the standard normal distribution, $\hat{\beta}$ is the parameter estimate, and $\hat{\sigma}$ is the estimate of its standard error.

The likelihood ratio test was also used to test each time trend separately (since only one time was used for each of these models, these models simplify to one covariate for Age), for the following hypothesis:

$\mathrm{H}_{0}$ : Time does not change the log odds of the response 
$\mathrm{H}_{1}$ : Time changes the log odds of the response

All analyses were performed using Microsoft Excel and statistical computer software SAS (Ver. 9.1). ${ }^{90}$

\subsection{Results}

From 2001 to 2005 there was an average of 6,714 incidents of male sexual assault reported each year to NIBRS. For the 5-year period, the average number of victims and offenders of male sexual assault were approximately 8,181 and 7,407, respectively. On average, more than 1,460 arrests were made each year for male sexual assault from 2001 to 2005 . There was an average of 1.2 victims and 1.1 offenders per incident.

Demographic characteristics of male sexual assault victims and age-, race-, and ethnicityspecific rates of sexual assault among males are shown in Table 2.1. The majority of the victims were under the age of 19 for all five years (approximately $80 \%$ each year) and White (range: $80.02 \%-83.90 \%$ ). Data on ethnicity was missing for about $40 \%$ of victims; however, when information was available, most were not of Hispanic origin. Upon examining the relationship of the victim to the offender (Table 2.1), the majority of the offenders were known to the victim as a family member (2001: 38.84\%; 2002: 39.49\%; 2003:40.75\%; 2004: $39.07 \% ; 2005: 39.19)$ or someone known to the victim but not a family member (2001: 56.09\%; 2002: 55.92\%; 2003: 55.28\%; 2004: 56.36\%; 2005: $55.04 \%)$. Overall, a very small percentage of males were victimized by a stranger (3.96\% to $5.41 \%)$.

The rates of male sexual assault are described in Table 2.2. Over the 5-year period, the rate of sexual assault was highest among boys between the ages of 1 to 9 years, followed by boys between the ages of 10 to 19 years. For both groups, the highest rates were 
observed in 2005, i.e., 95.81 per 100,000 persons (95\% CI $89.74-101.87$ ) for boys ages 19 years and 69.02 per 100,000 person (95\% CI 63.87-74.17) for boys ages 10-19 years. The lowest rate of sexual assault was observed among males above the age of 50 years; however, these rates may be unstable due to small sample sizes. In each year, the rate consistently decreased in all age groups through age 79 and then increased in the oldest group. Using year 2001 as the reference year, rate ratios for all age groups were calculated for the remaining years (see Table 2.2). The overall rates of male sexual violence were $10 \%$ higher in 2002 and 2003, 25\% higher in 2004, and 35\% higher in 2005 as compared to 2001. From 2001 through 2004, rate increases were observed in most age groups. However, for males who were between the ages of 60-69 years, the rate decreased nearly $50 \%$ in 2003 as compared to 2001 . For males who were 80 years old or above the rate decreased $25 \%$ in 2002 and $28 \%$ in 2003 . The rate then increased $10 \%$ in 2001 and $30 \%$ in 2005 as compared to 2001.

Race- and age-specific rates of male sexual assault from 2001 to 2005 are shown in Table 2.2. As reported earlier, the rate was higher among younger males (0-9 years, and 10-19 years), decreasing with age in both races. The rate was higher among African-American males for all age groups as compared to White males. The rate was higher among African-American males for all five years and highest among this group in 2005 (49.69 per 100,000 persons, $95 \%$ CI 45.32-54.06). However, the rate increased slightly for both White and African-American males who were 80 or older. Among White males, the rate of sexual assault steadily increased since 2001 for the majority of the age groups. In 2005 , the rate increased by $248 \%$ for White males who were between the ages of $70-79$ years. For the oldest age group (80+), the rate decreased through 2003 and then 
increased by $14 \%$ in 2004 and 2005. For African-American males, the rate increased among males who were between the ages of 0-39 years from 2001 to 2005 . The rates of sexual assault decreased by $80 \%$ for males who were between the ages of $60-69$ years during years 2003 and 2005. For the oldest age group (80+) the rate of sexual assault increased by $197 \%$ for African- American males in the year 2005 .

These findings were further supported by the time-trend analysis (see Table 2.3). All covariates (age, time, and age by time) were significant for the Chi-Square test (p-value $<0.0001)$. Interaction of age by time was significant ( $\mathrm{p}$-value $<0.0001)$ indicating age groups have different time trends. We then examined each group for time trends separately. A significant change over time was observed for age groups 0-9, 10-19, 2029, 30-39, 40-49, 50-59, and 70-79. We also examined the time trends for White and African-American males by age group over the 5-year study period (Tables 2.4A and 2.4B). The time trend analyses for White males were similar to those observed among all males. However, we did not observe any age group by time interaction for the AfricanAmerican males ( $\mathrm{p}$-value $=0.7399)$. When the individual age groups were examined separately, significant time trend differences were observed only for young AfricanAmerican males (age group 0-9: p-value <0.0001; age group 10-19: p-value <0.001; age group 10-29: p-value <0.0001).

The rate of male sexual assault based on the ethnicity of the victims was also calculated (see Table 2.2). The rate was higher among Hispanic males compared to non-Hispanic males across all years. The rate of sexual assault decreases over the five year study period for both Hispanic and non-Hispanic males. However, when comparing these rates 
it is important to consider they may be biased, as data on ethnicity was not reported on approximately $38-42 \%$ of the victims.

Table 2.5 reports characteristics of male sexual assault offender and arrestee as reported by victims. The majority of the offenders were male (2001: 83.05\%; 2002: 83.29\%; 2003: 83.40\%; 2004: 83.68; 2005: 83.00\%); White (2001: 80.72\%; 2002: 81.93\%; 2003: 80.18; 2004: 79.83\%; 2005: 77.04) and were under the age of 39. Demographic characteristics of arrestees of male sexual assault are also shown in Table 2.2. Over the 5-year period, an average of $<1$ arrest per incident of male sexual assault was made. Most of the arrestees were male (range: $85.89 \%$ to $87.84 \%$ ), White (range: $79.99 \%$ to 85.23\%), and between the ages of 10-19 (2001: 52.23\%; 2002: 45.15\%; 2003: 46.92\%; 2004: 50.15\%; 2005:49.18\%). The annual averages of all sexual offenses by offender and arrestee gender over the 5-year period are shown in Figure 2.1. The majority of the forcible sexual assaults (forcible sodomy: 94.99\%; sexual assault with an object: $85.29 \%$; forcible fondling: $85.62 \%$ ) were perpetrated by males, whereas a large number of forcible rapes (73.2\%) were perpetrated by females (Figure 2.1A). A similar pattern was also observed for arrestees (Figure 2.1B).

The male sexual assault incident characteristics are reported in Table 2.6. Forcible sexual assaults, which include rape, sodomy, sexual assault with an object, and forcible fondling, were the highest reported incidents of male sexual assault. Of these, the highest reported incidents of male sexual assault were forcible fondling (2001: 52.84\%; 2002: 53.79\%; 2003: 54.44\%; 2004: 53.78\%; 2005: 53.42\%) and forcible sodomy (2001: 33.20; 2002: $31.89 ; 2003: 31.07 \% ; 2004: 30.88 \% ; 2005: 30.77 \%)$. The majority of these sexual assault incidents took place at a residential location $(2001: 74.69 \% ; 2002: 74.85 \%$; 2003 : 
76.37\%; 2004: 73.59\%; 2005: 73.37\%) and did not involve use of a weapon, as the majority of the incidents indicated that the offender used personal force against the victim (2001: 62.30\%; 2002: 62.53\%; 2003: 61.94\%; 2004: 60.07\%; 2005: 61.37\%).

The nature of injuries sustained by male victims of sexual assault was also analyzed. Table 2.7 shows the average annual age-specific rates of injuries sustained by male victims of sexual assault. Results indicate that for any specific age group, the majority of the victims did not receive any injuries. The age-specific trend of average annual injury rate sustained by males as a result of a sexual assault is shown in Figure 2.2.

The time of occurrence of male sexual assault incidents was also examined. Figure 2.3 shows the trend of incident hour for male sexual assaults from 2001 to 2005; the majority of the incidents occurred either at midnight or during the day between 8 am and $2 \mathrm{pm}$. Similar trends were observed each year.

\subsection{Discussion}

This study provides a five-year epidemiological profile of male sexual assault using NIBRS data. To our knowledge, this is the first study to estimate age-, race-, and ethnicity-specific rates of sexual assault among males and examine the victim, offender, arrestee, and incident characteristics using an incident-based reporting system. NIBRS captures a wide range of information on offense, victim, offender, and arrestee characteristics on offenses brought to the attention of law enforcement. ${ }^{91}$ This study substantiates, concurrent with extant research, that males report sexual assault throughout their lifetime. $^{74,76,78,92}$ However, a large number of sexual assault incidents involved males under the age of 29. The prevalence and characteristics of sexual violence among males have primarily been estimated using data obtained from nationally-administered 
surveys or using clinically-based samples. Most of the findings, though different from females, may have been limited in their ability to detect significant differences due to low sample size. In addition to these data sources, there is an urgent need to examine other data sources such as criminological data to provide a more complete understanding of male sexual assault. Findings from analysis of NIBRS data addresses this need by complementing current data systems and increasing understanding of the extent of male sexual assault in the general population.

Overall, rates from 2001 to 2005 for all males were comparable to the rates reported by NCVS for these same years. However, observed rates were more stable as the actual number of incidents that were reported to the police were used. ${ }^{93-97}$ It is likely that not all incidents of male sexual assault are reported to law enforcement. The lower rate of sexual assault among older age groups (>30 years) may be due the under-reporting of sexual assault by adult males. Previous research has suggested that older males are less likely to report sexual assault to law enforcement, especially when the assault is more severe such as in the case of rape. ${ }^{4,38,98}$ Tjaden and Theoness (2006) reported that only $12.9 \%$ of the male victims who were raped after their $18^{\text {th }}$ birthday reported their rape to the police. ${ }^{4}$ This finding is consistent with a growing body of evidence that suggests that under-reporting among adult males is function of social beliefs about vulnerability and masculinity, reporting patterns associated with sexual orientation, and age at time of victimization. $^{5,34,77,99,100}$ Additionally, not all 50 states participate in the NIBRS program and therefore our estimated rates of sexual assault among males may not be representative of the larger U.S. adult male population. 
Our results showed the highest rates of sexual assault were observed among the two youngest age groups (0-9 years and 10-19 years), a pattern commonly reported in previous studies. $5,15,79,101,102$ Our results also showed that rates of sexual assault were higher among African-American males between the ages of 0-9 and 10-19 years. However, we were unable to find a direct age- and race-specific rate comparison of sexual assault as the majority of the existing literature provides information on prevalence of sexual assault among children. ${ }^{74,78,103}$ A review of literature by Holmes et al. (1998) reported that studies focused on child sexual abuse reporting a higher prevalence of sexual abuse among non-white boys, especially African-American boys. ${ }^{79}$ Other studies using survey data have also reported a higher prevalence of sexual assault among African-American boys when compared to White boys who were under the age of $12 .^{79,81}$ We also observed an increasing trend in the rate of sexual assault for the youngest age groups (0-9 years and 10-19 years). A similar trend was observed for the White and African-American boys in these age groups. This increase in rate among younger groups may due to increased reporting of the incidents.

Overall, the rate ratios showed that the age- and race-specific rates of sexual assault consistently increased over the years. These increased rates, especially among younger males, indicate the severity of this problem in the male population. Various studies suggest that males who experience sexual assault as children are at greater risk of negative mental health outcomes, ${ }^{104,105}$ suicidal behavior, ${ }^{49,}$ 67, 106 and risk behaviors such as alcohol and drug abuse. ${ }^{107-109}$ Also of concern are elderly males 80 years and older whose rates were shown to increase. Few studies in the past have examined the prevalence of sexual assault experienced by elderly males. ${ }^{110-112}$ Similar to young males, 
these studies also indicated that sexual assault experiences lead to poor mental health outcomes among elderly males. ${ }^{112}$ Higher rate of sexual assault among younger and elderly males indicate need for more focused intervention programs for these two at-risk populations.

We also examined the demographic characteristics of the perpetrators. As reported in previous studies, our results confirm that most of the perpetrators of male sexual assault were males. $^{4,83,113}$ Our results parallel the findings from McCloskey et al. (2005), indicating that the majority of the perpetrators were younger (<39 years). ${ }^{114}$

Some previous studies have suggested that a male being sexually assaulted by a female may be difficult to conceptualize or measure in self-report surveys. ${ }^{8,115}$ Even though the majority of the perpetrators were males, a significant number of males were also victimized by women. Particularly, the majority of the offenses like forcible rape and incest, were committed by female perpetrators. There are a handful of studies that have reported females as perpetrators of male sexual assault, however type and severity of the assault were not studied. ${ }^{31,115-117}$ This study also provided information about the relationships between victim and offender of sexual assault. Similar to previous studies, our findings indicated that males are more likely to be victimized by a friend/acquaintance. ${ }^{4,75,82,113,118}$

The arrestee characteristics were similar to those of offenders; however, a relatively small number of arrests for male sexual assault were made each year. None of the previous studies about male victims of sexual assault examined the criminal prosecution of offenders or reported arrestee characteristics. Tjaden and Theoness (2006), when examining justice system outcomes of female sexual assault, reported that $37 \%$ of the 
rapes against women reported to the police resulted in the criminal prosecution of the rapist. ${ }^{4}$ However, existing studies have not examined the likelihood of arrest in a case of male sexual assault. Chilton and Jarvis (1999) reported that NIBRS data may provide more accurate arrest estimates, therefore suggesting an increased reliability of rates calculated using NIBRS data when examining offender and arrestee characteristics. ${ }^{119}$ Our findings, in concordance with those reported previously, suggest that the majority of the incidents reported by male victims were forcible sodomy and forcible fondling., 27 , 101, 120 The distinction between forcible rape and forcible sodomy is based on the definition used by NIBRS. The forcible rape is defined as "the carnal knowledge of a person, forcibly and/or against that person's will; or, not forcibly or against the person's will where the victim is incapable of giving consent because of his/her temporary or permanent mental or physical incapacity. If force was used or threatened, the crime should be classified as forcible rape regardless of the age of the victim. If no force was used or threatened and the victim was under the statutory age of consent, the crime should be classified as a Non-forcible Sex Offense (Statutory Rape). ${ }^{121}$ " Whereas forcible sodomy is defined as "Oral or anal sexual intercourse with another person, forcibly and/or against that person's will; or not forcibly or against the person's will where the victim is incapable of giving consent because of his/her youth or because of his/her temporary or permanent mental or physical incapacity. ${ }^{121}$ " These are distinctly different crimes for female victims, but very similar for male victims. This may also be the reason that a large percentage of female offenders committed forcible rape as compared to male offenders. Previous studies examining the type of sexual assault among males also indicated that relatively less severe forms of sexual assault, such as 
forcible touching, kissing, and other forms of sexual harassment, were more common among males as compared to females. ${ }^{85,122}$

Many studies, especially those focusing on college-attending populations, have suggested a strong relationship between alcohol, ${ }^{81,84-86,92,123,124}$ substance abuse, ${ }^{75,82}$ and sexual assault victimization. However, our results showed a very small number of incidents $(1.85-2.75 \%)$ of sexual assault where alcohol or other substances were used by the offender. The reasons for discrepancies are not clear, but this may be due to the underreporting of such incidents as victims, especially those who were underage, were also consuming alcohol or illegal substances. Studies using self-reported survey data have indicated that a significant amount of male victims were assaulted while intoxicated. ${ }^{15,85 \text {, }}$ 120

Various studies have also reported involvement of weapons in male sexual assault incidents. ${ }^{8,81,83}$ However, our findings suggest that no weapon (other than personal force) was used in the majority of the incidents. The majority of the reported incidents occurred near midnight or during the day, between 8 am and $2 \mathrm{pm}$. When examining the time of incident it is important to consider how time of incident is recorded by NIBRS. For example, if an incident occurred between midnight and 3 am, the record will show the time of incident as “ 00 ” (i.e. midnight). Similar findings were also reported by Snyder (2000) who examined sexual assault of children using NIBRS data. ${ }^{101}$ Furthermore, the most common location of sexual assault reported in NIBRS was a residential place. Previous studies examining the sexual assault of males in noninstitutionalized populations have reported similar findings. ${ }^{15,98,101}$ 
Our study provides a 5-year epidemiological profile of male sexual assault using NIBRS data; however, there are a few limitations that should be considered. First, only offenses reported to law enforcement agencies that voluntarily provided information to NIBRS were included. Therefore, only 24 states out of 50 were represented and not all law enforcement agencies in participating states provided data to NIBRS. Thus, these findings may not be an accurate account of the actual number of male sexual assault offenses, and the data may not be generalizable to the U.S. or non-participating states. Second, differences in definitions of sexual assault used by NIBRS and previous studies may limit the direct comparison of our findings to others, as survey-based research has typically used broader definitions of sexual assault. Third, when compared to surveybased studies, NIBRS data have larger numbers of incidents. However, it does not collect information on general health indicators, therefore limiting the use of NIBRS data to measure the total burden of sexual offenses committed against males.

In conclusion, the overarching goal of this study was to provide a more complete epidemiological profile of male sexual assault. The characteristics of victims, offenders, arrestees, and incidents of male sexual assault were addressed in great detail. In addition, detailed information about the type of injury sustained by victims, as well as a temporal (incident hour) profile of sexual assault incidents were also provided. The NIBRS data describe the rate of violent crimes in this country. However, since many crimes are not reported to law enforcement, police estimates of assaultive violence are typically lower than those obtained by social scientists conducting epidemiological research. ${ }^{73,125}$ Thus, these data may suggest a lower rate of male sexual assault than previously reported. Despite these limitations, findings from NIBRS data will complement the results from 
population-based studies since a majority of these studies uses relatively smaller sample sizes of male victims. 
Table 2.1: Characteristics of Male Sexual Assault Victims

\begin{tabular}{|c|c|c|c|c|c|}
\hline \multirow[t]{2}{*}{ Variables } & \multicolumn{5}{|c|}{$\begin{array}{c}N \\
\text { (Percentage) }\end{array}$} \\
\hline & 2001 & 2002 & 2003 & 2004 & 2005 \\
\hline \multicolumn{6}{|l|}{ Age Groups (in years) } \\
\hline All & $\begin{array}{l}6129 \\
(100)\end{array}$ & $\begin{array}{l}7000 \\
(100)\end{array}$ & $\begin{array}{r}7248 \\
(100)\end{array}$ & $\begin{array}{l}8456 \\
(100)\end{array}$ & $\begin{array}{l}9150 \\
(100)\end{array}$ \\
\hline $0-09$ & $\begin{array}{c}3206 \\
(52.31)\end{array}$ & $\begin{array}{c}3504 \\
(50.06)\end{array}$ & $\begin{array}{c}3794 \\
(52.35)\end{array}$ & $\begin{array}{c}4219 \\
(49.89)\end{array}$ & $\begin{array}{c}4464 \\
(48.79)\end{array}$ \\
\hline $10-19$ & $\begin{array}{c}2282 \\
(37.23)\end{array}$ & $\begin{array}{c}2668 \\
(38.11)\end{array}$ & $\begin{array}{c}2598 \\
(35.84)\end{array}$ & $\begin{array}{c}3148 \\
(37.23)\end{array}$ & $\begin{array}{c}3383 \\
(36.97)\end{array}$ \\
\hline $20-29$ & $\begin{array}{c}277 \\
(4.52)\end{array}$ & $\begin{array}{c}394 \\
(5.63)\end{array}$ & $\begin{array}{c}421 \\
(5.81)\end{array}$ & $\begin{array}{c}517 \\
(6.11)\end{array}$ & $\begin{array}{c}616 \\
(6.73)\end{array}$ \\
\hline $30-39$ & $\begin{array}{c}184 \\
(3.00)\end{array}$ & $\begin{array}{c}234 \\
(3.34)\end{array}$ & $\begin{array}{c}220 \\
(3.04)\end{array}$ & $\begin{array}{c}292 \\
(3.45)\end{array}$ & $\begin{array}{c}309 \\
(3.38)\end{array}$ \\
\hline $40-49$ & $\begin{array}{c}106 \\
(1.73)\end{array}$ & $\begin{array}{c}127 \\
(1.81)\end{array}$ & $\begin{array}{c}132 \\
(1.82)\end{array}$ & $\begin{array}{c}173 \\
(2.05)\end{array}$ & $\begin{array}{c}225 \\
(2.46)\end{array}$ \\
\hline $50-59$ & $\begin{array}{c}35 \\
(0.57)\end{array}$ & $\begin{array}{c}38 \\
(0.54)\end{array}$ & $\begin{array}{c}49 \\
(0.68)\end{array}$ & $\begin{array}{c}61 \\
(0.72)\end{array}$ & $\begin{array}{c}98 \\
(1.07)\end{array}$ \\
\hline $60-69$ & $\begin{array}{c}23 \\
(0.38)\end{array}$ & $\begin{array}{c}23 \\
(0.33)\end{array}$ & $\begin{array}{c}13 \\
(0.18)\end{array}$ & $\begin{array}{c}27 \\
(0.32)\end{array}$ & $\begin{array}{c}23 \\
(0.25)\end{array}$ \\
\hline $70-79$ & $\begin{array}{c}7 \\
(0.11)\end{array}$ & $\begin{array}{c}5 \\
(0.07)\end{array}$ & $\begin{array}{c}14 \\
(0.19)\end{array}$ & $\begin{array}{c}8 \\
(0.09)\end{array}$ & $\begin{array}{c}19 \\
(0.21)\end{array}$ \\
\hline $80+$ & $\begin{array}{c}9 \\
(0.15) \\
\end{array}$ & $\begin{array}{c}7 \\
(0.10) \\
\end{array}$ & $\begin{array}{c}7 \\
(0.10) \\
\end{array}$ & $\begin{array}{c}11 \\
(0.13) \\
\end{array}$ & $\begin{array}{c}13 \\
(0.14) \\
\end{array}$ \\
\hline \multicolumn{6}{|l|}{ Race } \\
\hline White & $\begin{array}{c}5142 \\
(83.90)\end{array}$ & $\begin{array}{c}5852 \\
(83.60)\end{array}$ & $\begin{array}{c}5996 \\
(82.73)\end{array}$ & $\begin{array}{c}6979 \\
(82.45)\end{array}$ & $\begin{array}{c}7322 \\
(80.02)\end{array}$ \\
\hline African- American & $\begin{array}{c}948 \\
(15.47)\end{array}$ & $\begin{array}{c}1097 \\
(15.67)\end{array}$ & $\begin{array}{c}1209 \\
(16.68)\end{array}$ & $\begin{array}{c}1441 \\
(17.02)\end{array}$ & $\begin{array}{c}1742 \\
(19.04)\end{array}$ \\
\hline Other & $\begin{array}{c}39 \\
(0.64)\end{array}$ & $\begin{array}{c}51 \\
(0.73)\end{array}$ & $\begin{array}{c}43 \\
(0.59)\end{array}$ & $\begin{array}{c}45 \\
(0.53)\end{array}$ & $\begin{array}{c}86 \\
(0.94) \\
\end{array}$ \\
\hline \multicolumn{6}{|l|}{ Ethnicity } \\
\hline Hispanic Origin & $\begin{array}{c}298 \\
(4.54)\end{array}$ & $\begin{array}{c}331 \\
(4.42)\end{array}$ & $\begin{array}{c}271 \\
(3.47)\end{array}$ & $\begin{array}{c}415 \\
(4.56)\end{array}$ & $\begin{array}{c}499 \\
(5.03)\end{array}$ \\
\hline Not of Hispanic Origin & $\begin{array}{c}3719 \\
(56.60)\end{array}$ & $\begin{array}{c}4061 \\
(54.20)\end{array}$ & $\begin{array}{c}4321 \\
(55.28)\end{array}$ & $\begin{array}{c}4946 \\
(54.36)\end{array}$ & $\begin{array}{c}5167 \\
(52.04)\end{array}$ \\
\hline Unknown & $\begin{array}{c}2554 \\
(38.87) \\
\end{array}$ & $\begin{array}{c}3101 \\
(41.39) \\
\end{array}$ & $\begin{array}{r}3225 \\
(41.26) \\
\end{array}$ & $\begin{array}{c}3738 \\
(41.08) \\
\end{array}$ & $\begin{array}{r}4262 \\
(42.93) \\
\end{array}$ \\
\hline \multicolumn{6}{|l|}{ Relationship to Offender } \\
\hline Within family & $\begin{array}{c}2174 \\
(38.84)\end{array}$ & $\begin{array}{c}2475 \\
(39.39)\end{array}$ & $\begin{array}{c}2673 \\
(40.75)\end{array}$ & $\begin{array}{c}3002 \\
(39.07)\end{array}$ & $\begin{array}{c}3261 \\
(39.19)\end{array}$ \\
\hline $\begin{array}{l}\text { Outside family but known to the } \\
\text { victim }\end{array}$ & $\begin{array}{c}3140 \\
(56.09)\end{array}$ & $\begin{array}{c}3505 \\
(55.92)\end{array}$ & $\begin{array}{c}3626 \\
(55.28)\end{array}$ & $\begin{array}{c}4331 \\
(56.36)\end{array}$ & $\begin{array}{c}4609 \\
(55.04)\end{array}$ \\
\hline Not known by victim & $\begin{array}{c}284 \\
(5.07)\end{array}$ & $\begin{array}{c}288 \\
(4.59) \\
\end{array}$ & $\begin{array}{c}260 \\
(3.96)\end{array}$ & $\begin{array}{c}351 \\
(4.57)\end{array}$ & $\begin{array}{c}450 \\
(5.41) \\
\end{array}$ \\
\hline
\end{tabular}


Table 2.2: Rate and Rate Ratios of Sexual Assault among Males by Age, Race, and Ethnicity from 2001 to 2005

\begin{tabular}{|c|c|c|c|c|c|c|c|c|c|c|}
\hline \multirow[t]{2}{*}{ Variables } & \multicolumn{5}{|c|}{$\begin{array}{l}\text { Sexual Assault Rate per 100,000 Males } \\
(95 \% \mathrm{CI})\end{array}$} & \multicolumn{5}{|c|}{ Rate Ratios } \\
\hline & 2001 & 2002 & 2003 & 2004 & 2005 & $2001^{\S}$ & 2002 & 2003 & 2004 & 2005 \\
\hline \multicolumn{11}{|l|}{ Age Groups (in years) } \\
\hline All & $\begin{array}{c}21.02 \\
(18.18,23.86)\end{array}$ & $\begin{array}{c}23.03 \\
(20.05,26.00)\end{array}$ & $\begin{array}{c}23.09 \\
(20.11,26.06)\end{array}$ & $\begin{array}{c}26.18 \\
(23.01,29.36)\end{array}$ & $\begin{array}{c}28.33 \\
(25.03,31.63)\end{array}$ & 1.00 & 1.10 & 1.10 & 1.25 & 1.35 \\
\hline $0-09$ & $\begin{array}{c}75.89 \\
(70.49,81.28)\end{array}$ & $\begin{array}{c}79.65 \\
(74.12,85.18)\end{array}$ & $\begin{array}{c}83.63 \\
(77.96,89.30)\end{array}$ & $\begin{array}{c}90.55 \\
(84.65,96.45)\end{array}$ & $\begin{array}{c}95.81 \\
(89.74,101.87)\end{array}$ & 1.00 & 1.05 & 1.10 & 1.19 & 1.26 \\
\hline $10-19$ & $\begin{array}{c}51.54 \\
(47.09,55.99)\end{array}$ & $\begin{array}{c}57.86 \\
(53.15,62.58)\end{array}$ & $\begin{array}{c}54.60 \\
(50.02,59.18)\end{array}$ & $\begin{array}{c}64.23 \\
(59.26,69.19)\end{array}$ & $\begin{array}{c}69.02 \\
(63.87,74.17)\end{array}$ & 1.00 & 1.12 & 1.06 & 1.25 & 1.34 \\
\hline $20-29$ & $\begin{array}{c}6.81 \\
(5.19,8.43)\end{array}$ & $\begin{array}{c}9.31 \\
(7.42,11.20)\end{array}$ & $\begin{array}{c}9.64 \\
(7.71,11.56)\end{array}$ & $\begin{array}{c}11.53 \\
(9.42,13.63)\end{array}$ & $\begin{array}{c}13.73 \\
(11.44,16.03)\end{array}$ & 1.00 & 1.37 & 1.42 & 1.69 & 2.02 \\
\hline $30-39$ & $\begin{array}{c}4.12 \\
(2.86,5.38)\end{array}$ & $\begin{array}{c}5.01 \\
(3.62,6.40)\end{array}$ & $\begin{array}{c}4.56 \\
(3.24,5.89)\end{array}$ & $\begin{array}{c}5.90 \\
(4.40,7.41)\end{array}$ & $\begin{array}{c}6.24 \\
(4.70,7.79)\end{array}$ & 1.00 & 1.22 & 1.11 & 1.43 & 1.52 \\
\hline $40-49$ & $\begin{array}{c}2.35 \\
(1.40,3.30)\end{array}$ & $\begin{array}{c}2.69 \\
(1.68,3.71)\end{array}$ & $\begin{array}{c}2.71 \\
(1.69,3.73)\end{array}$ & $\begin{array}{c}3.45 \\
(2.30,4.60)\end{array}$ & $\begin{array}{c}4.49 \\
(3.17,5.80)\end{array}$ & 1.00 & 1.15 & 1.15 & 1.47 & 1.91 \\
\hline $50-59$ & $\begin{array}{c}1.06 \\
(0.42,1.70)\end{array}$ & $\begin{array}{c}1.10 \\
(0.45,1.76)\end{array}$ & $\begin{array}{c}1.38 \\
(0.65,2.10)\end{array}$ & $\begin{array}{c}1.67 \\
(0.87,2.46)\end{array}$ & $\begin{array}{c}2.62 \\
(1.66,3.69)\end{array}$ & 1.00 & 1.04 & 1.30 & 1.57 & 2.52 \\
\hline $60-69$ & $\begin{array}{c}1.12 \\
(0.47,1.78)\end{array}$ & $\begin{array}{c}1.08 \\
(0.43,1.72)\end{array}$ & $\begin{array}{c}0.59 \\
(0.11,1.46)\end{array}$ & $\begin{array}{c}1.19 \\
(0.51,1.87)\end{array}$ & $\begin{array}{c}1.01 \\
(0.39,1.64)\end{array}$ & 1.00 & 0.96 & 0.53 & 1.06 & 0.90 \\
\hline $70-79$ & $\begin{array}{c}0.47 \\
(0.05,0.90)\end{array}$ & $\begin{array}{c}0.33 \\
(-0.03,0.68)\end{array}$ & $\begin{array}{c}0.88 \\
(0.30,1.46)\end{array}$ & $\begin{array}{c}0.49 \\
(0.06,0.92)\end{array}$ & $\begin{array}{c}1.16 \\
(0.49,1.83)\end{array}$ & 1.00 & 0.69 & 1.86 & 1.03 & 2.45 \\
\hline $80+$ & $\begin{array}{c}1.40 \\
(0.67,2.13)\end{array}$ & $\begin{array}{c}1.05 \\
(0.42,1.69)\end{array}$ & $\begin{array}{c}1.01 \\
(0.39,1.63)\end{array}$ & $\begin{array}{c}1.54 \\
(0.77,2.30)\end{array}$ & $\begin{array}{c}1.81 \\
(0.98,2.65)\end{array}$ & 1.00 & 0.75 & 0.72 & 1.10 & 1.30 \\
\hline \multicolumn{11}{|l|}{ Race } \\
\hline All & $\begin{array}{c}21.01 \\
(18.17,23.85)\end{array}$ & $\begin{array}{c}23.44 \\
(20.44,26.44)\end{array}$ & $\begin{array}{c}23.22 \\
(20.24,26.21)\end{array}$ & $\begin{array}{c}26.21 \\
(23.04,29.38)\end{array}$ & $\begin{array}{c}27.50 \\
(24.25,30.75)\end{array}$ & 1.00 & 1.12 & 1.11 & 1.25 & 1.31 \\
\hline 0-09 & $\begin{array}{c}81.60 \\
(76.00,87.20)\end{array}$ & $\begin{array}{c}87.16 \\
(81.37,92.95)\end{array}$ & $\begin{array}{c}90.42 \\
(84.53,96.32)\end{array}$ & $\begin{array}{c}98.44 \\
(92.29,104.59)\end{array}$ & $\begin{array}{c}98.58 \\
(92.42,104.73)\end{array}$ & 1.00 & 1.07 & 1.11 & 1.21 & 1.21 \\
\hline $10-19$ & $\begin{array}{c}55.08 \\
(50.48,59.68)\end{array}$ & $\begin{array}{c}62.42 \\
(57.52,67.31)\end{array}$ & $\begin{array}{c}57.88 \\
(53.17,62.60)\end{array}$ & $\begin{array}{c}66.51 \\
(61.46,71.57)\end{array}$ & $\begin{array}{c}71.21 \\
(65.98,76.44)\end{array}$ & 1.00 & 1.13 & 1.05 & 1.21 & 1.29 \\
\hline $20-29$ & $\begin{array}{c}7.40 \\
(5.71,9.08)\end{array}$ & $\begin{array}{c}10.06 \\
(8.09,12.03)\end{array}$ & $\begin{array}{c}10.68 \\
(8.65,12.71)\end{array}$ & $\begin{array}{c}12.62 \\
(10.42,14.83)\end{array}$ & $\begin{array}{c}14.19 \\
(11.86,16.53)\end{array}$ & 1.00 & 1.36 & 1.44 & 1.71 & 1.92 \\
\hline 30-39 & $\begin{array}{c}3.81 \\
(2.60,5.02)\end{array}$ & $\begin{array}{c}4.67 \\
(3.33,6.01)\end{array}$ & $\begin{array}{c}4.32 \\
(3.03,5.61)\end{array}$ & $\begin{array}{c}5.78 \\
(4.29,7.27)\end{array}$ & $\begin{array}{c}6.39 \\
(4.83,7.96)\end{array}$ & 1.00 & 1.23 & 1.13 & 1.52 & 1.68 \\
\hline $40-49$ & $\begin{array}{c}1.97 \\
(1.10,2.84)\end{array}$ & $\begin{array}{c}2.63 \\
(1.62,3.63)\end{array}$ & $\begin{array}{c}2.61 \\
(1.61,3.62)\end{array}$ & $\begin{array}{c}3.26 \\
(2.14,4.38)\end{array}$ & $\begin{array}{c}4.29 \\
(3.01,5.57)\end{array}$ & 1.00 & 1.33 & 1.33 & 1.65 & 2.18 \\
\hline $50-59$ & $\begin{array}{c}0.95 \\
(0.35,1.55)\end{array}$ & $\begin{array}{c}1.13 \\
(0.47,1.79)\end{array}$ & $\begin{array}{c}1.28 \\
(0.58,1.99)\end{array}$ & $\begin{array}{c}1.62 \\
(0.83,2.41)\end{array}$ & $\begin{array}{c}2.52 \\
(1.54,3.50)\end{array}$ & 1.00 & 1.19 & 1.35 & 1.70 & 2.65 \\
\hline
\end{tabular}




\begin{tabular}{|c|c|c|c|c|c|c|c|c|c|c|}
\hline $60-69$ & $\begin{array}{c}0.91 \\
(0.32,1.51)\end{array}$ & $\begin{array}{c}1.00 \\
(0.38,1.62)\end{array}$ & $\begin{array}{c}0.61 \\
(0.13,1.10)\end{array}$ & $\begin{array}{c}1.14 \\
(0.48,1.80)\end{array}$ & $\begin{array}{c}1.09 \\
(0.44,1.73)\end{array}$ & 1.00 & 1.10 & 0.67 & 1.24 & 1.19 \\
\hline 70-79 & $\begin{array}{c}0.37 \\
(-0.01,0.74)\end{array}$ & $\begin{array}{c}0.36 \\
(-0.01,0.73)\end{array}$ & $\begin{array}{c}0.90 \\
(0.31,1.49)\end{array}$ & $\begin{array}{c}0.34 \\
(-0.02,0.69)\end{array}$ & $\begin{array}{c}1.27 \\
(0.57,1.97)\end{array}$ & 1.00 & 0.98 & 2.46 & 0.92 & 3.48 \\
\hline $80+$ & $\begin{array}{c}1.33 \\
(0.62,2.05)\end{array}$ & $\begin{array}{c}1.15 \\
(0.48,1.8)\end{array}$ & $\begin{array}{c}0.94 \\
(0.34,1.55)\end{array}$ & $\begin{array}{c}1.52 \\
(0.75,2.28)\end{array}$ & $\begin{array}{c}1.52 \\
(0.75,2.28)\end{array}$ & 1.00 & 0.86 & 0.71 & 1.14 & 1.14 \\
\hline $\begin{array}{l}\text { African-American } \\
\text { All }\end{array}$ & $\begin{array}{c}27.54 \\
(24.29,30.79)\end{array}$ & $\begin{array}{c}31.83 \\
(28.3,35.32)\end{array}$ & $\begin{array}{c}34.75 \\
(31.10,38.40)\end{array}$ & $\begin{array}{c}41.10 \\
(37.13,45.08)\end{array}$ & $\begin{array}{c}49.69 \\
(45.32,54.06)\end{array}$ & 1.00 & 1.16 & 1.26 & 1.49 & 1.80 \\
\hline $0-09$ & $\begin{array}{c}81.04 \\
(75.47,86.62)\end{array}$ & $\begin{array}{c}88.65 \\
(82.81,94.48)\end{array}$ & $\begin{array}{c}101.20 \\
(94.97,107.44)\end{array}$ & $\begin{array}{c}108.37 \\
(102.89,115.86)\end{array}$ & $\begin{array}{c}141.56 \\
(134.18,148.93)\end{array}$ & 1.00 & 1.09 & 1.25 & 1.34 & 1.75 \\
\hline $10-19$ & $\begin{array}{c}49.07 \\
(44.72,53.41)\end{array}$ & $\begin{array}{c}60.91 \\
(56.07,65.75)\end{array}$ & $\begin{array}{c}64.98 \\
(59.98,69.98)\end{array}$ & $\begin{array}{c}86.40 \\
(80.64,92.17)\end{array}$ & $\begin{array}{c}93.02 \\
(87.04,98.99)\end{array}$ & 1.00 & 1.24 & 1.32 & 1.76 & 1.90 \\
\hline 20-29 & $\begin{array}{c}6.90 \\
(5.27,8.53)\end{array}$ & $\begin{array}{c}11.29 \\
(9.21,13.37)\end{array}$ & $\begin{array}{c}10.41 \\
(7.36,11.13)\end{array}$ & $\begin{array}{c}13.15 \\
(10.90,15.39)\end{array}$ & $\begin{array}{c}20.47 \\
(17.67,23.27)\end{array}$ & 1.00 & 1.64 & 1.51 & 1.91 & 2.97 \\
\hline 30-39 & $\begin{array}{c}7.44 \\
(5.75,9.13)\end{array}$ & $\begin{array}{c}10.48 \\
(8.47,12.48)\end{array}$ & $\begin{array}{c}9.24 \\
(7.36,11.13)\end{array}$ & $\begin{array}{c}10.30 \\
(8.31,12.29)\end{array}$ & $\begin{array}{c}8.99 \\
(7.13,10.84)\end{array}$ & 1.00 & 1.41 & 1.24 & 1.38 & 1.21 \\
\hline $40-49$ & $\begin{array}{c}5.91 \\
(4.40,7.42)\end{array}$ & $\begin{array}{c}4.48 \\
(3.17,5.79)\end{array}$ & $\begin{array}{c}4.64 \\
(3.30,5.97)\end{array}$ & $\begin{array}{c}6.41 \\
(4.84,7.98)\end{array}$ & $\begin{array}{c}8.21 \\
(6.44,9.99)\end{array}$ & 1.00 & 0.76 & 0.78 & 1.08 & 1.39 \\
\hline $50-59$ & $\begin{array}{c}2.33 \\
(1.38,3.27)\end{array}$ & $\begin{array}{c}1.00 \\
(0.38,1.61)\end{array}$ & $\begin{array}{c}2.96 \\
(1.90,4.03)\end{array}$ & $\begin{array}{c}2.62 \\
(1.61,3.62)\end{array}$ & $\begin{array}{c}5.24 \\
(3.82,6.65)\end{array}$ & 1.00 & 0.43 & 1.27 & 1.13 & 2.25 \\
\hline $60-69$ & $\begin{array}{c}2.87 \\
(1.82,3.92)\end{array}$ & $\begin{array}{c}2.29 \\
(1.35,3.23)\end{array}$ & $\begin{array}{c}0.57 \\
(0.10,1.04)\end{array}$ & $\begin{array}{c}2.26 \\
(1.33,3.19)\end{array}$ & $\begin{array}{c}0.57 \\
(0.10,1.03)\end{array}$ & 1.00 & 0.80 & 0.20 & 0.79 & 0.20 \\
\hline 70-79 & $\begin{array}{c}1.80 \\
(0.97,2.64)\end{array}$ & $\begin{array}{c}0.00 \\
(0.00,0.00)\end{array}$ & $\begin{array}{c}0.90 \\
(0.31,1.48)\end{array}$ & $\begin{array}{c}2.67 \\
(1.66,3.69)\end{array}$ & $\begin{array}{c}0.00 \\
(0.00,0.00)\end{array}$ & 1.00 & 0.00 & 0.50 & 1.48 & 0.00 \\
\hline $80+$ & $\begin{array}{c}2.21 \\
(1.29,3.14)\end{array}$ & $\begin{array}{c}0.00 \\
(0.00,0.00)\end{array}$ & $\begin{array}{c}2.20 \\
(1.28,3.12)\end{array}$ & $\begin{array}{c}2.19 \\
(1.28,3.11)\end{array}$ & $\begin{array}{c}6.58 \\
(4.99,8.17)\end{array}$ & 1.00 & 0.00 & 0.99 & 0.99 & 2.97 \\
\hline $\begin{array}{l}\text { Ethnicity } \\
\quad \text { Hispanic Origin }\end{array}$ & $\begin{array}{c}30.33 \\
(26.92,33.75)\end{array}$ & $\begin{array}{c}25.23 \\
(22.11,28.34)\end{array}$ & $\begin{array}{c}16.75 \\
(14.21,19.28)\end{array}$ & $\begin{array}{c}21.64 \\
(18.76,24.53)\end{array}$ & $\begin{array}{c}19.60 \\
(16 . .86,22.34)\end{array}$ & 1.00 & 0.83 & 0.55 & 0.71 & 0.65 \\
\hline Not of Hispanic Origin & $\begin{array}{c}20.23 \\
(17.45,23.02)\end{array}$ & $\begin{array}{c}19.37 \\
(16.64,22.10)\end{array}$ & $\begin{array}{c}17.59 \\
(14.99,20.19)\end{array}$ & $\begin{array}{c}17.10 \\
(14.54,19.66)\end{array}$ & $\begin{array}{c}15.99 \\
(13.51,18.47)\end{array}$ & 1.00 & 0.96 & 0.87 & 0.85 & 0.79 \\
\hline
\end{tabular}


Table 2.3: Time-trend Analysis of Rate of Sexual Assault among Males from 2001 to 2005

\begin{tabular}{cccccccc}
\hline \multicolumn{7}{c}{ Analysis Of Parameter Estimates } \\
\hline Parameter $^{\S}$ & $\boldsymbol{D F}$ & Estimate & Standard Error & Wald 95\% Confidence Limits & Chi-Square & Pr $>$ ChiSq \\
\hline Intercept & 1 & -6.2335 & 0.0245 & -6.2814 & -6.1856 & 64983.5 & $<.0001$ \\
Age & 1 & -0.9053 & 0.0122 & -0.9292 & -0.8814 & 5515.94 & $<.0001$ \\
Time & 1 & 0.0375 & 0.0070 & 0.0238 & 0.0512 & 28.91 & $<.0001$ \\
Age*Time & 1 & 0.0212 & 0.0034 & 0.0145 & 0.0279 & 38.29 & $<.0001$ \\
\hline \&age (1-9 represent the 9 age groups); time (1-5 representing years 2001-2005); age by time interaction &
\end{tabular}

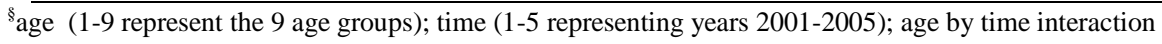

Table 2.4A: Time-trend Analysis of Rates of Sexual Assault among White Males from 2001 to 2005

\begin{tabular}{|c|c|c|c|c|c|c|c|}
\hline \multicolumn{8}{|c|}{ Analysis Of Parameter Estimates } \\
\hline Parameter $^{\S}$ & $D F$ & Estimate & Standard Error & Wald 95\% & ence Limits & Chi-Square & $\operatorname{Pr}>C h i S q$ \\
\hline Intercept & 1 & -6.0839 & 0.0267 & -6.1362 & -6.0316 & 51923.5 & $<.0001$ \\
\hline Age & 1 & -0.9425 & 0.0133 & -0.9687 & -0.9164 & 4991.00 & $<.0001$ \\
\hline Time & 1 & 0.0201 & 0.0076 & 0.0051 & 0.0350 & 6.88 & 0.0087 \\
\hline Age ${ }^{*}$ Time & 1 & 0.0262 & 0.0037 & 0.0189 & 0.0336 & 48.93 & $<.0001$ \\
\hline
\end{tabular}

Table 2.4B: Time-trend Analysis of Rates of Sexual Assault among African American Males from 2001 to 2005

\begin{tabular}{cccccccc}
\hline \multicolumn{7}{c}{ Analysis Of Parameter Estimates } \\
\hline Parameter $^{\S}$ & DF & Estimate & Standard Error & Wald 95\% Confidence Limits & Chi-Square & Pr $>$ ChiSq \\
\hline Intercept & 1 & -6.4979 & 0.0615 & -6.6185 & -6.3774 & 11163.6 & $<.0001$ \\
Age & 1 & -0.7568 & 0.0303 & -0.8163 & -0.6973 & 622.04 & $<.0001$ \\
Time & 1 & 0.1509 & 0.0172 & 0.1172 & 0.1846 & 77.14 & $<.0001$ \\
Age*Time & 1 & -0.0028 & 0.0085 & -0.0195 & 0.0138 & 0.11 & 0.7399 \\
\hline
\end{tabular}

\&age (1-9 represent the 9 age groups); time (1-5 representing years 2001-2005); age by time interaction 
Table 2.5: Characteristics of the Offender and Arrestee

\begin{tabular}{|c|c|c|c|c|c|c|c|c|c|c|}
\hline \multirow[t]{2}{*}{ Variables } & \multicolumn{5}{|c|}{$\begin{array}{c}\text { Offender } \\
N \\
\text { (Percentage) }\end{array}$} & \multicolumn{5}{|c|}{$\begin{array}{c}\text { Arrestee } \\
N \\
\text { (Percentage) }\end{array}$} \\
\hline & 2001 & 2002 & 2003 & 2004 & 2005 & 2001 & 2002 & 2003 & 2004 & 2005 \\
\hline \multicolumn{11}{|l|}{ Age Groups (in years) } \\
\hline $01-09$ & $\begin{array}{l}400 \\
(7.47)\end{array}$ & $\begin{array}{c}417 \\
(6.98)\end{array}$ & $\begin{array}{c}449 \\
(7.17)\end{array}$ & $\begin{array}{c}461 \\
(6.34)\end{array}$ & $\begin{array}{c}555 \\
(6.97)\end{array}$ & $\begin{array}{c}20 \\
(1.86)\end{array}$ & $\begin{array}{c}22 \\
(1.69)\end{array}$ & $\begin{array}{c}20 \\
(1.35)\end{array}$ & $\begin{array}{c}14 \\
(0.85)\end{array}$ & $\begin{array}{c}20 \\
(1.09)\end{array}$ \\
\hline $10-19$ & $\begin{array}{c}2349 \\
(43.85)\end{array}$ & $\begin{array}{c}2476 \\
(41.44)\end{array}$ & $\begin{array}{c}2661 \\
(42.50)\end{array}$ & $\begin{array}{c}3140 \\
(43.15)\end{array}$ & $\begin{array}{c}3327 \\
(41.76)\end{array}$ & $\begin{array}{c}561 \\
(52.23)\end{array}$ & $\begin{array}{c}586 \\
(45.15)\end{array}$ & $\begin{array}{c}694 \\
(46.92)\end{array}$ & $\begin{array}{c}827 \\
(50.15)\end{array}$ & $\begin{array}{c}902 \\
(49.18)\end{array}$ \\
\hline $20-29$ & $\begin{array}{c}808 \\
(15.08)\end{array}$ & $\begin{array}{c}909 \\
(15.21)\end{array}$ & $\begin{array}{c}993 \\
(15.86)\end{array}$ & $\begin{array}{c}1233 \\
(16.94)\end{array}$ & $\begin{array}{c}1338 \\
(16.79)\end{array}$ & $\begin{array}{c}147 \\
(13.69)\end{array}$ & $\begin{array}{c}175 \\
(13.48)\end{array}$ & $\begin{array}{c}229 \\
(15.48)\end{array}$ & $\begin{array}{c}252 \\
(15.28)\end{array}$ & $\begin{array}{c}320 \\
(17.45)\end{array}$ \\
\hline $30-39$ & $\begin{array}{c}885 \\
(16.52)\end{array}$ & $\begin{array}{c}1052 \\
(17.61)\end{array}$ & $\begin{array}{c}950 \\
(15.17)\end{array}$ & $\begin{array}{c}1117 \\
(15.35)\end{array}$ & $\begin{array}{c}1153 \\
(14.47)\end{array}$ & $\begin{array}{c}148 \\
(13.78)\end{array}$ & $\begin{array}{c}272 \\
(20.96)\end{array}$ & $\begin{array}{c}198 \\
(13.39)\end{array}$ & $\begin{array}{c}247 \\
(14.98)\end{array}$ & $\begin{array}{c}248 \\
(13.52)\end{array}$ \\
\hline $40-49$ & $\begin{array}{c}559 \\
(10.43)\end{array}$ & $\begin{array}{c}660 \\
(11.05)\end{array}$ & $\begin{array}{c}667 \\
(10.65)\end{array}$ & $\begin{array}{c}779 \\
(10.70)\end{array}$ & $\begin{array}{c}865 \\
(10.86)\end{array}$ & $\begin{array}{c}116 \\
(10.80)\end{array}$ & $\begin{array}{c}146 \\
(11.25)\end{array}$ & $\begin{array}{c}154 \\
(10.41)\end{array}$ & $\begin{array}{c}185 \\
(11.22)\end{array}$ & $\begin{array}{c}193 \\
(10.52)\end{array}$ \\
\hline $50-59$ & $\begin{array}{c}225 \\
(4.20)\end{array}$ & $\begin{array}{c}290 \\
(4.85)\end{array}$ & $\begin{array}{c}302 \\
(4.82)\end{array}$ & $\begin{array}{c}345 \\
(4.74)\end{array}$ & $\begin{array}{c}451 \\
(5.66)\end{array}$ & $\begin{array}{c}47 \\
(4.38)\end{array}$ & $\begin{array}{c}66 \\
(5.08)\end{array}$ & $\begin{array}{c}76 \\
(5.14)\end{array}$ & $\begin{array}{c}81 \\
(4.91)\end{array}$ & $\begin{array}{c}88 \\
(4.80)\end{array}$ \\
\hline $60-69$ & $\begin{array}{c}81 \\
(1.51)\end{array}$ & $\begin{array}{c}126 \\
(2.11)\end{array}$ & $\begin{array}{c}131 \\
(2.09)\end{array}$ & $\begin{array}{c}146 \\
(2.01)\end{array}$ & $\begin{array}{c}191 \\
(2.40)\end{array}$ & $\begin{array}{c}24 \\
(2.23)\end{array}$ & $\begin{array}{c}23 \\
(1.77)\end{array}$ & $\begin{array}{c}32 \\
(2.16)\end{array}$ & $\begin{array}{c}30 \\
(1.82)\end{array}$ & $\begin{array}{c}43 \\
(2.34)\end{array}$ \\
\hline $70-79$ & $\begin{array}{c}33 \\
(0.62)\end{array}$ & $\begin{array}{c}29 \\
(0.49)\end{array}$ & $\begin{array}{c}99 \\
(1.58)\end{array}$ & $\begin{array}{c}41 \\
(0.56)\end{array}$ & $\begin{array}{c}64 \\
(0.80)\end{array}$ & $\begin{array}{c}8 \\
(0.74)\end{array}$ & $\begin{array}{c}8 \\
(0.62)\end{array}$ & $\begin{array}{c}76 \\
(5.14)\end{array}$ & $\begin{array}{c}10 \\
(0.61)\end{array}$ & $\begin{array}{c}19 \\
(1.04)\end{array}$ \\
\hline $80+$ & $\begin{array}{c}17 \\
(0.32) \\
\end{array}$ & $\begin{array}{c}16 \\
(0.27) \\
\end{array}$ & $\begin{array}{c}9 \\
(0.14) \\
\end{array}$ & $\begin{array}{c}15 \\
(0.21) \\
\end{array}$ & $\begin{array}{c}23 \\
(0.29) \\
\end{array}$ & $\begin{array}{c}3 \\
(0.28) \\
\end{array}$ & $\begin{array}{c}0 \\
(0.0) \\
\end{array}$ & $\begin{array}{c}0 \\
(0.0) \\
\end{array}$ & $\begin{array}{c}3 \\
(0.18) \\
\end{array}$ & $\begin{array}{c}1 \\
(0.04) \\
\end{array}$ \\
\hline \multicolumn{11}{|l|}{ Gender } \\
\hline $\begin{array}{l}\text { Male } \\
\text { Female }\end{array}$ & $\begin{array}{c}4690 \\
(83.05) \\
957 \\
(16.95) \\
\end{array}$ & $\begin{array}{c}5220 \\
(83.29) \\
1047 \\
(16.71) \\
\end{array}$ & $\begin{array}{c}5514 \\
(83.40) \\
1097 \\
(16.59) \\
\end{array}$ & $\begin{array}{c}6428 \\
(83.68) \\
1254 \\
(16.32) \\
\end{array}$ & $\begin{array}{c}7027 \\
(83.00) \\
1439 \\
(17.00) \\
\end{array}$ & $\begin{array}{c}943 \\
(87.64) \\
133 \\
(12.36) \\
\end{array}$ & $\begin{array}{c}1141 \\
(87.77) \\
159 \\
(12.23) \\
\end{array}$ & $\begin{array}{c}1300 \\
(87.84) \\
180 \\
(12.16) \\
\end{array}$ & $\begin{array}{c}1418 \\
(85.89) \\
233 \\
(14.11) \\
\end{array}$ & $\begin{array}{c}1580 \\
(86.10) \\
255 \\
(13.90) \\
\end{array}$ \\
\hline \multicolumn{11}{|l|}{ Race } \\
\hline White & $\begin{array}{c}4321 \\
(80.72)\end{array}$ & $\begin{array}{c}4870 \\
(81.93)\end{array}$ & $\begin{array}{c}4997 \\
(80.18)\end{array}$ & $\begin{array}{c}5778 \\
(79.83)\end{array}$ & $\begin{array}{c}6143 \\
(77.04)\end{array}$ & $\begin{array}{c}852 \\
(80.38)\end{array}$ & $\begin{array}{c}1096 \\
(85.23)\end{array}$ & $\begin{array}{c}1172 \\
(80.82)\end{array}$ & $\begin{array}{c}1301 \\
(80.31)\end{array}$ & $\begin{array}{c}1443 \\
(79.99)\end{array}$ \\
\hline African-American & $\begin{array}{c}979 \\
(18.29)\end{array}$ & $\begin{array}{c}1017 \\
(17.11)\end{array}$ & $\begin{array}{c}1185 \\
(19.01)\end{array}$ & $\begin{array}{c}1413 \\
(19.52)\end{array}$ & $\begin{array}{c}1732 \\
(21.72)\end{array}$ & $\begin{array}{c}198 \\
(18.68)\end{array}$ & $\begin{array}{c}173 \\
(13.45)\end{array}$ & $\begin{array}{c}263 \\
(18.38)\end{array}$ & $\begin{array}{c}302 \\
(18.64)\end{array}$ & $\begin{array}{c}341 \\
(18.90)\end{array}$ \\
\hline $\begin{array}{l}\text { American Indian/Alaskan } \\
\text { Native }\end{array}$ & $\begin{array}{c}21 \\
(0.39)\end{array}$ & $\begin{array}{c}24 \\
(0.40)\end{array}$ & $\begin{array}{c}24 \\
(0.38)\end{array}$ & $\begin{array}{c}29 \\
(0.40)\end{array}$ & $\begin{array}{c}52 \\
(0.65)\end{array}$ & $\begin{array}{c}7 \\
(0.66)\end{array}$ & $\begin{array}{c}6 \\
(0.47)\end{array}$ & $\begin{array}{c}8 \\
(0.55)\end{array}$ & $\begin{array}{c}10 \\
(0.62)\end{array}$ & $\begin{array}{c}7 \\
(0.39)\end{array}$ \\
\hline Asian/Pacific Islander & $\begin{array}{c}32 \\
(0.60)\end{array}$ & $\begin{array}{c}33 \\
(0.56)\end{array}$ & $\begin{array}{c}26 \\
(0.42)\end{array}$ & $\begin{array}{c}18 \\
(0.25)\end{array}$ & $\begin{array}{c}47 \\
(0.59)\end{array}$ & $\begin{array}{c}3 \\
(0.28)\end{array}$ & $\begin{array}{c}11 \\
(0.86)\end{array}$ & $\begin{array}{c}7 \\
(0.48)\end{array}$ & $\begin{array}{c}7 \\
(0.43)\end{array}$ & $\begin{array}{c}13 \\
0.72)\end{array}$ \\
\hline
\end{tabular}


Table 2.6: Sexual Assault Incident Characteristics

\begin{tabular}{|c|c|c|c|c|c|}
\hline \multirow[t]{2}{*}{ Variables } & \multicolumn{5}{|c|}{$\begin{array}{c}N \\
\text { (Percentage) }\end{array}$} \\
\hline & 2001 & 2002 & 2003 & 2004 & 2005 \\
\hline \multicolumn{6}{|l|}{ Type } \\
\hline Forcible rape & $\begin{array}{c}314 \\
(5.81)\end{array}$ & $\begin{array}{c}350 \\
(5.78)\end{array}$ & $\begin{array}{c}366 \\
(5.68)\end{array}$ & $\begin{array}{c}394 \\
(5.27)\end{array}$ & $\begin{array}{c}453 \\
(5.53)\end{array}$ \\
\hline Forcible sodomy & $\begin{array}{c}1796 \\
(33.20)\end{array}$ & $\begin{array}{c}1932 \\
(31.89)\end{array}$ & $\begin{array}{c}2001 \\
(31.07)\end{array}$ & $\begin{array}{c}2307 \\
(30.88)\end{array}$ & $\begin{array}{c}2520 \\
(30.77)\end{array}$ \\
\hline Sexual assault with an object & $\begin{array}{c}254 \\
(4.70)\end{array}$ & $\begin{array}{c}294 \\
(4.85)\end{array}$ & $\begin{array}{c}268 \\
(4.16)\end{array}$ & $\begin{array}{c}329 \\
(4.40)\end{array}$ & $\begin{array}{c}357 \\
(4.36)\end{array}$ \\
\hline Forcible fondling & $\begin{array}{c}2585 \\
(52.84)\end{array}$ & $\begin{array}{c}3259 \\
(53.79)\end{array}$ & $\begin{array}{c}3506 \\
(54.44)\end{array}$ & $\begin{array}{c}4018 \\
(53.78)\end{array}$ & $\begin{array}{c}4376 \\
(53.42)\end{array}$ \\
\hline Incest & $\begin{array}{c}68 \\
(1.26)\end{array}$ & $\begin{array}{c}100 \\
(1.65)\end{array}$ & $\begin{array}{c}144 \\
(2.24)\end{array}$ & $\begin{array}{c}184 \\
(2.46)\end{array}$ & $\begin{array}{c}247 \\
(3.02)\end{array}$ \\
\hline Statutory rape & $\begin{array}{c}119 \\
(2.20)\end{array}$ & $\begin{array}{c}124 \\
(2.05)\end{array}$ & $\begin{array}{c}155 \\
(2.41) \\
\end{array}$ & $\begin{array}{c}239 \\
(3.20) \\
\end{array}$ & $\begin{array}{c}238 \\
(2.91) \\
\end{array}$ \\
\hline \multicolumn{6}{|l|}{ Location } \\
\hline Public Place & $\begin{array}{c}92 \\
(1.70)\end{array}$ & $\begin{array}{c}115 \\
(1.90)\end{array}$ & $\begin{array}{c}108 \\
(1.68)\end{array}$ & $\begin{array}{c}138 \\
(1.85)\end{array}$ & $\begin{array}{c}197 \\
(2.41)\end{array}$ \\
\hline Commercial Place & $\begin{array}{c}264 \\
(4.88)\end{array}$ & $\begin{array}{c}279 \\
(4.60)\end{array}$ & $\begin{array}{c}264 \\
(4.10)\end{array}$ & $\begin{array}{c}365 \\
(4.89)\end{array}$ & $\begin{array}{c}419 \\
(5.12)\end{array}$ \\
\hline Outdoors & $\begin{array}{c}263 \\
(4.86)\end{array}$ & $\begin{array}{c}257 \\
(4.24)\end{array}$ & $\begin{array}{c}276 \\
(4.29)\end{array}$ & $\begin{array}{c}340 \\
(4.55)\end{array}$ & $\begin{array}{c}393 \\
(4.80)\end{array}$ \\
\hline Residence & $\begin{array}{c}4040 \\
(74.69)\end{array}$ & $\begin{array}{c}4535 \\
(74.85)\end{array}$ & $\begin{array}{c}4918 \\
(76.37)\end{array}$ & $\begin{array}{c}5498 \\
(73.59)\end{array}$ & $\begin{array}{c}6010 \\
(73.37)\end{array}$ \\
\hline School/College & $\begin{array}{c}174 \\
(3.22)\end{array}$ & $\begin{array}{c}216 \\
(3.51)\end{array}$ & $\begin{array}{c}204 \\
(3.17)\end{array}$ & $\begin{array}{c}311 \\
(4.16)\end{array}$ & $\begin{array}{c}289 \\
(3.53)\end{array}$ \\
\hline Jail/Prison & $\begin{array}{c}123 \\
(2.27)\end{array}$ & $\begin{array}{c}117 \\
(1.93)\end{array}$ & $\begin{array}{c}119 \\
(1.85)\end{array}$ & $\begin{array}{c}202 \\
(2.70)\end{array}$ & $\begin{array}{l}225 \\
(2.75)\end{array}$ \\
\hline Unknown & $\begin{array}{c}453 \\
(8.37) \\
\end{array}$ & $\begin{array}{c}540 \\
(8.91) \\
\end{array}$ & $\begin{array}{c}551 \\
(8.56) \\
\end{array}$ & $\begin{array}{c}617 \\
(8.26) \\
\end{array}$ & $\begin{array}{c}658 \\
(8.03) \\
\end{array}$ \\
\hline \multicolumn{6}{|l|}{ Use of Weapon } \\
\hline Firearm/Sharp object & $\begin{array}{c}30 \\
(0.62)\end{array}$ & $\begin{array}{c}51 \\
(0.94)\end{array}$ & $\begin{array}{c}38 \\
(0.66)\end{array}$ & $\begin{array}{c}62 \\
(0.93)\end{array}$ & $\begin{array}{c}45 \\
(0.62)\end{array}$ \\
\hline Personal force (hands, feet, etc.) & $\begin{array}{c}2999 \\
(62.30)\end{array}$ & $\begin{array}{c}3376 \\
(62.53)\end{array}$ & $\begin{array}{c}3571 \\
(61.94)\end{array}$ & $\begin{array}{c}4019 \\
(60.07)\end{array}$ & $\begin{array}{c}4452 \\
(61.37)\end{array}$ \\
\hline Drugs/Narcotics & $\begin{array}{c}0 \\
(0.0)\end{array}$ & $\begin{array}{c}3 \\
(0.06)\end{array}$ & $\begin{array}{c}3 \\
(0.05)\end{array}$ & $\begin{array}{c}8 \\
(0.12)\end{array}$ & $\begin{array}{c}17 \\
(0.23)\end{array}$ \\
\hline Other & $\begin{array}{c}230 \\
(4.78)\end{array}$ & $\begin{array}{c}295 \\
(5.46)\end{array}$ & $\begin{array}{c}286 \\
(4.96)\end{array}$ & $\begin{array}{c}278 \\
(4.15)\end{array}$ & $\begin{array}{c}295 \\
(4.07)\end{array}$ \\
\hline None & $\begin{array}{c}1555 \\
(32.30)\end{array}$ & $\begin{array}{c}1674 \\
(31.01)\end{array}$ & $\begin{array}{c}1867 \\
(32.39)\end{array}$ & $\begin{array}{c}2324 \\
(34.73)\end{array}$ & $\begin{array}{c}2445 \\
(33.71)\end{array}$ \\
\hline
\end{tabular}


Figure 2.1: Five Year Annual Average of Male Sexual Assault Offenses by Offender (A) and Arrestee (B) Gender
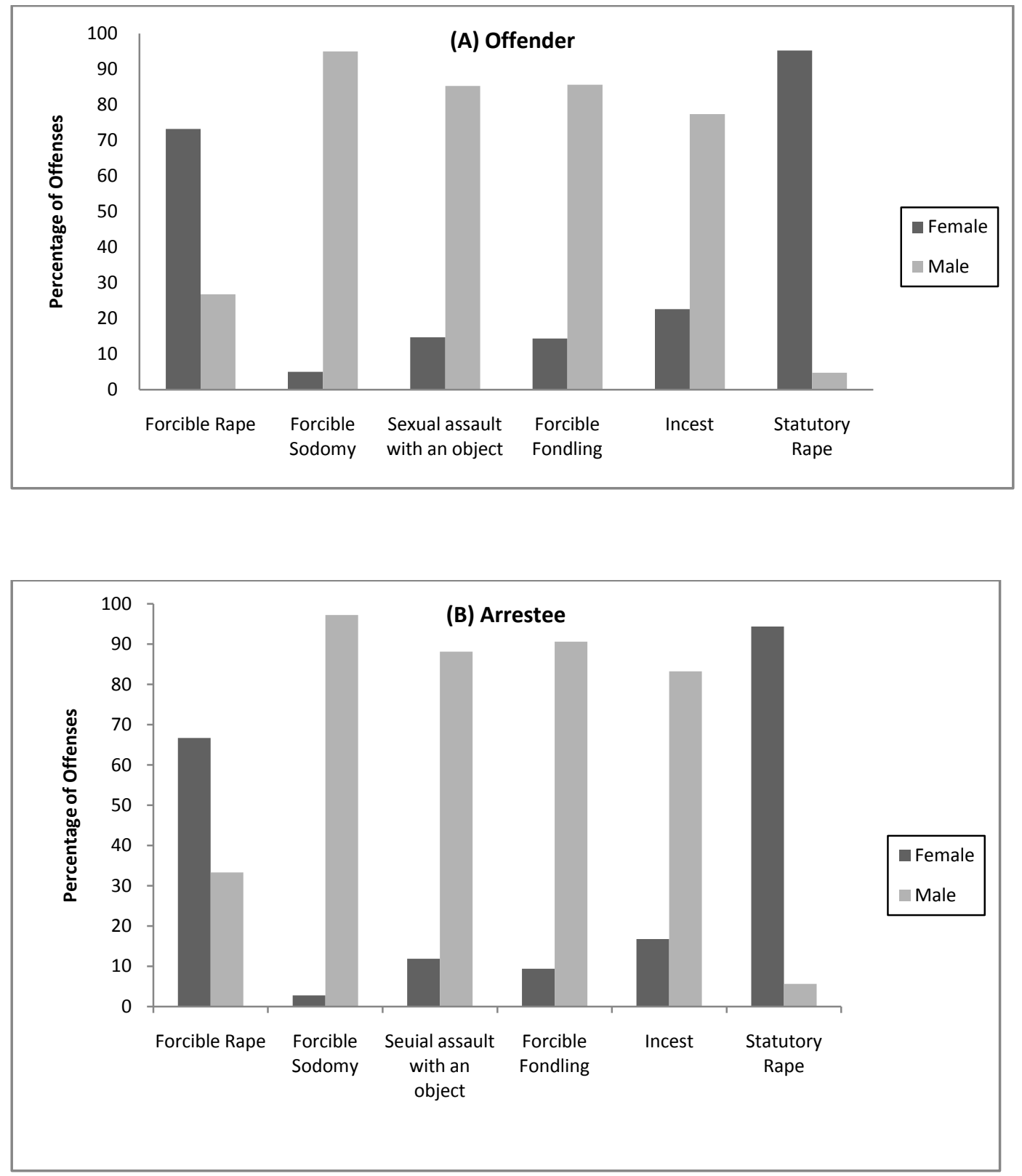
Table 2.7: Average Annual Sexual Violence Injury Rate by Type and Age Group

\begin{tabular}{c|cccc}
\hline & \multicolumn{4}{|c}{$\begin{array}{c}\text { Sexual Assault Rate per 100,000 Males } \\
\text { (95\% CI) }\end{array}$} \\
\hline Age & No Injuries & Minor & Major & Any \\
\hline $\mathbf{0 - 0 9}$ & 77.38 & 8.05 & 2.07 & 10.12 \\
& $(71.93,82.83)$ & $(6.29,9.81)$ & $(1.18,2.96)$ & $(8.15,12.09)$ \\
$\mathbf{1 0 - 1 9}$ & 52.04 & 4.45 & 0.99 & 5.44 \\
& $(47.57,56.51)$ & $(3.15,5.76)$ & $(0.37,1.61)$ & $(4.00,6.89)$ \\
$\mathbf{2 0 - 2 9}$ & 8.03 & 1.65 & 0.40 & 2.05 \\
& $(6.28,9.79)$ & $(0.85,2.45)$ & $(0.01,0.80)$ & $(1.16,2.94)$ \\
$\mathbf{3 0 - 3 9}$ & 4.10 & 0.75 & 0.23 & 0.98 \\
& $(2.85,5.36)$ & $(0.22,1.29)$ & $(-0.07,0.52)$ & $(0.37,1.60)$ \\
$\mathbf{4 0 - 4 9}$ & 2.40 & 0.49 & 0.15 & 0.64 \\
\multirow{2}{*}{$\mathbf{5 0 - 5 9}$} & $(1.44,3.37)$ & $(0.06,0.92)$ & $(-0.09,0.40)$ & $0.15,1.14)$ \\
& 1.10 & 0.23 & 0.16 & 0.39 \\
$\mathbf{6 0 - 6 9}$ & $(0.45,1.75)$ & $(-0.07,0.52)$ & $(-0.09,0.41)$ & $(0.00,0.77)$ \\
& 0.75 & 0.11 & 0.05 & 0.16 \\
$\mathbf{7 0 - 7 9}$ & $(0.21,1.29)$ & $(-0.10,0.32)$ & $(-0.09,0.20)$ & $(-0.09,0.42)$ \\
& 0.57 & 0.08 & 0.04 & 0.11 \\
$\mathbf{8 0 +}$ & $(0.10,1.04)$ & $(-0.09,0.25)$ & $(-0.08,0.16)$ & $(-0.10,0.32)$ \\
& 1.40 & 0.09 & 0.00 & 0.09 \\
& $(0.66,2.13)$ & $(-0.10,0.27)$ & $(0.00)$ & $(-0.10,0.27)$ \\
\hline
\end{tabular}

Figure 2.2: Average Annual Sexual Violence Injury Rate by Type and Age Group

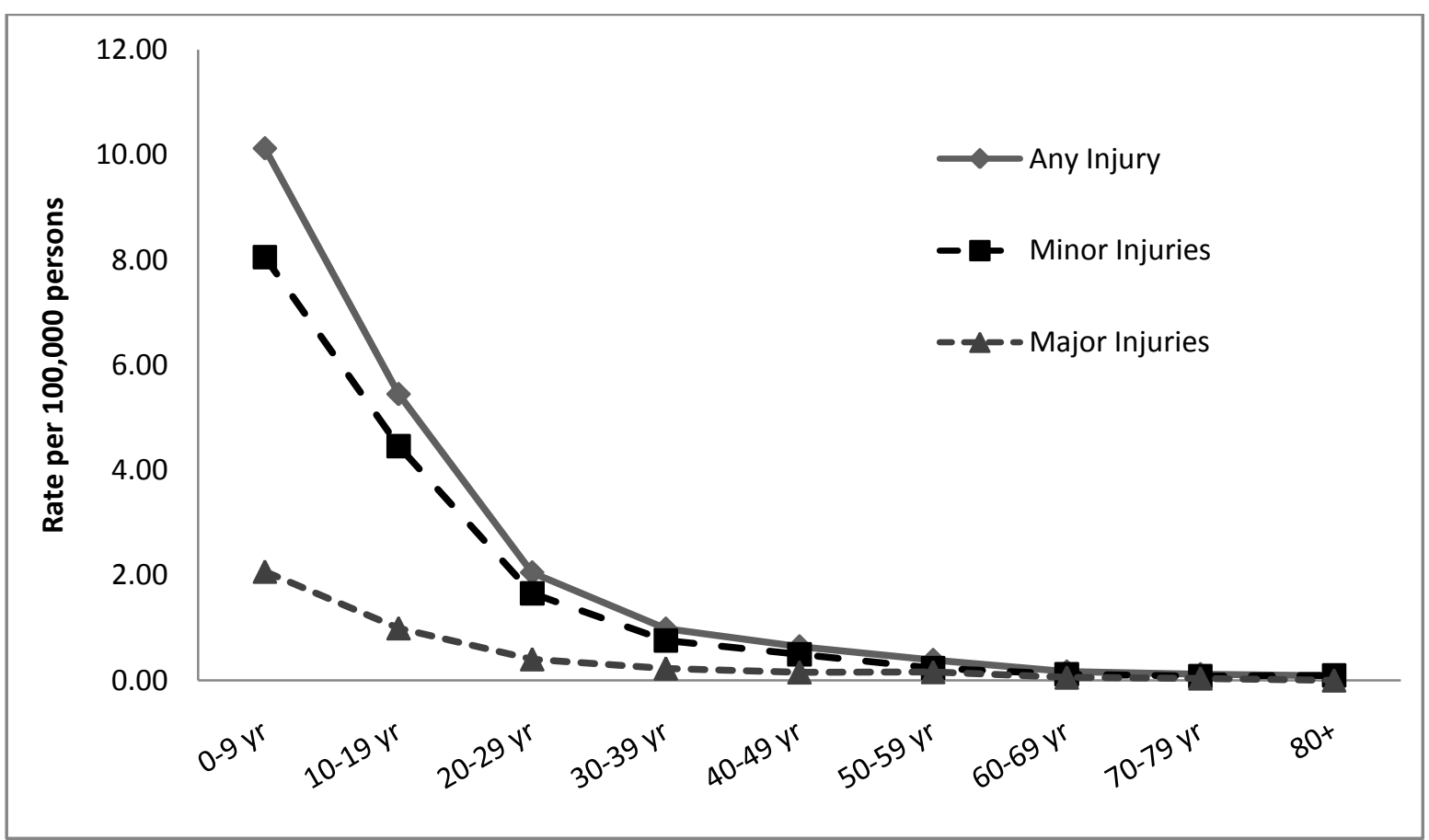


Figure 2.3: Time* of Incident of Male Sexual Assault from 2001 to 2005

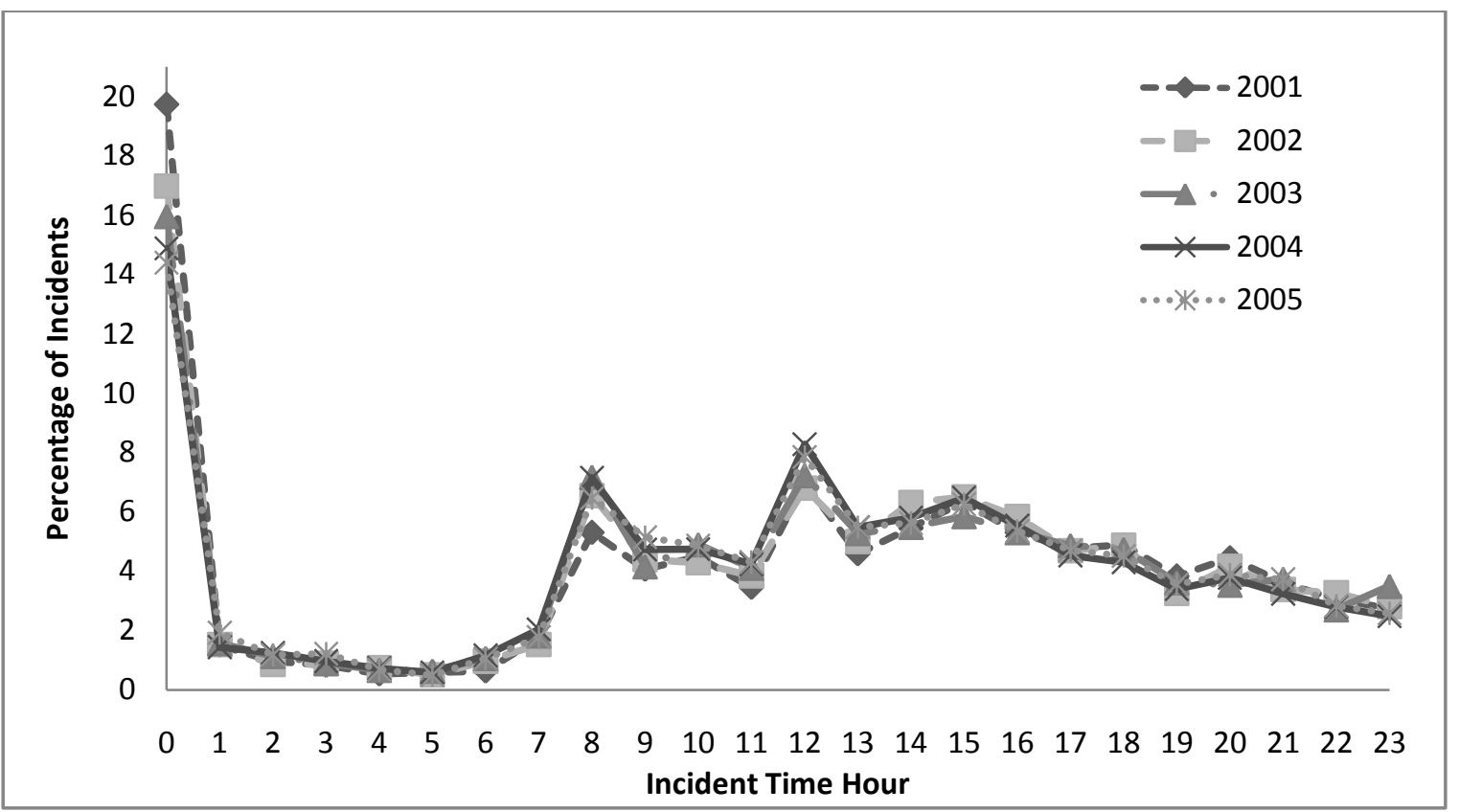

* NIBRS used military 24-hour time when reporting time of incident, therefore, if a range of time is given, for example if incident occurred between midnight to $3 \mathrm{am}$, the incident record will indicate the time as " 00 ". 
Chapter 3 


\section{Chapter 3}

\subsection{Introduction}

Sexual victimization refers to sexual activity where consent is not obtained or freely given. Sexual victimization among men and women is a serious public health problem that has been associated with long-lasting negative mental and physical health outcomes. ${ }^{71,126,127}$ Previous estimates have suggested that 1 in 6 women and 1 in 33 men will experience an attempted or completed rape at some point in their lifetime in the United States. ${ }^{4}$ Studies using adult samples have indicated that sexual victimization is a relatively frequent event, with prevalence estimates of sexual victimization ranging from $13 \%$ to $25 \%{ }^{4,27,128}$ among females and $0.6 \%$ to $22 \%$ 5, 13, 69 among males. Various studies have indicated that the prevalence of sexual violence victimization is higher among college-attending males and females (average age 20 years) when compared with other age groups. ${ }^{26,27,122}$ These studies have provided information on the prevalence and correlates of various types of sexual assault; however, gender-rooted differences in definitions of sexual violence may limit direct comparisons.

Sexual victimization has been associated with both long- and short-term poor mental health outcomes in both males and females. However, comparably fewer studies have evaluated possible gender differences in poor mental health outcomes associated with sexual victimization. The consequences of sexual assault among men and women appear to be similar (e.g., experiences of fear, humiliation, and anger at the time of incident). ${ }^{20,126}$ In a recent review of existing literature, Tewksbury (2007) reported that men who were sexually victimized were more likely to report psychological problems (e.g., depression, anxiety, substance abuse) when compared with non-victimized males. ${ }^{12}$ Also, men who were victimized as children were more 
likely to report mental health problems such as anxiety disorders, depression, sleep disturbances, and deliberate self-harm, when compared with adult victims. ${ }^{41}$

It is unclear whether the emotional impact of sexual victimization in males and females is similar, ${ }^{7,20}$ however, existing evidence suggests there may be considerable overlap. Rape trauma syndrome, in which depression, anger, guilt, and suicidal feelings can be experienced for years after an assault, has been documented among both male and female victims of sexual assault. ${ }^{99}$, ${ }^{129}$ More specifically, severe depression is a common problem reported by sexually assaulted males and females. ${ }^{15,45,46}$ Some studies have reported that male victims are more likely than female victims to present with severe depression and anxiety. ${ }^{13,39}$ In addition to depression and social isolation, increased anger and hostility have also been observed among male victims. ${ }^{39,42}$ Another behavior associated with depression among young adults is deliberate self-harm (DSH). ${ }^{61}$ In a recent review of literature by Mangnall et al. (2008), sexual trauma (mainly during childhood) was identified as a predisposing factor for $\mathrm{DSH}^{6}{ }^{63}$ Some studies have also suggested that men and women with a history of childhood sexual abuse may have harmed themselves as children. However, it is possible that victimized adolescents may use DSH as a mechanism for the release of tension and stress, and similar associations may not be observed in adult samples. ${ }^{59,109}$ Campbell et al. (2007) found a considerably higher prevalence of DSH and mental health problems among victimized females. However, existing studies of the psychological consequences of sexual assault among adult males have not examined the prevalence or association of DSH with sexual assault. Some factors that have been linked with repetitive DSH among young adults include psychological distress and "depressive tendencies," which may include major depression and suicide ideation. ${ }^{61-63}$ 
Depression is often associated with suicide ideation/attempts and hostility among victimized males. ${ }^{18,48}$ Suicide ideation alone is considered an important correlate of DSH among both males and females. ${ }^{59,60}$ A recent review of literature on male sexual assault suggested that suicide attempts are more common among adolescent and young adult victims. ${ }^{12}$ Juon and Ensminger (1997) reported that adverse life events such as sexual abuse are risk factors commonly associated with youth suicide. ${ }^{49}$ In a study of college students, the rate of suicide among undergraduate women was 3.4/100,000/year, whereas among undergraduate men, this rate was 9.1/100,000/year. ${ }^{54} \mathrm{~A}$ study of college-attending male victims of sexual assault suggested that $46 \%$ of all victims reported suicide ideation, $35 \%$ attempted suicide post-assault, $55 \%$ reported increased anger or rage, and $63.6 \%$ reported increased interpersonal problems. ${ }^{5} \mathrm{~A}$ study by Waldrop et al. (2007) investigating the interrelationships among victimization, PTSD, major depressive disorder, substance abuse, and suicidal behavior, reported that young adults with a history of sexual victimization were more likely to report suicide ideation and suicide attempts. ${ }^{50}$ Findings from a cross-sectional survey of adult males and females (16 years or older) in the general population indicated that exposure to sexual abuse was significantly related to suicidal behaviors. ${ }^{51}$ Though the relationships between sexual victimization and depression, ${ }^{52,53}$ depression and suicide ideation/attempt, ${ }^{54,55}$ and sexual assault and suicidal behaviors, ${ }^{56-58}$ are well-established among females, the relationships between sexual assault and suicide ideation among victimized males are not as well documented.

Furthermore, the coping strategies of victimized males may be different from females. General Strain Theory (GST) posits that certain strains or emotional stressors will increase the likelihood of aggressive behavior. ${ }^{64}$ Victimized males may more commonly blame themselves and report more hostility and anger when compared with female victims. ${ }^{6}$ Studies have suggested that the 
long-term impacts of sexual assault on men are likely to result in increased expressions of anger, hostility, and aggression, which have also been associated with an increased risk of suicidal or violent behaviors. $^{7,20}$ Existing studies have also confirmed an association between sexual assault and violent behavior among victims. ${ }^{65-67}$ Thus, GST may also explain gender differences in behavioral outcomes by identifying ways males may respond to experienced strain with anger (an externalizing emotion) whereas females may have a greater tendency to respond to strain with depressed mood (an internalizing emotion). ${ }^{64,68}$ Sigfusdottir et al. (2008) examined the mediating effect of depression and anger in the relationships between sexual abuse and suicidal behavior and delinquency. ${ }^{68}$ Their findings suggested that anger resulting from sexual abuse was more strongly and positively related to outwardly directed forms of delinquency, such as more use of physical violence among young males than females. These findings underscore the need for future research to investigate the relationship between the history of sexual assault and violent behavior among males.

Previous research suggests that sexual violence victimization is associated with risk behaviors such as alcohol, substance abuse, and adverse health outcomes in the long-term. ${ }^{29,47,126}$ The adverse consequences of sexual victimization range from depression to suicidal behavior, and their interrelationships are well documented. ${ }^{54,55,64}$ However, the gender differences in sexual victimization and self-harm behaviors (suicidal behavior and DSH) are not well understood. Additionally, various studies suggest that men are at greater risk of aggressive behaviors when compared to women. ${ }^{64,65}$ However, the associations between sexual victimization and externalizing behaviors among males have not been examined with similar granularity.

Another important aspect of sexual assault is how it is defined. Various studies that have estimated the prevalence of sexual assault or examined associated adverse health outcomes have 
used different definitions of sexual assault. Among females, the most commonly used definition of sexual assault includes rape or sexual harassment. However, studies have not examined in detail what men consider or define as sexual assault. In order to understand the adverse consequences associated with sexual victimization, the current project investigated the prevalence and correlates of sexual assault victimization among males.

Due to the large number of measures and analytic models used to identify these relationships, results from this study have been divided into three parts. First, the prevalence of risk behaviors, negative health outcomes, and sexual victimization among college-attending males and females is presented. Next, the differences between aggressive behaviors and sexual victimization among males and females are compared. The following hypotheses were tested in this second domain: (1) victimized males are more likely to perpetrate non-partner interpersonal violence when compared with non-victimized males; (2) victimized males are more likely to perpetrate intimate partner violence when compared with non-victimized males. Finally, associations between suicidal behavior, DSH, and sexual violence among victimized males and female are examined. This study was conducted to examine the following hypotheses: (3) victimized males are at a greater risk of suicidal behavior when compared with victimized females; (4) victimized males are at lower risk of deliberate self-harm when compared with victimized females.

\subsection{Methods}

\subsubsection{Participants}

A self-administered survey was given to students taking undergraduate psychology courses at a large public university in the Mid-Atlantic region of the United States. The survey was administered from December 2008 to March 2009, with potential participants in four undergraduate psychology classes (i.e., Introduction to Psychology, Introduction to Human 
Development, Introduction to Social Psychology, and Introduction to Abnormal Psychology).

Students learned about the research opportunity through a brief explanation of the study during class and by way of an informational flyer displayed on the Department's bulletin board. A total of 780 students participated in the study. Sixty participants were excluded from data analyses due to missing data on sexual violence victimization and basic demographic information resulting in an analytic sample of 720 participants (194 males and 526 females). As indicated by the administrative office of the Department of Psychology (verbal communication), the approximate 1:2.5 male-to-female ratio reflects the gender enrollment in undergraduate psychology courses. Participants received extra-credit for completing the self-administered questionnaire via an online research system. ${ }^{130}$ The survey took approximately 30 minutes to complete. Students consented to participation via the online system, and were given access to information about their rights as participants in the study. This study was approved by West Virginia University's Institutional Review Board (IRB).

\subsubsection{Measures}

The survey used standardized items and previously developed and validated scales to measure sexual victimization and associated risk behaviors (Appendix A). Relationships between unwanted sexual experiences, and physical and mental health outcomes, and risk behaviors were examined. In addition to sexual violence victimization, each participant was asked about general health status, risk behaviors, depression or anxiety, deliberate self-harm, suicide ideation/attempt, interpersonal violence, and intimate partner violence. All participants were asked to identify demographic characteristics including age, gender, race, year in school, relationship status, and sexual orientation. 
Sexual victimization questions were adapted from the Sexual Experience Survey (SES); the most frequently used measure of unwanted sexual experience. ${ }^{131,132}$ The SES includes various items measuring a range of unwanted sexual experiences. Sexual violence victimization in this study was divided into eight main categories (see Appendix B) and included sex play, oral, vaginal, and anal sex as well as manual stimulation. Participants were specifically asked if they had experienced any unwanted sexual activity with a man or woman who used continual argument, intimidation, pressure, physical force, and/or alcohol or drugs. The participants were also asked if they consumed alcohol or drugs willingly, a factor that may have impaired their ability to resist unwanted sexual activity. Based on their responses to questions about sexual assault, participants were categorized into victimized and non-victimized groups.

Health outcomes included as correlates of sexual victimization included poor or fair self-reported health status, six or more days of poor mental health, overall life satisfaction, and activity limitations due to any physical, mental, or emotional health problems. Specific questions about health outcomes included: (1) "Would you say that in general your health is __.." Response options included excellent, good, fair or poor. Self-reported health status was re-coded to create a dichotomous measure of health including good (combining "Excellent and Good") and poor or fair categories. (2) The number of days with poor mental health was calculated based on responses to the question "Now thinking about your mental health, which includes stress, depression, and problems with emotions, for how many days during the past 30 days was your mental health not good?" Response options included 0 days, 1-5 days, 6-9 days, and 10+ days. The poor mental health status was re-coded to create a dichotomous measure of $<6$ days and $\geq 6$ days with poor mental health. (3) Life satisfaction was measured using responses to the question "In general, how satisfied are you with your life?" Response options for this question included 
very satisfied, satisfied, dissatisfied and very dissatisfied. Life satisfaction was re-coded into a dichotomous measure including satisfied (very satisfied and satisfied) and not satisfied (dissatisfied and very dissatisfied) categories. (4) Activity limitations due to any health problems were measured by the question asking "Are you limited in any way in any activities because of physical, mental, or emotional problems?" Response options to the question asking about activity limitations included yes and no.

Risk behavior (alcohol \& substance abuse) questions were adapted from the American College Health Association's National College Health Assessment survey. ${ }^{133}$ Participants were asked about tobacco, alcohol, marijuana, cocaine, and other drug use in the past 30 days. In order to measure heavy episodic drinking, respondents were also asked if they consumed 5 or more alcoholic drinks on one occasion in the past 30 days. Additional details about the generalizability and reliability of these measures are available from the American College Health Association. ${ }^{133}$

Depressive symptomology was measured by the Patient Health Questionnaire 8 (PHQ-8). While a modification of the original PHQ-9, the internal reliability (Cronbach's $\alpha=0.89$ ) and test-retest reliability $(r=0.84)$ of the PHQ-8 has been shown to be high. ${ }^{134}$ The PHQ-8 consists of eight criteria for the identification and diagnosis of clinically significant depression. These criteria included: loss of pleasure, depressed mood, sleep problems, little energy, change in appetite, feeling of failure, difficulty concentrating, feeling slow or agitated. For analytic purposes, the responses to depression and anxiety questions were scored as $0=0$ days, $1=1-5$ days, $2=6-10$ days, $3=11-14$ days as shown previously. ${ }^{135}$ Item scores were summed for a total score of 0-24. Based on the summed score, current depression was defined as PHQ- $8 \geq 10$, which has an $88 \%$ sensitivity and specificity for moderate depression, ${ }^{134}$ resulting in a dichotomous measures of no 
current depression (score less than 10) and current depression (score $\geq 10$ ). Questions asking about a diagnosis of depression and/or anxiety included: (1) "Has a doctor or other healthcare provider EVER told you that you have depressive disorder (including depression, major depression, dysthymia, or minor depression)?" (2) "Has a doctor or other healthcare provider EVER told you that you had an anxiety disorder (including acute stress disorder, anxiety, generalized anxiety disorder, obsessive-compulsive disorder, panic disorder, phobia, PTSD, or social anxiety disorder?" Response options included yes/no.

The 17-item self-report Deliberate Self-Harm Inventory (DSHI) was used to measure selfinjurious behavior. ${ }^{62}$ A review of the psychometric properties of the DSHI scale reported good internal consistency (Cronbach's $\alpha=.81-.96)$ and test-retest reliability $(r=.65-.91) .{ }^{62}$ The DSHI has been tested in both community and clinical samples. ${ }^{62,136}$ Participants who answered "yes" to any type of deliberate self-harm were categorized as positive and those who answered "no" to all DSHI questions were categorized as negative deliberate self-harm behavior.

A four-item modified Suicide Behaviors Questionnaire (SBQ) was used to measure suicidal thoughts and behaviors. ${ }^{137}$ The modified 4-item SBQ has shown satisfactory reliability and validity in clinical and non-clinical populations. ${ }^{138,139}$ The SBQ instrument has been tested among college populations and demonstrated good internal consistency among non-clinical (Cronbach's $\alpha=0.80$ ) and clinical (Cronbach's $\alpha=0.75$ ), and test-retest correlations also were good $(\mathrm{r}=0.95) .{ }^{138,140,141}$ The SBQ item-1 (past suicidal thoughts or attempts; "Have you ever thought about or attempted to commit suicide?") was rated on a 4-point scale (1= never, 4= I have attempted to kill myself, and really hoped to die). The SBQ item-2 (frequency of suicide ideation in lifetime; "How often have you thought about killing yourself in your lifetime?") was rated on 5-point scale ( $1=$ never, 5= very often). The SBQ item-3 (past suicide thoughts, "Have 
you ever thought about or attempted to kill in your lifetime?") was rated on a 3 -point scale (1= no, 3= yes, more than one period of time). The SBQ item-4 (likelihood of suicide attempt, "How likely that you will attempt suicide in your lifetime?") was rated on 6-point scale (1= no chance at all, $6=$ great chance). The responses to these four items were summed and a cutoff score of 7 was used to assign participants into one of the two groups: suicide behavior group (score of 7 or higher) and no suicide behavior group (score of 6 or lower).

Interpersonal violence and intimate partner violence items measured perpetration of physical violence and psychological aggression within peer relationships and dating relationships. These items were adapted from Youth Violence Survey: Linkages among Different forms of Violence. ${ }^{142}$ These measures have been previously used for both young males and females and have shown high reliability with Cronbach's alphas ranging from 0.84 to $0.92 .{ }^{142}$ The perpetration of interpersonal and intimate partner violence was categorized into three major types: physical violence, emotional violence, and sexual violence, as shown previously. ${ }^{142}$

\subsubsection{Data Analysis}

Chi-Square test was used to identify significant differences between frequencies, and for those variables where a cell count was $\leq 10$, a Fisher's exact test was used. Initial analyses using unadjusted bivariate logistic regression models were conducted to identify relationships between sexual victimization and each independent variable. We first examined the unadjusted odds ratios and 95\% confidence intervals (CI) for any association between dependent and independent variables. Multivariate logistic regression models were calculated controlling for age, relationship status, race/ethnicity, and year in school as potential confounders. All frequencies and regression models were calculated using SAS (Ver. 9.1). 


\subsection{Results}

3.3.1 Prevalence of Risk Behaviors, Negative Health Outcomes, and Sexual Victimization among College-attending Males and Females

Demographic information for the analytic sample is provided in Table 3.1. A majority of the participants were between the ages of $18-19$ years $(64.72 \%)$, white $(92.50 \%)$, single $(61.53 \%)$, heterosexual (96.39\%), and were not a member of a fraternity or sorority (91.49\%).

Demographic characteristics were similar for both male and female participants. The prevalence of risk behaviors among male and female participants is provided in Table 3.2. When compared with females, male participants were more likely to use tobacco $\left(41.24 \%, \chi^{2}=17.98\right.$, p-value $<0.05)$, marijuana $\left(43.88 \%, \chi^{2}=5.07, p\right.$-value $\left.<0.05\right)$, drugs other than cocaine $\left(10.65 \%, \chi^{2}=\right.$ 4.31, p-value $<0.05)$, and report heavy episodic drinking $\left(30.37 \%, \chi^{2}=6.20\right.$, p-value $\left.<0.05\right)$ in the past 30 days.

The prevalence of different categories of sexual victimization was higher among female participants when compared with males (Table 3.3). Overall, females reported more unwanted sexual contact $\left(47.72 \%, \chi^{2}=32.06\right.$, p-value $\left.<0.05\right)$, sexual coercion $\left(35.53 \%, \chi^{2}=24.81, \mathrm{p}-\right.$ value $<0.05)$, attempted rape $\left(35.64, \chi^{2}=28.33\right.$, p-value $\left.<0.05\right)$, sexual assault with an object $\left(31.45, \chi^{2}=5.51, p\right.$-value $\left.<0.05\right)$, and completed rape $\left(39.83 \%, \chi^{2}=6.79\right.$, p-value $\left.<0.05\right)$ as compared with males. No significant differences were observed in the prevalence of sexual victimization due to drugs or alcohol given by perpetrator, drug or alcohol used independent of partner use, or coercion.

Table 3.4A shows the prevalence of various negative health outcomes and risk behaviors among males. No significant differences were observed among victimized and non-victimized males for poor self-reported health status, poor mental health, low life satisfaction, or activity limitations. 
However, victimized males, except for those who experienced attempted rape, showed a significantly higher prevalence of current depression (sexual contact: $28.57 \%, \chi^{2}=8.72$, p-value $<0.05$; coercion: $38.10 \%$, p-value $<0.05$; sexual assault with an object: $26.83 \%, \chi^{2}=7.87$, pvalue $<0.05$; completed rape: $27.66 \%, \chi^{2}=13.19$, p-value $<0.05$; drug or alcohol give by perpetrator: $45.16 \%$, p-value $<0.05$; drug and alcohol used willingly: $21.25 \%, \chi^{2}=5.70$, p-value $<0.05$; external pressure: $30.95, \chi^{2}=11.92$, $\mathrm{p}$-value $<0.05$ ) when compared with non-victimized males. Prevalence of tobacco use was higher among males who experienced sexual assault with an object $\left(63.41 \%, \chi^{2}=11.21\right.$, p-value $\left.<0.05\right)$, completed rape $\left(59.57 \%, \chi^{2}=9.80\right.$, p-value $<0.05)$, and unwanted sexual experience after consuming alcohol and drugs $\left(56.25 \%, \chi^{2}=12.42\right.$, p-value $<0.05$ ). Similarly, alcohol and marijuana use in the past 30 days was also higher among males who experienced sexual assault with an object (alcohol: $100 \%, \chi^{2}=10.69$, p-value $<0.05$; marijuana: $65.38 \%, \chi^{2}=6.31$, p-value $<0.05$ ), completed rape (alcohol: $96.55 \%, \chi^{2}=7.64$, pvalue $<0.05$; marijuana: $61.29 \%, \chi^{2}=4.14$, p-value $<0.05$ ), and unwanted sexual experience after consuming alcohol and drugs (alcohol: $98.04 \%, \chi^{2}=18.51$, p-value $<0.05$; marijuana: $100 \%, \chi^{2}=6.96, p$-value $\left.<0.05\right)$. Even though the prevalence of cocaine use was lower in the total male sample, a higher percentage of victimized males (with the exceptions of sexual contact and completed rape) reported cocaine use in the last 30 days when compared with nonvictimized males. A higher percentage of heavy episodic drinking (five or more alcohol drinks at one occasion) was reported by some categories of victimized males (sexual assault with an object: $51.22 \%, \chi^{2}=11.77$, p-value $<0.05$; completed rape: $48.94 \%, \chi^{2}=8.82$, p-value $<0.05$, drugs or alcohol given by yourself: $51.61, \chi^{2}=6.15$, p-value $<0.05$; drug and alcohol use by yourself: $48.10 \%, \chi^{2}=17.46$ ), p-value $<0.05$ ) when compared with non-victimized males. 
The prevalence of negative health outcomes and risk behaviors among victimized and nonvictimized female participants are shown in Table 3.4B. A higher percentage of females who had experienced unwanted sexual contact $\left(49.17 \%, \chi^{2}=8.23\right.$, p-value $\left.<0.05\right)$ and completed rape $\left(50.52 \%, \chi^{2}=9.34, p\right.$-value $\left.<0.05\right)$ reported 6 or more days with poor mental health when compared with non-victimized females. A higher prevalence of activity limitations were reported by females who experienced sexual coercion $\left(54.66 \%, \chi^{2}=4.87\right.$, p-value $\left.<0.05\right)$, attempted rape $\left(55.17 \%, \chi^{2}=7.75\right.$, p-value $\left.<0.05\right)$, completed rape $\left(53.40 \%, \chi^{2}=6.57\right.$, p-value $<0.05)$, and unwanted sexual experience when consumed drugs or alcohol $\left(52.33 \%, \chi^{2}=7.84\right.$, pvalue $<0.05)$ when compared with non-victimized females. Similar to males, the prevalence of current depression was also higher among all victimized females when compared with nonvictimized females. A higher percentage of women who experienced sexual victimization (with the exception of drug and alcohol use and external pressure) also reported being diagnosed with depression and anxiety disorder. Tobacco use in the past 30 days was also higher among women who experienced attempted rape $\left(32.0 \%, \chi^{2}=4.87\right.$, $\mathrm{p}$-value $\left.<0.05\right)$, sexual assault with an object $\left(38.75 \%, \chi^{2}=24.72\right.$, p-value $\left.<0.05\right)$, completed rape $\left(37.70 \%, \chi^{2}=24.46\right.$, p-value $\left.<0.05\right)$, unwanted sexual experience when perpetrator gave drugs or alcohol $\left(38.46 \%, \chi^{2}=13.68\right.$, pvalue $<0.05$ ), and unwanted sexual experience when drugs and alcohol were consumed willingly $\left(35.80 \%, \chi^{2}=31.50, \mathrm{p}\right.$-value $\left.<0.05\right)$. Similarly, a higher percentage of females who experienced unwanted sexual contact $\left(85.84 \%, \chi^{2}=14.21\right.$, p-value $\left.<0.05\right)$, attempted rape $\left(87.95 \%, \chi^{2}=\right.$ 10.66, p-value $<0.05)$, sexual assault with an object $\left(95.40 \%, \chi^{2}=31.52\right.$, p-value $\left.<0.05\right)$, completed rape $\left(37.39 \%, \chi^{2}=24.46\right.$, p-value $\left.<0.05\right)$, unwanted sexual experience when perpetrator gave drugs or alcohol $\left(17.41 \%, \chi^{2}=13.68\right.$, p-value $\left.<0.05\right)$, and unwanted sexual experience when consumed drugs and alcohol willingly $\left(67.23 \%, \chi^{2}=31.50\right.$, p-value $\left.<0.05\right)$ 
reported alcohol use when compared with non-victimized females. All victimized females, except for those who experienced unwanted sexual activity due to external pressure, reported higher use of marijuana when compared with non-victimized females. The use of cocaine in the past 30 days was higher among female participants who experienced completed rape $\left(5.49 \%, \chi^{2}\right.$ $=4.60, \mathrm{p}$-value $<0.05)$ when compared with females who did not experience any victimization. Episodic drinking in the past 30 days was also higher among victimized females (except for those who experience sexual coercion) when compared with non-victimized females.

\subsubsection{Associations between Aggressive Behaviors and Sexual Victimization among Males}

The prevalence of aggressive behaviors among victimized and non-victimized male participants is shown in Table 3.5. A higher percentage of interpersonal physical violence was reported by men who experienced sexual assault with an object $\left(68.29 \%, \chi^{2}=6.45, \mathrm{p}\right.$-value $\left.<0.05\right)$, completed rape $\left(70.21 \%, \chi^{2}=6.74\right.$, p-value $\left.<0.05\right)$, unwanted sexual experience when drugs or alcohol were given by the perpetrator $\left(70.97 \%, \chi^{2}=4.47, p\right.$-value $\left.<0.05\right)$, and unwanted sexual experience as a result of external pressure $\left(69.05 \%, \chi^{2}=6.21\right.$, p-value $\left.<0.05\right)$ when compared with non-victimized males. The perpetration of interpersonal emotional violence was higher among males who experienced sexual assault with an object $\left(78.95 \%, \chi^{2}=5.58, p\right.$-value $\left.<0.05\right)$ and unwanted sexual activity when the perpetrator gave drugs or alcohol $\left(83.87 \%, \chi^{2}=5.57, \mathrm{p}\right.$ value $<0.05$ ) when compared with males who did not report any victimization. When compared with non-victimized males, the perpetration of interpersonal sexual violence was significantly higher among all victimized males except for those who experienced unwanted sexual contact and unwanted sexual activity when the perpetrator gave drugs or alcohol. The prevalence of intimate partner physical violence was significantly higher among males who experienced unwanted sexual contact $(28.21 \%$, p-value $<0.05)$, sexual coercion $(42.86 \%$, p-value $<0.05)$, 
attempted rape (34.78\%, p-value<0.05), and unwanted sexual activity when the perpetrator gave drugs and alcohol $\left(23.33 \%, \chi^{2}=5.99, \mathrm{p}\right.$-value $\left.<0.05\right)$ when compared with non-victimized males. Additionally, when compared with non-victimized males, the prevalence of intimate partner emotional violence was higher among males who reported attempted rape $\left(82.91 \%, \chi^{2}=\right.$ 4.24, p-value <0.05), sexual assault with an object $\left(80.49 \%, \chi^{2}=6.64\right.$, p-value $\left.<0.05\right)$, completed rape $\left(80.85 \%, \chi^{2}=8.75\right.$, p-value $\left.<0.05\right)$, unwanted sexual experience when the perpetrator gave alcohol $\left(80.65 \%, \chi^{2}=4.84\right.$, p-value $\left.<0.05\right)$, and unwanted sexual activity after willingly consuming drugs or alcohol $\left(75.95 \%, \chi^{2}=9.82\right.$, p-value $\left.<0.05\right)$.

Table 3.6 presents the unadjusted odds ratios (OR) of the relationship between aggressive behaviors and different types of sexual victimization. Our results show that men who had experienced sexual assault with an object, completed rape, and unwanted sexual experience when perpetrator gave drugs or alcohol were more likely to perpetrate interpersonal physical violence when compared with non-victimized males. The odds of perpetration of interpersonal emotional violence were also higher among males who experienced sexual assault with an object $(\mathrm{OR}=2.60,95 \% \mathrm{CI}=1.15,5.85)$ and unwanted sexual experience when the perpetrator gave them drugs or alcohol $(\mathrm{OR}=3.25,95 \%=1.17,8.96)$. Men who reported sexual coercion, attempted rape, sexual assault with an object, completed rape, unwanted sexual activity after consuming drugs and alcohol, and unwanted experience due to external pressure had higher odds of perpetration of interpersonal sexual violence when compared with non-victimized males.

Unadjusted odds ratios for intimate partner physical violence were higher among males who experienced unwanted sexual contact $(\mathrm{OR}=4.87,95 \% \mathrm{CI}=1.88,12.59)$, sexual coercion $(\mathrm{OR}=9.83,95 \% \mathrm{CI}=3.27,29.49)$, attempted rape $(\mathrm{OR}=7.82,95 \% \mathrm{CI}=2.62,23.30)$, and unwanted 
sexual activity when the perpetrator provided drugs or alcohol $(\mathrm{OR}=3.48,95 \% \mathrm{CI}=1.22,9.92)$ when compared with non-victimized males. With the exception of males who reported unwanted sexual contact, sexual coercion, and unwanted sexual experience due to external pressure, the odds of intimate partner emotional violence perpetration were higher among victimized males when compared with non-victimized males. The odds of perpetration of intimate partner sexual violence were also higher among males who reported unwanted sexual contact $(\mathrm{OR}=3.25,95 \%$ $\mathrm{CI}=1.37,7.70)$, attempted rape $(\mathrm{OR}=3.19,95 \% \mathrm{CI}=1.14,8.87)$, sexual assault with an object $(\mathrm{OR}=3.00,95 \% \mathrm{CI}=1.25,7.20)$, and unwanted sexual experience due to external pressure $(\mathrm{OR}=2.61,95 \% \mathrm{CI}=1.10,6.20)$.

The adjusted odds ratios $\left(\mathrm{OR}_{\mathrm{adj}}\right)$ for the relationships between aggressive behaviors and different types of sexual victimization are shown in Table 3.7. The multivariate models were adjusted for age, race, year in school, and current relationship status. After adjusting for these covariates, our results show that when compared to non-victimized males, men who experienced sexual assault with an object, completed rape, and unwanted sexual experience when perpetrator gave drugs or alcohol were more likely to perpetrate interpersonal physical violence. For victimized males who had experienced a sexual assault with an object and unwanted sexual experience when the perpetrator provided drugs or alcohol, the odds of interpersonal emotional violence were 2.57 $(95 \% \mathrm{CI}=1.12,5.89)$ and $3.35(95 \% \mathrm{CI}=1.15,9.72)$ greater, respectively, when compared with non-victimized males.

The multivariate models also show that men who reported sexual coercion, attempted rape, sexual assault with an object, completed rape, unwanted sexual activity after consuming drugs and alcohol, and unwanted experience due to external pressure had higher odds of perpetration of interpersonal sexual violence when compared with non-victimized males. In addition to 
unwanted sexual contact, sexual coercion, attempted rape, and unwanted sexual experience when the perpetrator provided drugs or alcohol, the odds of intimate partner physical violence were also greater for males who had experienced sexual assault with an object $\left(\mathrm{OR}_{\mathrm{adj}}=3.07,95 \%\right.$ $\mathrm{CI}=1.10,8.51)$ when compared with non-victimized males. This association was not observed in the unadjusted models. In comparison to non-victimized males, the odds of perpetration of intimate partner emotional violence were also greater among males who had experienced attempted rape $\left(\mathrm{OR}_{\mathrm{adj}}=3.52,95 \% \mathrm{CI}=1.07,11.53\right)$, sexual assault with an object $\left(\mathrm{OR}_{\mathrm{adj}}=2.97\right.$, $95 \% \mathrm{CI}=1.25,7.07)$, completed rape $\left(\mathrm{OR}_{\mathrm{adj}}=3.27,95 \% \mathrm{CI}=1.41,7.55\right)$, unwanted sexual activity when perpetrator provided drugs or alcohol $\left(\mathrm{OR}_{\mathrm{adj}}=3.01,95 \% \mathrm{CI}=1.11,8.17\right)$, and unwanted sexual activity after consuming drugs or alcohol willingly $\left(\mathrm{OR}_{\mathrm{adj}}=3.01,95 \% \mathrm{CI}=1.50,6.04\right)$. The odds of perpetration of intimate partner sexual violence were also higher among all victimized males, except for those who experience completed rape, as compared with nonvictimized males.

\subsubsection{Associations between Suicidal Behavior, DSH, and Sexual Violence among Victimized Males and Females}

We also compared the prevalence of suicidal behaviors and deliberate self-harm (DSH) among victimized males and females (Table 3.8). Overall, there were few statistically significant differences in the prevalence of suicidal behaviors between these groups. The only significant difference in the prevalence of DSH was observed among victimized males (80.95\%) and females $(58.75 \%)$ who experienced sexual coercion. We did not observe any statistically significant differences in the prevalence of DSH among males and females who experienced other types of sexual victimization. 
The unadjusted and adjusted odds ratios for relationships between sexual victimization and suicidal behaviors and DSH are shown in Tables 3.9 and 3.10, respectively. While the odds of suicidal behaviors were higher among victimized males when compared with non-victimized females, no significant associations were observed. Similarly, odds of DSH were relatively lower among victimized males when compared with non-victimized females; no significant associations were identified.

\subsection{Discussion}

In this study we examined gender differences in multiple categories of sexual victimization and the associations between victimization and adverse mental health outcomes among college-aged students. Included in this study were detailed considerations of the prevalence of substance use (tobacco, alcohol, cocaine, episodic drinking), negative health outcomes (current health status, mental health, life satisfaction, activity limitation, current depression), aggressive behaviors (interpersonal and intimate partner violence), and adverse psychological consequences (suicidal behaviors and DSH).

Our results showed a higher prevalence of risk behavior, such as tobacco, alcohol, cocaine use and episodic drinking, among male participants when compared with females. However, the prevalence of tobacco and alcohol use (episodic and non-episodic) among male and female participants was comparable to those reported previously among samples of similar populations. ${ }^{143-145}$ Consistent with results from previous studies, more female participants (victimized and non-victimized) reported outcomes as such poor mental health, life satisfaction, and activity limitations than males. ${ }^{126,146}$ 
Our results suggest that both men and women reported unwanted sexual experiences. As anticipated, a higher prevalence of sexual victimization was reported by female participants than males. The prevalence of some types of sexual victimization (sexual contact, sexual coercion, attempted rape, alcohol-related sexual victimization) among females was comparable to those previously reported where similar definitions of sexual victimization have been used. ${ }^{147-149}$ Previous studies have also estimated the prevalence of sexual victimization among collegeattending males. ${ }^{5,92,150}$ Larimar et al. (1999) reported prevalence rates for unwanted sexual experience among male and female college students using the same SES measure used in this study. ${ }^{92}$ We observed a similar pattern of gender differences in outcomes in our sample when compared to findings of previous studies (i.e. higher prevalence of unwanted sexual experience among female students when compared with males). However, the study by Larimar et al. (2004) was limited to those who were members of a fraternity or sorority at the time of the study. Similar to previous findings, a large number of male and female participants in our study reported engaging in unwanted sexual activity as a result of their use of alcohol or when the perpetrator provided alcohol prior to the assault. ${ }^{3,151}$ Alcohol has been identified as an important and modifiable risk factor commonly associated with sexual victimization among college students. ${ }^{3,85,152}$ Previous studies have suggested that the prevalence of alcohol-related sexual assault may be as high as $50 \%$ among college students., ${ }^{3,92}$ Studies examining alcohol-related sexual victimization have reported that alcohol was often consumed by both perpetrators and victims. Although the prevalence of alcohol-related sexual assault among women has been wellstudied, comparatively fewer studies have examined the role of alcohol in male sexual assault. Findings from our study suggest that there is a need to examine the gender difference in alcoholrelated sexual assaults in greater detail. 
One of the objectives of this study was to examine reports of interpersonal and intimate partner violence perpetration among males who had experienced sexual victimization. To our knowledge, this is one of the first studies to examine the association between aggressive behaviors and sexual violence among victimized college-attending males. We observed significantly higher perpetration of physical, emotional, and sexual violence by victimized males when compared with non-victimized males. Significant associations between perpetration of aggressive behavior and a history of victimization, especially among those who had experienced more severe victimization such as attempted rape, sexual assault with an object, and completed rape, were observed. Previous studies have not measured the perpetration of violence behavior among victimization males, but suggest that victimized males may be more likely to report increases in aggression and other externalized behaviors. ${ }^{6}$ Victimized males may commonly blame themselves for not stopping the assault, question their roles in the assault, and may report more hostility and anger. ${ }^{6}$ Other studies have suggested that the long-term impacts of sexual assault on males may result in increased expressions of anger, hostility, and aggression, which have been associated with an increased risk of suicidal or violent behaviors. ${ }^{7,20}$

Previous studies have suggested that similar to women, men who were raped as adults also experience post-traumatic stress disorder, depression, anxiety, anger, self-blame, and emotional distancing. ${ }^{7,21,42-44}$ Results from the current study identified no significant difference in suicidal behaviors among victimized males and females. However, we did observe current depression among almost all of the victimized males and females, and depression is often associated with suicide ideation/attempts among victimized males. ${ }^{7,18,48}$ A recent review of literature on male sexual assault reported that suicide attempts are more common among adolescent and young 
adult victims. ${ }^{12}$ We did not identify any significant differences in suicidal behaviors among male and female participants.

Similar to findings on suicidal behaviors, no significant differences in deliberate self-harm were observed. The prevalence of DSH was lower in the overall sample and victimized sample. Therefore, it is possible that the failure to identify gender differences may be due to lack of statistical power and small size of our victimized samples. It is also possible that the associations between sexual assault and self-inflicted injury may not be immediately observable and may increase in intensity over time. These findings suggest that more definitive research on emotional consequences of unwanted sexual experience using longitudinal data is needed.

Results from this study are subject to several limitations. First, the demographic composition of our sample appears to be representative of the departmental composition of the university where the study was conducted. However, it may not be representative of the overall student population in either the university at-large or of students in other areas, thus limiting the generalizability of our findings. Second, the participants who choose to participate in the study may have had a particular interest in the topic, thus inviting selection bias. Also, recall bias may have occurred, as information on sexual victimization comes from self-reported data. Also, the data are cross-sectional rather than longitudinal, therefore no causal relationships can be drawn from these findings. Additionally, these findings are based on self-reports of the data and may not provide an accurate estimate of the prevalence of sexual victimization.

This study has provided data on the prevalence and correlates of unwanted sexual experience among male and female college students attending a large Mid-Atlantic university. Our findings confirm the importance of identifying gender-specific differences in the prevalence of sexual 
victimization and understanding its psychological impact on both males and females independently. Sexual victimization has been associated with severe negative consequences, and it is important to have a clear understanding of its impact on the physical and mental health of young males and females. We observed significant associations between sexual victimization and perpetration of interpersonal and intimate partner physical, sexual and emotional violence.

The findings emphasize the need for better understanding of male sexual assault, especially among young adults. Further empirical study of the emotional consequences of unwanted sexual experience for both genders is warranted. Given the evidence that men can be victims of sexual assault and that these unwanted experiences can occur on college campuses, a number of intervention strategies can be implemented by universities in an effort to reduce the rate and consequences of sexual assault. As suggested above, the adverse consequences of sexual assault may contribute to an inability to manage interpersonal relationships. The development of interpersonal and intimate relationships is of critical importance for young adults and the psychological impact of sexual assault on these relationships serves as a reminder of the necessity of effective prevention and early intervention. While most prevention programs on college campuses focus on risk for female students, results from this research project, in concert with results from existing studies, highlight the need for programs to consider both male and female victims of sexual assault. 
Table 3.1: Demographic Characteristics of Study Population

\begin{tabular}{|c|c|c|c|}
\hline Variable & $\begin{array}{c}\text { Male } \quad(N=194) \\
n \text { (Percentage) }\end{array}$ & $\begin{array}{c}\text { Female } \quad(N=526) \\
n(\text { Percentage })\end{array}$ & $\begin{array}{c}\text { Total } \quad(N=720) \\
n(\text { Percentage })\end{array}$ \\
\hline \multicolumn{4}{|l|}{ Age Groups (in years) } \\
\hline $18-19$ & $116(59.69)$ & $350(66.54)$ & $466(64.72)$ \\
\hline $20-21$ & $57(29.38)$ & $141(26.81)$ & $198(27.50)$ \\
\hline $22-23$ & $13(6.70)$ & $17(3.23)$ & $30(4.17)$ \\
\hline 24-25 & $3(1.55)$ & $12(2.28)$ & $15(2.08)$ \\
\hline $25+$ & $5(2.58)$ & $6(1.14)$ & $11(1.53)$ \\
\hline \multicolumn{4}{|l|}{ Year in School } \\
\hline $1^{\text {st }}$ Year & $94(48.45)$ & $263(50.00)$ & $357(49.58)$ \\
\hline $2^{\text {nd }}$ Year & $49(25.26)$ & $140(26.62)$ & $189(26.25)$ \\
\hline $3^{\text {rd }}$ Year & $31(15.98)$ & $67(12.74)$ & $98(13.61)$ \\
\hline $4^{\text {th }}$ Year & $13(6.70)$ & $35(6.65)$ & $48(6.67)$ \\
\hline $5^{\text {th }}$ Year & $3(1.55)$ & $16(3.04)$ & $19(2.64)$ \\
\hline Graduate & $1(0.52)$ & 1() $.19)$ & $2(0.28)$ \\
\hline Other & $3(1.55)$ & $4(0.76)$ & $7(0.97)$ \\
\hline \multicolumn{4}{|l|}{ Race } \\
\hline White & $179(92.27)$ & $487(92.59)$ & $666(92.50)$ \\
\hline African American & $6(3.09)$ & $14(2.66)$ & $20(2.78)$ \\
\hline Hispanic & $3(1.55)$ & $6(1.14)$ & $9(1.25)$ \\
\hline Asian or Pacific Islander & $2(1.03)$ & $5(0.95)$ & $7(0.97)$ \\
\hline Other & $4(2.06)$ & $14(2.66)$ & $18(2.50)$ \\
\hline \multicolumn{4}{|l|}{ Current Relationship Status } \\
\hline Single & $135(69.95)$ & $308(58.56)$ & $443(61.53)$ \\
\hline Separated/Widowed & $1(0.52)$ & $0(0.0)$ & $1(0.14)$ \\
\hline Married/Cohabitating & $5(2.58)$ & $6(1.14)$ & $11(1.53)$ \\
\hline Divorced & $0(0.0)$ & $2(0.38)$ & $2(0.28)$ \\
\hline Committed & $53(27.32)$ & $210(39.92)$ & $263(36.53)$ \\
\hline \multicolumn{4}{|l|}{ Sexual Orientation } \\
\hline Heterosexual & $188(96.91)$ & $506(96.20)$ & $694(96.39)$ \\
\hline Bisexual & $2(1.03)$ & $11(2.09)$ & $13(1.81)$ \\
\hline Gay/Lesbian & $2(1.03)$ & $4(0.76)$ & $6(0.83)$ \\
\hline Unsure & $2(1.03)$ & $5(0.95)$ & $7(0.97)$ \\
\hline \multicolumn{4}{|c|}{ Member of Fraternity/Sorority } \\
\hline Yes & $19(9.84)$ & $42(8.02)$ & $61(8.51)$ \\
\hline No & $174(90.16)$ & $482(91.98)$ & $656(91.49)$ \\
\hline
\end{tabular}


Table 3.2: Prevalence of Risk Behaviors among Male and Female Participants

\begin{tabular}{|c|c|c|c|c|}
\hline Risk Behaviors & Response & $\begin{array}{c}\text { Males } \\
\text { n (Percentage })\end{array}$ & $\begin{array}{c}\text { Females } \\
n \text { (Percentage) }\end{array}$ & $\begin{array}{c}\text { Total } \\
n(\text { Percentage })\end{array}$ \\
\hline Tobacco use $>5$ days in past 30 days* & No & $114(58.76)$ & $393(75.00)$ & $393(75.00)$ \\
\hline Alcohol use $>5$ days in past 30 days & No & $24(22.22)$ & $64(26.02)$ & $88(2486)$ \\
\hline Marijuana use in past 30 days* & Yes & $61(43.88)$ & $134(33.25)$ & $195(35.98)$ \\
\hline \multirow[t]{2}{*}{ Cocaine use in past 30 days } & Yes & $9(5.11)$ & $14(2.90)$ & $23(3.49)$ \\
\hline & No & $167(94.89)$ & $469(97.10)$ & $636(96.51$ \\
\hline \multirow[t]{2}{*}{ Other drug use in past 30 days* } & Yes & $18(10.65)$ & $28(5.87)$ & $46(7.12)$ \\
\hline & No & $151(89.35)$ & $449(94.13)$ & $600(92.88)$ \\
\hline $\begin{array}{l}\text { Episodic Drinking (> } 5 \text { drinks) in past } 30 \\
\text { days* }\end{array}$ & Yes & $58(30.37)$ & $111(21.39)$ & $169(23.80)$ \\
\hline
\end{tabular}

* significant differences between male and female participants - $\mathrm{p}$-value $\leq 0.05$ 
Table 3.3: Number of Males and Females Experiencing Sexual Victimization, Categorized by Type

\begin{tabular}{lccc}
\hline Type of Victimization & Male & Female & Total \\
& $\boldsymbol{n}($ Percentage) & $\boldsymbol{n}$ (Percentage) & $\boldsymbol{n}$ (Percentage) \\
\hline Sexual Contact* & $42(23.46)$ & $241(47.72)$ & $283(41.37)$ \\
\hline Coercion* & $21(14.00)$ & $162(35.53)$ & $183(30.20)$ \\
\hline Attempted Rape* & $23(13.77)$ & $175(35.64)$ & $198(30.09)$ \\
\hline Sexual Assault (SA) with an Object* & $41(22.28)$ & $161(31.45)$ & $202(29.02)$ \\
\hline Completed Rape* & & $192(39.83)$ & $239(36.94)$ \\
\hline Drug or Alcohol Given by Perpetrator & $47(28.28)$ & $118(23.98)$ & $149(22.51)$ \\
\hline Drug or Alcohol Use by Self & $31(18.24)$ & $259(51.59)$ & $339(50.15)$ \\
\hline External Pressure & & & \\
\hline
\end{tabular}

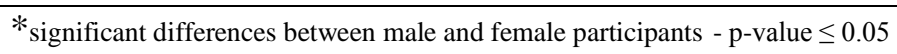


Table 3.4A: Prevalence of Health Outcomes and Risk Behaviors Related to Sexual Victimization among Males ${ }^{\S}$

\begin{tabular}{|c|c|c|c|c|c|c|c|c|c|}
\hline \multirow[b]{2}{*}{ Variables } & & \multicolumn{8}{|c|}{$\begin{array}{c}\text { MALES } \\
n \text { (Percentage) }\end{array}$} \\
\hline & & $\begin{array}{l}\text { Sexual } \\
\text { Contact }\end{array}$ & Coercion & Attempted Rape & $\begin{array}{l}\text { Sexual Assault } \\
\text { (SA) with an } \\
\text { object }\end{array}$ & Completed Rape & $\begin{array}{c}\text { Drug or } \\
\text { Alcohol Given } \\
\text { by Perpetrator }\end{array}$ & $\begin{array}{c}\text { Drug or } \\
\text { Alcohol Use } \\
\text { by yourself }\end{array}$ & $\begin{array}{l}\text { External } \\
\text { Pressure }\end{array}$ \\
\hline \multirow{2}{*}{$\begin{array}{l}\text { Poor self-reported } \\
\text { health status }\end{array}$} & Victimized Male & $0(0)$ & $0(0)$ & $0(0)$ & $0(0)$ & $0(0)$ & $0(0)$ & $0(0)$ & $0(0)$ \\
\hline & Non-Victimized Males & $0(0)$ & $0(0)$ & $0(0)$ & $0(0)$ & $0(0)$ & $0(0)$ & $0(0)$ & $0(0)$ \\
\hline \multirow{2}{*}{$\begin{array}{l}\geq 6 \text { days when mental } \\
\text { health not good in past } \\
30 \text { days }\end{array}$} & Victimized Male & $15(35.71)$ & $8(38.10)$ & $9(39.13)$ & $8(19.51)$ & $11(23.40)$ & $7(22.58)$ & $18(22.50)$ & $16(38.10)$ \\
\hline & Non-Victimized Males & $35(25.55)$ & $34(26.36)$ & 38 (26.39) & $41(28.67)$ & $34(28.81)$ & $39(28.06)$ & $29(30.85)$ & $36(28.28)$ \\
\hline \multirow[t]{2}{*}{ Low life satisfaction } & Victimized Male & 7 (16.67) & $3(14.29)$ & $4(17.39)$ & $4(9.76)$ & $8(17.02)$ & $4(12.90)$ & $11(13.75)$ & $6(14.29)$ \\
\hline & Non-Victimized Males & $11(8.03)$ & $11(8.53)$ & $11(7.64)$ & $13(9.09)$ & $8(6.78)$ & $11(7.91)$ & $5(5.32)$ & $10(7.30)$ \\
\hline \multirow[t]{2}{*}{ Activity limitation } & Victimized Male & $22(52.38)$ & $9(42.86)$ & $11(47.83)$ & $19(46.34)$ & $18(38.30)$ & $17(54.84)$ & $34(42.50)$ & $20(47.62)$ \\
\hline & Non-Victimized Males & $56(41.18)$ & $55(42.64)$ & $60(41.96)$ & $57(40.14)$ & $47(40.17)$ & $55(39.86)$ & $40(43.01)$ & $57(41.91)$ \\
\hline \multirow[t]{2}{*}{ Current depression } & Victimized Male & $12(28.57)$ & 8 (38.10) & $5(21.74)$ & $11(26.83)$ & $13(27.66)$ & $9(29.03)$ & $17(21.25)$ & $13(30.95)$ \\
\hline & Non-Victimized Males & $14(10.22)$ & $14(10.85)$ & $19(13.19)$ & $14(9.79)$ & $8(6.78)$ & $15(10.79)$ & $8(8.51)$ & $13(9.49)$ \\
\hline \multirow{2}{*}{$\begin{array}{l}\text { Diagnosed with } \\
\text { depression disorder }\end{array}$} & Victimized Male & $5(13.89)$ & $4(23.53)$ & $3(16.67)$ & $5(14.29)$ & $5(11.90)$ & $5(17.86)$ & $7(9.72)$ & $4(11.43)$ \\
\hline & Non-Victimized Males & $10(7.87)$ & $9(7.38)$ & $10(7.30)$ & $10(7.52)$ & $8(7.14)$ & $9(6.98)$ & $8(9.09)$ & $11(8.46)$ \\
\hline \multirow{2}{*}{$\begin{array}{l}\text { Diagnosed with anxiety } \\
\text { disorder }\end{array}$} & Victimized Male & $5(14.29)$ & $3(16.67)$ & $2(10.00)$ & $6(15.79)$ & $7(15.56)$ & $5(16.67)$ & $9(12.50)$ & $4(10.53)$ \\
\hline & Non-Victimized Males & $16(12.21)$ & $14(11.29)$ & $15(10.87)$ & $14(10.45)$ & $10(8.85)$ & $14(10.77)$ & $10(11.11)$ & $16(12.31)$ \\
\hline \multirow{2}{*}{$\begin{array}{l}\text { Tobacco use }>5 \text { days in } \\
\text { past } 30 \text { days }\end{array}$} & Victimized Male & $16(38.10)$ & $9(42.86)$ & $10(43.48)$ & $26(63.41)$ & $28(59.57)$ & $17(54.84)$ & $45(56.25)$ & $22(52.38)$ \\
\hline & Non-Victimized Males & $59(43.07)$ & $54(41.86)$ & $57(39.58)$ & $49(34.27)$ & $39(33.05)$ & $53(38.13)$ & $28(29.79)$ & $54(39.42)$ \\
\hline \multirow{2}{*}{$\begin{array}{l}\text { Alcohol use > } 5 \text { days in } \\
\text { past } 30 \text { days }\end{array}$} & Victimized Male & $18(75.00)$ & $11(84.62)$ & $12(92.31)$ & $27(100.00)$ & 28 (96.55) & $22(100)$ & $50(98.04)$ & $22(84.62)$ \\
\hline & Non-Victimized Males & $59(81.94)$ & $58(81.69)$ & $63(79.75)$ & $52(69.33)$ & 45 (71.43) & $57(77.03)$ & $29(64.44)$ & $59(80.82)$ \\
\hline \multirow{2}{*}{$\begin{array}{l}\text { Marijuana use in past } 30 \\
\text { days }\end{array}$} & Victimized Male & $11(36.67)$ & $8(57.14)$ & $9(52.94)$ & $17(65.38)$ & $19(61.29)$ & $14(60.87)$ & $31(60.78)$ & $16(51.61)$ \\
\hline & Non-Victimized Males & $46(47.42)$ & $41(45.05)$ & $46(45.54)$ & $40(38.10)$ & $34(40.00)$ & $42(43.30)$ & $26(36.62)$ & $41(42.71)$ \\
\hline \multirow{2}{*}{$\begin{array}{l}\text { Cocaine use in past } 30 \\
\text { days }\end{array}$} & Victimized Male & $4(11.43)$ & $4(26.67)$ & $5(27.78)$ & $6(18.18)$ & $2(5.41)$ & $4(18.18)$ & $7(10.29)$ & 4 (11.43) \\
\hline & Non-Victimized Males & $5(3.91)$ & $2(1.68)$ & $3(2.26)$ & $1(0.75)$ & $2(1.79)$ & $3(2.27)$ & $1(1.11)$ & $2(1.59)$ \\
\hline \multirow{2}{*}{$\begin{array}{l}\text { Other drug use in past } \\
30 \text { days }\end{array}$} & Victimized Male & $5(15.15)$ & $2(14.29)$ & $5(27.78)$ & $6(17.65)$ & $5(13.51)$ & $5(20.00)$ & $11(16.18)$ & $4(11.76)$ \\
\hline & Non-Victimized Males & $12(9.68)$ & $12(10.62)$ & $11(8.73)$ & $10(7.94)$ & $9(8.65)$ & $10(8.20)$ & $5(6.02)$ & $10(8.33)$ \\
\hline \multirow{2}{*}{$\begin{array}{l}\text { Episodic drinking (>5 } \\
\text { drinks) in past } 30 \text { days }\end{array}$} & Victimized Male & $12(28.57)$ & $7(33.33)$ & $6(26.09)$ & 21 (51.22) & $23(48.94)$ & $16(51.61)$ & $38(48.10)$ & $18(43.90)$ \\
\hline & Non-Victimized Males & $42(31.11)$ & $40(31.50)$ & $47(33.33)$ & $33(23.40)$ & $29(25.00)$ & $39(28.47)$ & 17 (18.28) & $38(28.15)$ \\
\hline
\end{tabular}

§Fisher's exact test was used where cell count was $\leq 10$; Values in bold indicate significant differences between male and female participants - $\mathrm{p}$-value $\leq 0.05$ 
Table 3.4B: Prevalence of Health Outcomes and Risk Behaviors Related to Sexual Victimization among Females ${ }^{\S}$

\begin{tabular}{|c|c|c|c|c|c|c|c|c|c|}
\hline \multirow{2}{*}{ Variables } & & \multicolumn{8}{|c|}{$\begin{array}{c}\text { FEMALES } \\
n(\text { Percentage })\end{array}$} \\
\hline & & Sexual Contact & Coercion & $\begin{array}{c}\text { Attempted } \\
\text { Rape }\end{array}$ & $\begin{array}{c}\text { Sexual Assault (SA) } \\
\text { with an object }\end{array}$ & $\begin{array}{c}\text { Completed } \\
\text { Rape }\end{array}$ & $\begin{array}{c}\text { Drug or } \\
\text { Alcohol Given } \\
\text { by Perpetrator }\end{array}$ & $\begin{array}{c}\text { Drug or } \\
\text { Alcohol Use by } \\
\text { yourself }\end{array}$ & $\begin{array}{l}\text { External } \\
\text { Pressure }\end{array}$ \\
\hline \multirow{2}{*}{$\begin{array}{l}\text { Poor self-reported } \\
\text { health status }\end{array}$} & Victimized Female & $0(0)$ & $0(0)$ & $0(0)$ & $1(0.62)$ & $1(0.52)$ & $0(0)$ & $1(0.39)$ & $0(0)$ \\
\hline & Non-Victimized Female & $0(0)$ & $0(0)$ & $1(0.32)$ & $0(0)$ & $1(0.52)$ & $1(0.27)$ & $0(0)$ & $1(0.26)$ \\
\hline \multirow{2}{*}{$\begin{array}{l}\geq 6 \text { days when mental } \\
\text { health not good in past } \\
\text { 30 days }\end{array}$} & Victimized Female & $118(49.17)$ & $77(47.83)$ & $85(48.85)$ & $71(44.10)$ & $97(50.52)$ & $58(49.15)$ & $118(45.56)$ & 48 (47.06) \\
\hline & Non-Victimized Female & $96(36.50)$ & $123(41.84)$ & $127(40.32)$ & $144(41.26)$ & $105(36.46)$ & 149 (40.05) & $92(38.17)$ & $159(40.77)$ \\
\hline \multirow[t]{2}{*}{ Low life satisfaction } & Victimized Female & $18(7.47)$ & $16(9.88)$ & $15(8.57)$ & $12(7.45)$ & $17(8.85)$ & $10(8.47)$ & $21(8.11)$ & $11(10.68)$ \\
\hline & Non-Victimized Female & $18(6.82)$ & $20(6.80)$ & $18(5.70)$ & $23(6.55)$ & $19(6.55)$ & $24(6.42)$ & $15(6.17)$ & $24(6.14)$ \\
\hline \multirow[t]{2}{*}{ Activity limitation } & Victimized Female & $118(49.37)$ & 88 (54.66) & $96(55.17)$ & $82(51.25)$ & $102(53.40)$ & $62(52.99)$ & $135(52.33)$ & $54(52.43)$ \\
\hline & Non-Victimized Female & $117(44.83)$ & $128(43.84)$ & $132(42.04)$ & $157(45.24)$ & 119 (41.46) & $164(44.32)$ & $95(39.75)$ & $175(45.34)$ \\
\hline \multirow[t]{2}{*}{ Current depression } & Victimized Female & $62(25.73)$ & $51(31.48)$ & $47(26.86)$ & $46(28.57)$ & $55(28.65)$ & $38(32.20)$ & $74(28.57)$ & $34(33.01)$ \\
\hline & Non-Victimized Female & 48 (18.18) & $52(17.69)$ & $59(18.67)$ & $65(18.52)$ & $48(16.55)$ & $70(18.72)$ & $33(13.58)$ & $74(18.93)$ \\
\hline \multirow{2}{*}{$\begin{array}{l}\text { Diagnosed with } \\
\text { depression disorder }\end{array}$} & Victimized Female & $52(22.03)$ & $42(26.58)$ & $42(24.56)$ & 38 (24.36) & $44(23.53)$ & $29(25.44)$ & $52(20.39)$ & $19(18.81)$ \\
\hline & Non-Victimized Female & 33 (12.99) & $39(13.68)$ & $41(13.36)$ & 48 (14.08) & $36(12.77)$ & $54(14.88)$ & $35(15.09)$ & $63(16.67)$ \\
\hline \multirow{2}{*}{$\begin{array}{l}\text { Diagnosed with anxiety } \\
\text { disorder }\end{array}$} & Victimized Female & $53(22.84)$ & $41(26.28)$ & $43(25.44)$ & $39(25.32)$ & $46(24.73)$ & $30(26.55)$ & $51(20.48)$ & $21(21.21)$ \\
\hline & Non-Victimized Female & 39 (15.35) & $46(16.14)$ & $47(15.41)$ & $56(16.62)$ & $44(15.71)$ & $58(16.20)$ & $43(18.53)$ & $68(18.13)$ \\
\hline \multirow{2}{*}{$\begin{array}{l}\text { Tobacco use }>5 \text { days } \\
\text { in past } 30 \text { days }\end{array}$} & Victimized Female & $63(26.25)$ & $47(29.01)$ & $56(32.00)$ & $62(38.75)$ & $72(37.70)$ & $45(38.46)$ & $92(35.80)$ & $30(29.13)$ \\
\hline & Non-Victimized Female & $65(24.71)$ & $70(23.89)$ & $72(22.86)$ & $64(18.29)$ & $51(17.59)$ & 80 (21.39) & 34 (13.99) & $96(24.62)$ \\
\hline \multirow{2}{*}{$\begin{array}{l}\text { Alcohol use > } 5 \text { days in } \\
\text { past } 30 \text { days }\end{array}$} & Victimized Female & 97 (85.84) & $58(81.69)$ & $73(87.95)$ & $83(95.40)$ & 97 (94.17) & $55(96.49)$ & $131(95.62)$ & $48(84.21)$ \\
\hline & Non-Victimized Female & $80(64.52)$ & 105 (73.94) & $101(68.71)$ & $93(62.42)$ & $71(58.68)$ & $117(69.23)$ & $48(49.48)$ & $125(72.25)$ \\
\hline \multirow{2}{*}{$\begin{array}{l}\text { Marijuana use in past } \\
30 \text { days }\end{array}$} & Victimized Female & $74(40.88)$ & 55 (46.22) & $57(45.60)$ & $60(54.05)$ & $72(53.73)$ & $44(58.67)$ & $91(51.70)$ & $31(42.47)$ \\
\hline & Non-Victimized Female & $56(27.32)$ & $64(28.44)$ & $69(27.71)$ & $70(24.91)$ & $52(22.41)$ & $82(27.33)$ & 39 (18.75) & $96(31.68)$ \\
\hline \multirow{2}{*}{$\begin{array}{l}\text { Cocaine use in past } 30 \\
\text { days }\end{array}$} & Victimized Female & $8(3.69)$ & $6(4.17)$ & $6(3.90)$ & $6(4.35)$ & $9(5.49)$ & $5(4.85)$ & $9(3.98)$ & $4(4.35)$ \\
\hline & Non-Victimized Female & $6(2.43)$ & $7(2.56)$ & $7(2.36)$ & $6(1.81)$ & $5(1.79)$ & $8(2.29)$ & $4(1.71)$ & $9(2.50)$ \\
\hline \multirow{2}{*}{$\begin{array}{l}\text { Other drug use in past } \\
30 \text { days }\end{array}$} & Victimized Female & $17(8.06)$ & $12(8.63)$ & $15(9.93)$ & $15(11.11)$ & $17(10.63)$ & $14(14.00)$ & $21(9.42)$ & $10(11.24)$ \\
\hline & Non-Victimized Female & $10(4.05)$ & $15(5.54)$ & $10(3.42)$ & $11(3.35)$ & $9(3.36)$ & $11(3.20)$ & $5(2.16)$ & 17 (4.78) \\
\hline \multirow{2}{*}{$\begin{array}{l}\text { Episodic drinking (>5 } \\
\text { drinks) in past } 30 \text { days }\end{array}$} & Victimized Female & $65(27.20)$ & $42(26.09)$ & $57(32.76)$ & $63(39.13)$ & $71(37.37)$ & $43(37.39)$ & $90(35.02)$ & $30(29.70)$ \\
\hline & Non-Victimized Female & 44 (16.99) & $61(21.03)$ & $50(16.08)$ & $46(13.37)$ & $30(10.53)$ & $59(15.95)$ & 19 (7.98) & $78(20.21)$ \\
\hline
\end{tabular}


Table 3.5: Prevalence of Aggressive Behaviors among Male Participants ${ }^{\S}$

\begin{tabular}{|c|c|c|c|c|c|c|c|c|c|}
\hline \multirow[b]{2}{*}{ Variables } & & \multicolumn{8}{|c|}{$\begin{array}{c}\text { Types of Victimization } \\
n \text { (Percentage) }\end{array}$} \\
\hline & & Sexual Contact & Coercion & $\begin{array}{l}\text { Attempted } \\
\text { Rape }\end{array}$ & $\begin{array}{l}\text { Sexual Assault } \\
\text { (SA) with an } \\
\text { object }\end{array}$ & $\begin{array}{l}\text { Completed } \\
\text { Rape }\end{array}$ & $\begin{array}{c}\text { Drug or Alcohol } \\
\text { Given by } \\
\text { Perpetrator }\end{array}$ & $\begin{array}{c}\text { Drug or } \\
\text { Alcohol Use by } \\
\text { yourself }\end{array}$ & $\begin{array}{l}\text { External } \\
\text { Pressure }\end{array}$ \\
\hline \multirow{2}{*}{$\begin{array}{l}\text { Perpetrated interpersonal } \\
\text { physical violence }\end{array}$} & Victimized Male & $24(58.54)$ & $15(71.43)$ & $16(69.57)$ & $28(68.29)$ & $33(70.21)$ & $22(70.97)$ & $47(58.75)$ & $29(69.05)$ \\
\hline & Non-Victimized Males & $70(51.47)$ & $67(52.34)$ & $74(51.75)$ & $65(45.77)$ & $56(47.86)$ & $69(50.00)$ & $42(45.16)$ & $64(47.06)$ \\
\hline \multirow{2}{*}{$\begin{array}{l}\text { Perpetrated interpersonal } \\
\text { emotional violence }\end{array}$} & Victimized Male & $30(73.17)$ & $18(85.71)$ & $18(78.26)$ & 32 (78.95) & 35 (74.47) & $26(83.87)$ & $55(68.75)$ & $32(76.19)$ \\
\hline & Non-Victimized Males & $85(62.50)$ & $83(64.84)$ & $89(62.24)$ & 82 (57.75) & $71(60.68)$ & 85 (61.59) & $54(58.06)$ & $81(59.56)$ \\
\hline \multirow{2}{*}{$\begin{array}{l}\text { Perpetrated interpersonal } \\
\text { sexual violence }\end{array}$} & Victimized Male & $5(12.50)$ & $5(23.81)$ & $5(21.74)$ & $8(19.51)$ & $8(17.02)$ & $5(16.13)$ & $12(15.00)$ & 8 (19.05) \\
\hline & Non-Victimized Males & $12(8.82)$ & $10(7.81)$ & $9(6.29)$ & $8(5.67)$ & $6(5.13)$ & $10(7.25)$ & $4(4.35)$ & $8(5.93)$ \\
\hline \multirow{2}{*}{$\begin{array}{l}\text { Perpetrated intimate-partner } \\
\text { physical violence }\end{array}$} & Victimized Male & $11(28.21)$ & $9(42.86)$ & $8(34.78)$ & $8(19.51)$ & $7(15.22)$ & $7(23.33)$ & $11(13.92)$ & $6(14.29)$ \\
\hline & Non-Victimized Males & $10(7.46)$ & $9(7.09)$ & $9(6.38)$ & $12(8.76)$ & $11(9.57)$ & $11(8.03)$ & $8(8.79)$ & $14(10.53)$ \\
\hline \multirow{2}{*}{$\begin{array}{l}\text { Perpetrated intimate-partner } \\
\text { emotional violence }\end{array}$} & Victimized Male & $29(72.50)$ & $17(80.95)$ & $19(82.91)$ & 33 (80.49) & 38 (80.85) & $25(80.65)$ & $60(75.95)$ & $29(69.05)$ \\
\hline & Non-Victimized Males & $81(60.90)$ & $76(60.32)$ & $85(60.28)$ & $80(58.39)$ & $64(56.14)$ & $81(59.56)$ & $48(52.74)$ & $83(62.41)$ \\
\hline \multirow{2}{*}{$\begin{array}{l}\text { Perpetrated intimate-partner } \\
\text { sexual violence }\end{array}$} & Victimized Male & $12(29.27)$ & $6(28.57)$ & $7(30.43)$ & $11(26.83)$ & $9(19.15)$ & $8(25.81)$ & $16(20.00)$ & $11(26.19)$ \\
\hline & Non-Victimized Males & 15 (11.28) & $16(12.70)$ & 17 (12.06) & $15(10.87)$ & $13(11.40)$ & $17(12.50)$ & $10(10.99)$ & $16(11.94)$ \\
\hline
\end{tabular}

§Fisher's exact test was used where cell count was $\leq 10$; Values in bold indicate significant differences between male and female participants - $\mathrm{p}$-value $\leq 0.05$ 
Table 3.6: Unadjusted Odds Ratios for Relationship between Sexual Victimization and Aggressive Behaviors among Male Participants ${ }^{\S}$

\begin{tabular}{|c|c|c|c|c|c|c|c|c|c|}
\hline \multirow[t]{2}{*}{ Variables } & & \multicolumn{8}{|c|}{ OR $(95 \% C I) \ddagger$} \\
\hline & & $\begin{array}{l}\text { Sexual } \\
\text { Contact }\end{array}$ & Coercion & $\begin{array}{l}\text { Attempted } \\
\text { Rape }\end{array}$ & $\begin{array}{l}\text { Sexual Assault } \\
\text { (SA) with an } \\
\text { object }\end{array}$ & $\begin{array}{l}\text { Completed } \\
\text { Rape }\end{array}$ & $\begin{array}{l}\text { Drug or Alcohol } \\
\text { Given by } \\
\text { Perpetrator }\end{array}$ & $\begin{array}{c}\text { Drug or } \\
\text { Alcohol Use by } \\
\text { yourself }\end{array}$ & $\begin{array}{l}\text { External } \\
\text { Pressure }\end{array}$ \\
\hline \multirow[t]{2}{*}{$\begin{array}{l}\text { Perpetrated interpersonal } \\
\text { physical violence }\end{array}$} & Victimized Male & $\begin{array}{c}1.33 \\
(0.65,2.69)\end{array}$ & $\begin{array}{c}2.27 \\
(0.83,6.23)\end{array}$ & $\begin{array}{c}2.13 \\
(0.82,5.49)\end{array}$ & $\begin{array}{c}2.55 \\
(1.22,5.32)\end{array}$ & $\begin{array}{c}2.56 \\
(1.24,5.28)\end{array}$ & $\begin{array}{c}2.44 \\
(1.05,5.68)\end{array}$ & $\begin{array}{c}1.72 \\
(0.94,3.16)\end{array}$ & $\begin{array}{c}2.51 \\
(1.20,5.23)\end{array}$ \\
\hline & Non-Victimized Males & 1.00 & 1.00 & 1.00 & 1.00 & 1.00 & 1.00 & 1.00 & 1.00 \\
\hline \multirow[t]{2}{*}{$\begin{array}{l}\text { Perpetrated interpersonal } \\
\text { emotional violence }\end{array}$} & Victimized Male & $\begin{array}{c}1.63 \\
(0.75,3.54)\end{array}$ & $\begin{array}{c}3.25 \\
(0.90,11.63)\end{array}$ & $\begin{array}{c}2.18 \\
(0.76,6.22)\end{array}$ & $\begin{array}{c}2.60 \\
(1.15,5.85)\end{array}$ & $\begin{array}{c}1.89 \\
(0.89,4.01)\end{array}$ & $\begin{array}{c}3.24 \\
(1.17,8.96)\end{array}$ & $\begin{array}{c}1.58 \\
(0.84,2.97)\end{array}$ & $\begin{array}{c}2.17 \\
(0.98,4.78)\end{array}$ \\
\hline & Non-Victimized Males & 1.00 & 1.00 & 1.00 & 1.00 & 1.00 & 1.00 & 1.00 & 1.00 \\
\hline \multirow[t]{2}{*}{$\begin{array}{l}\text { Perpetrated interpersonal } \\
\text { sexual violence }\end{array}$} & Victimized Male & $\begin{array}{c}1.47 \\
(0.48,4.47)\end{array}$ & $\begin{array}{c}3.68 \\
(1.11,12.16)\end{array}$ & $\begin{array}{c}4.13 \\
(1.24,13.71)\end{array}$ & $\begin{array}{c}4.03 \\
(1.40,11.53)\end{array}$ & $\begin{array}{c}3.79 \\
(1.23,11.62)\end{array}$ & $\begin{array}{c}2.46 \\
(0.77,7.80)\end{array}$ & $\begin{array}{c}3.88 \\
(1.19,12.57)\end{array}$ & $\begin{array}{c}3.73 \\
(1.30,10.68)\end{array}$ \\
\hline & Non-Victimized Males & 1.00 & 1.00 & 1.00 & 1.00 & 1.00 & 1.00 & 1.00 & 1.00 \\
\hline \multirow[t]{2}{*}{$\begin{array}{l}\text { Perpetrated intimate- } \\
\text { partner physical violence }\end{array}$} & Victimized Male & $\begin{array}{c}4.87 \\
(1.88,12.59)\end{array}$ & $\begin{array}{c}9.83 \\
(3.27,29.49)\end{array}$ & $\begin{array}{c}7.82 \\
(2.62,23.30)\end{array}$ & $\begin{array}{c}2.52 \\
(0.95,6.68)\end{array}$ & $\begin{array}{c}1.67 \\
(0.61,4.69)\end{array}$ & $\begin{array}{c}3.48 \\
(1.22,9.92)\end{array}$ & $\begin{array}{c}1.67 \\
(0.63,4.40)\end{array}$ & $\begin{array}{c}1.41 \\
(0.50,3.95)\end{array}$ \\
\hline & Non-Victimized Males & 1.00 & 1.00 & 1.00 & 1.00 & 1.00 & 1.00 & 1.00 & 1.00 \\
\hline \multirow[t]{2}{*}{$\begin{array}{l}\text { Perpetrated intimate- } \\
\text { partner emotional violence }\end{array}$} & Victimized Male & $\begin{array}{c}1.69 \\
(0.77,3.67)\end{array}$ & $\begin{array}{c}2.79 \\
(0.88,8.79)\end{array}$ & $\begin{array}{c}3.12 \\
(1.01,9.68)\end{array}$ & $\begin{array}{c}2.93 \\
(1.26,6.83)\end{array}$ & $\begin{array}{c}3.29 \\
(1.46,7.45)\end{array}$ & $\begin{array}{c}2.82 \\
(1.08,7.34)\end{array}$ & $\begin{array}{c}2.82 \\
(1.46,5.47)\end{array}$ & $\begin{array}{c}1.34 \\
(0.64,2.82)\end{array}$ \\
\hline & Non-Victimized Males & 1.00 & 1.00 & 1.00 & 1.00 & 1.00 & 1.00 & 1.00 & 1.00 \\
\hline \multirow[t]{2}{*}{$\begin{array}{l}\text { Perpetrated intimate- } \\
\text { partner sexual violence }\end{array}$} & Victimized Male & $\begin{array}{c}3.25 \\
(1.37,7.70)\end{array}$ & $\begin{array}{c}2.75 \\
(0.93,8.11)\end{array}$ & $\begin{array}{c}3.19 \\
(1.14,8.87)\end{array}$ & $\begin{array}{c}3.00 \\
(1.25,7.20)\end{array}$ & $\begin{array}{c}1.84 \\
(0.72,4.65)\end{array}$ & $\begin{array}{c}2.43 \\
(0.94,6.30)\end{array}$ & $\begin{array}{c}2.02 \\
(0.86,4.76)\end{array}$ & $\begin{array}{c}2.61 \\
(1.10,6.20)\end{array}$ \\
\hline & Non-Victimized Males & 1.00 & 1.00 & 1.00 & 1.00 & 1.00 & 1.00 & 1.00 & 1.00 \\
\hline
\end{tabular}

$\S$ Values in bold indicate significant differences between male and female participants - $\mathrm{p}$-value $\leq 0.05$;

$\ddagger$ OR $=$ Odds Ratio; $\mathrm{CI}=$ Confidence Interval 
Table 3.7: Adjusted Odds Ratios for Relationship between Sexual Victimization and Aggressive Behaviors among Male Participants

\begin{tabular}{|c|c|c|c|c|c|c|c|c|c|}
\hline \multirow[t]{2}{*}{ Variables } & & \multicolumn{8}{|c|}{ Adjusted $\mathrm{OR}^{\S}(95 \% \mathrm{CI})$} \\
\hline & & Sexual Contact & Coercion & $\begin{array}{l}\text { Attempted } \\
\text { Rape }\end{array}$ & $\begin{array}{l}\text { Sexual Assault } \\
\text { (SA) with an } \\
\text { object }\end{array}$ & $\begin{array}{l}\text { Completed } \\
\text { Rape }\end{array}$ & $\begin{array}{c}\text { Drug or } \\
\text { Alcohol Given } \\
\text { by Perpetrator }\end{array}$ & $\begin{array}{l}\text { Drug or } \\
\text { Alcohol Use } \\
\text { by yourself }\end{array}$ & $\begin{array}{l}\text { External } \\
\text { Pressure }\end{array}$ \\
\hline \multirow[t]{2}{*}{$\begin{array}{l}\text { Perpetrated interpersonal } \\
\text { physical violence }\end{array}$} & Victimized Male & $\begin{array}{c}1.44 \\
(0.69,2.98)\end{array}$ & $\begin{array}{c}2.31 \\
(0.81,6.52)\end{array}$ & $\begin{array}{c}2.46 \\
(0.91,6.69)\end{array}$ & $\begin{array}{c}2.56 \\
(1.20,5.46)\end{array}$ & $\begin{array}{c}2.70 \\
(1.27,5.73)\end{array}$ & $\begin{array}{c}2.60 \\
(1.07,6.34)\end{array}$ & $\begin{array}{c}1.87 \\
(0.99,3.53)\end{array}$ & $\begin{array}{c}2.54 \\
(1.19,5.41)\end{array}$ \\
\hline & Non-Victimized Males & 1.00 & 1.00 & 1.00 & 1.00 & 1.00 & 1.00 & 1.00 & 1.00 \\
\hline \multirow[t]{2}{*}{$\begin{array}{l}\text { Perpetrated interpersonal } \\
\text { emotional violence }\end{array}$} & Victimized Male & $\begin{array}{c}1.75 \\
(0.78,3.91)\end{array}$ & $\begin{array}{c}3.26 \\
(0.88,12.04)\end{array}$ & $\begin{array}{c}2.43 \\
(0.81,7.26)\end{array}$ & $\begin{array}{c}2.57 \\
(1.12,5.89)\end{array}$ & $\begin{array}{c}1.86 \\
(0.86,4.06)\end{array}$ & $\begin{array}{c}3.35 \\
(1.15,9.72)\end{array}$ & $\begin{array}{c}1.65 \\
(0.86,3.16)\end{array}$ & $\begin{array}{c}2.15 \\
(0.96,4.81)\end{array}$ \\
\hline & Non-Victimized Males & 1.00 & 1.00 & 1.00 & 1.00 & 1.00 & 1.00 & 1.00 & 1.00 \\
\hline \multirow[t]{2}{*}{$\begin{array}{l}\text { Perpetrated interpersonal } \\
\text { sexual violence }\end{array}$} & Victimized Male & $\begin{array}{c}1.47 \\
(0.46,4.60)\end{array}$ & $\begin{array}{c}4.06 \\
(1.18,13.98)\end{array}$ & $\begin{array}{c}4.48 \\
(1.29,15.54)\end{array}$ & $\begin{array}{c}4.45 \\
(1.48,13.40)\end{array}$ & $\begin{array}{c}4.81 \\
(1.49,15.52)\end{array}$ & $\begin{array}{c}2.33 \\
(0.70,7.69)\end{array}$ & $\begin{array}{c}4.54 \\
(1.34,15.40)\end{array}$ & $\begin{array}{c}4.17 \\
(1.38,12.63)\end{array}$ \\
\hline & Non-Victimized Males & 1.00 & 1.00 & 1.00 & 1.00 & 1.00 & 1.00 & 1.00 & 1.00 \\
\hline \multirow[t]{2}{*}{$\begin{array}{l}\text { Perpetrated intimate- } \\
\text { partner physical violence }\end{array}$} & Victimized Male & $\begin{array}{c}5.76 \\
(2.16,15.35)\end{array}$ & $\begin{array}{c}12.15 \\
(3.74,39.43)\end{array}$ & $\begin{array}{c}10.09 \\
(3.18,31.99)\end{array}$ & $\begin{array}{c}3.07 \\
(1.10,8.51)\end{array}$ & $\begin{array}{c}2.19 \\
(0.76,6.32)\end{array}$ & $\begin{array}{c}4.08 \\
(1.34,12.37)\end{array}$ & $\begin{array}{c}2.01 \\
(0.74,5.45)\end{array}$ & $\begin{array}{c}1.57 \\
(0.53,4.58)\end{array}$ \\
\hline & Non-Victimized Males & 1.00 & 1.00 & 1.00 & 1.00 & 1.00 & 1.00 & 1.00 & 1.00 \\
\hline \multirow[t]{2}{*}{$\begin{array}{l}\text { Perpetrated intimate- } \\
\text { partner emotional violence }\end{array}$} & Victimized Male & $\begin{array}{c}1.80 \\
(0.80,4.04)\end{array}$ & $\begin{array}{c}2.70 \\
(0.84,8.61)\end{array}$ & $\begin{array}{c}3.52 \\
(1.07,11.53)\end{array}$ & $\begin{array}{c}2.97 \\
(1.25,7.07)\end{array}$ & $\begin{array}{c}3.27 \\
(1.41,7.55)\end{array}$ & $\begin{array}{c}3.01 \\
(1.11,8.17)\end{array}$ & $\begin{array}{c}3.01 \\
(1.50,6.04)\end{array}$ & $\begin{array}{c}1.26 \\
(0.58,2.70)\end{array}$ \\
\hline & Non-Victimized Males & 1.00 & 1.00 & 1.00 & 1.00 & 1.00 & 1.00 & 1.00 & 1.00 \\
\hline \multirow[t]{2}{*}{$\begin{array}{l}\text { Perpetrated intimate- } \\
\text { partner sexual violence }\end{array}$} & Victimized Male & $\begin{array}{c}3.71 \\
(1.49,9.23)\end{array}$ & $\begin{array}{c}3.47 \\
(1.09,11.04)\end{array}$ & $\begin{array}{c}3.55 \\
(1.20,10.45)\end{array}$ & $\begin{array}{c}4.15 \\
(1.61,10.68)\end{array}$ & $\begin{array}{c}2.58 \\
(0.95,6.96)\end{array}$ & $\begin{array}{c}2.91 \\
(1.04,8.13)\end{array}$ & $\begin{array}{c}2.83 \\
(1.17,7.19)\end{array}$ & $\begin{array}{c}3.59 \\
(1.39,9.26)\end{array}$ \\
\hline & Non-Victimized Males & 1.00 & 1.00 & 1.00 & 1.00 & 1.00 & 1.00 & 1.00 & 1.00 \\
\hline
\end{tabular}


Table 3.8: Prevalence of Suicidal Behaviors and Deliberate Self-Harm (DSH) among Victimized Males and Females ${ }^{\S}$

\begin{tabular}{|c|c|c|c|c|c|c|c|c|c|}
\hline \multirow[t]{2}{*}{ Variables } & & \multicolumn{8}{|c|}{$\begin{array}{c}\text { Types of Victimization } \\
n(\text { Percentage })\end{array}$} \\
\hline & & $\begin{array}{l}\text { Sexual } \\
\text { Contact }\end{array}$ & Coercion & $\begin{array}{l}\text { Attempted } \\
\text { Rape }\end{array}$ & $\begin{array}{l}\text { Sexual Assault } \\
\text { (SA) with an } \\
\text { object }\end{array}$ & $\begin{array}{l}\text { Completed } \\
\text { Rape }\end{array}$ & $\begin{array}{c}\text { Drug or Alcohol } \\
\text { Given by } \\
\text { Perpetrator }\end{array}$ & $\begin{array}{l}\text { Drug or Alcohol } \\
\text { use by yourself }\end{array}$ & $\begin{array}{l}\text { External } \\
\text { Pressure }\end{array}$ \\
\hline \multirow[t]{2}{*}{ Suicidal Behaviors } & Victimized Male & $12(28.57)$ & $11(52.38)$ & $10(43.48)$ & $10(24.39)$ & $13(27.66)$ & $11(35.48)$ & $16(20.00)$ & $14(33.33)$ \\
\hline & Victimized Female & $84(34.85)$ & $66(40.74)$ & $67(38.29)$ & $55(34.16)$ & $70(36.46)$ & $43(36.44)$ & $80(30.89)$ & $36(34.95)$ \\
\hline \multirow[t]{2}{*}{ DSH } & Victimized Male & $23(57.50)$ & 17 (80.95) & $16(72.73)$ & $25(60.98)$ & $26(55.32)$ & $21(67.74)$ & $44(55.00)$ & $28(70.00)$ \\
\hline & Victimized Female & $133(56.12)$ & $94(58.75)$ & $95(55.23)$ & $93(57.76)$ & $110(57.29)$ & $70(59.83)$ & $130(50.39)$ & $55(53.92)$ \\
\hline
\end{tabular}

§Values in bold indicate significant differences between male and female participants - $\mathrm{p}$-value $\leq 0.05$

Table 3.9: Unadjusted Odds Ratios for Relationship between Sexual Victimization and Suicidal Behaviors and Deliberate Self-Harm (DSH)

\begin{tabular}{|c|c|c|c|c|c|c|c|c|c|}
\hline \multirow[t]{2}{*}{ Variables } & & \multicolumn{8}{|c|}{ OR $(95 \% C I) \ddagger$} \\
\hline & & $\begin{array}{l}\text { Sexual } \\
\text { Contact }\end{array}$ & Coercion & $\begin{array}{c}\text { Attempted } \\
\text { Rape }\end{array}$ & $\begin{array}{l}\text { Sexual Assault } \\
\text { (SA) with an } \\
\text { object }\end{array}$ & $\begin{array}{c}\text { Completed } \\
\text { Rape }\end{array}$ & $\begin{array}{c}\text { Drug or Alcohol } \\
\text { Given by } \\
\text { Perpetrator }\end{array}$ & $\begin{array}{l}\text { Drug or Alcohol } \\
\text { use by yourself }\end{array}$ & $\begin{array}{l}\text { External } \\
\text { Pressure }\end{array}$ \\
\hline Suicidal Behaviors & $\begin{array}{c}\text { Victimized Male } \\
\text { Victimized Female }\end{array}$ & $\begin{array}{c}1.33 \\
(0.65,2.74) \\
1.00\end{array}$ & $\begin{array}{c}0.62 \\
(0.25,1.55) \\
1.00\end{array}$ & $\begin{array}{c}0.80 \\
(0.33,1.94) \\
1.00\end{array}$ & $\begin{array}{c}1.60 \\
(0.73,3.52) \\
1.00\end{array}$ & $\begin{array}{c}1.50 \\
(0.74,3.03) \\
1.00\end{array}$ & $\begin{array}{c}1.04 \\
(0.45,2.38) \\
1.00\end{array}$ & $\begin{array}{c}1.78 \\
(0.97,3.28) \\
1.00\end{array}$ & $\begin{array}{c}1.07 \\
(0.50,2.29) \\
1.00\end{array}$ \\
\hline DSH & $\begin{array}{c}\text { Victimized Male } \\
\text { Victimized Female }\end{array}$ & $\begin{array}{c}0.94 \\
(0.48,1.86) \\
1.00\end{array}$ & $\begin{array}{c}0.33 \\
(0.10,1.04) \\
1.00\end{array}$ & $\begin{array}{c}0.46 \\
(0.17,1.23) \\
1.00\end{array}$ & $\begin{array}{c}0.87 \\
(0.43,1.76) \\
1.00\end{array}$ & $\begin{array}{c}1.08 \\
(0.57,2.05) \\
1.00\end{array}$ & $\begin{array}{c}0.70 \\
(0.30,1.64) \\
1.00\end{array}$ & $\begin{array}{c}0.83 \\
(0.50,1.37) \\
1.00\end{array}$ & $\begin{array}{c}0.50 \\
(0.23,1.09) \\
1.00\end{array}$ \\
\hline
\end{tabular}

$\ddagger$ OR $=$ Odds Ratio; $\mathrm{CI}=$ Confidence Interval

Table 3.10: Adjusted Odds Ratios for Relationship between Sexual Victimization and Suicidal Behaviors and Deliberate Self-Harm (DSH)

\begin{tabular}{|c|c|c|c|c|c|c|c|c|c|}
\hline \multirow[t]{2}{*}{ Variables } & & \multicolumn{8}{|c|}{ Adjusted OR $(95 \%$ CI $) \ddagger$} \\
\hline & & $\begin{array}{l}\text { Sexual } \\
\text { Contact }\end{array}$ & Coercion & $\begin{array}{c}\text { Attempted } \\
\text { Rape }\end{array}$ & $\begin{array}{c}\text { Sexual Assault } \\
\text { (SA) with an } \\
\text { object }\end{array}$ & $\begin{array}{c}\text { Completed } \\
\text { Rape }\end{array}$ & $\begin{array}{c}\text { Drug or Alcohol } \\
\text { Given by } \\
\text { Perpetrator }\end{array}$ & $\begin{array}{c}\text { Drug or Alcohol } \\
\text { use by yourself }\end{array}$ & $\begin{array}{l}\text { External } \\
\text { Pressure }\end{array}$ \\
\hline Suicidal Behaviors & $\begin{array}{c}\text { Victimized Male } \\
\text { Victimized Female }\end{array}$ & $\begin{array}{c}1.38 \\
(0.65,2.92) \\
1.00\end{array}$ & $\begin{array}{c}0.57 \\
(0.22,1.47) \\
1.00\end{array}$ & $\begin{array}{c}0.76 \\
(0.30,1.90) \\
1.00\end{array}$ & $\begin{array}{c}1.38 \\
(0.60,3.15) \\
1.00\end{array}$ & $\begin{array}{c}1.40 \\
(0.67,2.91) \\
1.00\end{array}$ & $\begin{array}{c}1.14 \\
(0.47,2.73) \\
1.00\end{array}$ & $\begin{array}{c}1.76 \\
(0.93,3.31) \\
1.00\end{array}$ & $\begin{array}{c}1.04 \\
(0.46,2.34 \\
1.00\end{array}$ \\
\hline DSH & $\begin{array}{c}\text { Victimized Male } \\
\text { Victimized Female }\end{array}$ & $\begin{array}{c}0.88 \\
(0.44,1.77) \\
1.00\end{array}$ & $\begin{array}{c}0.32 \\
(0.10,1.04) \\
1.00\end{array}$ & $\begin{array}{c}0.46 \\
(0.17,1.25) \\
1.00\end{array}$ & $\begin{array}{c}0.68 \\
(0.32,1.45) \\
1.00\end{array}$ & $\begin{array}{c}1.03 \\
(0.53,1.99) \\
1.00\end{array}$ & $\begin{array}{c}0.65 \\
(0.26,1.60) \\
1.00\end{array}$ & $\begin{array}{c}0.80 \\
(0.47,1.34) \\
1.00\end{array}$ & $\begin{array}{c}0.47 \\
(0.20,1.08 \\
1.00\end{array}$ \\
\hline
\end{tabular}

‡ Odds Ratio (OR) adjusted for age, year in school, race, and current relationship status; CI = Confidence Interval 
Chapter 4 


\section{Chapter 4}

\subsection{Introduction}

A recent review of literature suggested that terms like "sexual assault" or "sexual violence" have been used by researchers to describe a broad range of sexual acts including forced touching or kissing; verbally coerced intercourse; and physically forced vaginal, oral or anal penetration. ${ }^{3}$ The Centers for Disease Control and Prevention's (CDC) National Center for Injury Prevention and Control (NCIPC) developed the Sexual Violence Surveillance: Uniform Definitions and Recommended Data Elements (Version 1.0), which defined sexual violence as any sexual act that is forced against someone's will. Consistent with past use, the CDC definition of sexual violence can include any physical, verbal, or psychological act. ${ }^{1}$

Sexual victimization is a significant public health problem affecting both men and women. According to estimates obtained from a sample of US emergency departments, nearly 75,000 people (Age adjusted rate: 25.32/100,000) were treated for injuries resulting from a sexual assault in $2007 .{ }^{153}$ The rate of injuries among the college-age population was markedly higher. In that same year, individuals between the ages of 17-24 years accounted for almost one-third $(n=20.965$; rate: $54.89 / 100,000)$ of all injuries associated with sexual assault. While a majority of those between the ages of 17-24 years treated for injuries associated with sexual assault were female $(n=19,695$; rate: $106.31 / 100,000)$, males were also treated $(n=1,271$; rate:

$6.46 / 100,000){ }^{153}$

Existing studies have documented the prevalence of different types of sexual assault among college populations. Findings from a national survey of 3,000 college-attending women reported that $54 \%$ of women in this sample had experienced some form of sexual violence victimization. ${ }^{154}$ According to estimates obtained from the National College Women Sexual 
Victimization survey (1997), rates of attempted and completed forced sexual intercourse among college women were 11 and 16.6 per 1,000 female students, respectively. ${ }^{155}$ A more recent study of college women reported that $38 \%$ of participants had experienced one or more episodes of sexual victimization. ${ }^{11}$ Rape is generally believed to be the most common type of sexual assault victimization experienced by women. However, studies have suggested that both men and women may experience other forms of sexual violence. ${ }^{3,129,156,157}$ Although smaller in number, studies examining the prevalence of sexual victimization among college-attending males have suggested that $5 \%-34 \%$ of male university students experienced some form sexual victimization. $^{15,23-25}$ Another study of the prevalence of male sexual assault in the student population suggested that $14 \%$ of males (compared with $24 \%$ females) had experienced an unwanted sexual experience at least once in their lifetime. ${ }^{22}$ A study of college-aged men reported that $6 \%$ of participants had experienced rape perpetrated by an acquaintance and $17 \%$ had experienced an unwanted sexual contact. ${ }^{25}$

It is often difficult to compare the findings of different studies because differences in definitions of sexual victimization used or type of measure used to identify sexual victimization. A majority of existing studies have focused on the most severe type of victimization (attempted or completed forced sexual intercourse). However, existing literature has suggested that a large range of unwanted sexual experiences may be associated with poor mental and physical health. ${ }^{40 \text {, }}$ 71,92, 147 For example, both male and female victims of sexual violence are more likely to report depression, suicide ideation and substance abuse than those who have not been victimized. ${ }^{20,52 \text {, }}$ 58, 92, 126, 158 Researchers has also examined the relationships between severity of victimization and negative health outcomes. Findings from qualitative interviews have indicated that negative consequences (e.g. depression, low sexual esteem) were reported by men who experienced 
unwanted sexual contact without penetration. ${ }^{116}$ A study by Choudhary et al. (2009) indicated that adverse health outcomes experienced by males varied by the type (or category) of reported sexual violence victimization. ${ }^{159}$

Sexual victimization is often perceived as an issue that is more salient to women. However, studies of sexual victimization often include males and females in the same sample design. Before results from existing studies can be compared or used to inform current knowledge of the causes and correlates of sexual violence, it is important to understand definitional differences that may be associated with victimization or gender. Because men and women may differ in their definitions of sexual violence, and the strong likelihood that victimization may affect understanding of sexual violence, it is important to explore how definitions of sexual victimization differ between men and women. One possibility is that the definitions of sexual violence can be rooted in gender and modified by victimization. A conceptual model detailing the proposed model of the relationship between, gender, victimization, and definitions of unwanted sexual experience is shown in Figure 4.1. To address gaps in current understanding of victimization- and gender-based differences in definitions of sexual violence, the current study collected data on definitions of sexual victimization from a sample of college-attending adolescents and young adults. The purpose of this study was to identify differences and similarities in the definition of unwanted sexual experience between males and females and whether definitions differed according to history of victimization. 


\subsection{Methods}

\subsubsection{Design}

Data was collected from a convenience sample of undergraduate psychology students using a self-administered questionnaire containing standardized items and previously developed and validated scales to measure sexual violence. The questionnaire was designed to collect data on the relationships between unwanted sexual experiences, physical and mental health outcomes, and risk behaviors. We used the Sexual Experience Survey (SES) to assess sexual violence victimization. ${ }^{160}$ The SES is the most frequently used of all similar measures assessing unwanted sexual experience. This instrument was designed for university students and previous studies have indicated that the instrument has good internal consistency (Cronbach's $\alpha=0.74$ ) and good test-retest reliability $(\mathrm{r}=0.93) .{ }^{160,161}$ Demographic questions, adapted from American College Health Association's National College Health Assessment Survey, included measures of gender, age, class rank (year of study), race, and ethnic background. ${ }^{133}$ An open-ended question asking participants to define an "unwanted sexual experience" was used to identify differences in definitions of unwanted sexual experiences. Responses to this question were used to examine the similarities and differences in the definition of unwanted sexual experience using a grounded theory approach to the identification of emerging themes. ${ }^{162,163}$

\subsubsection{Participants}

Male and female students were recruited from undergraduate psychology courses at a large public university in the Mid-Atlantic region of the United States. The survey was administered from December 2008 to March 2009. Students in four psychology undergraduate classes (Introduction to Psychology, Introduction to Human Development, Introduction to Social Psychology, and Introduction to Abnormal Psychology) learned about the research opportunity 
during a brief explanation of the study during normal class hours. An informational flyer about the study was also displayed on the Psychology Department's general bulletin board.

Participants completed the self-administered questionnaire via West Virginia University’s online

research system. ${ }^{130}$ This system provides web-based human subject pool management capability for university-affiliated researchers. Students participating in this study received extra credit applied to their course grade. While researchers are free to provide additional credit for participation, the amount of extra credit awarded for participation in research is standardized across studies at two points. Students consented to participation via the online system, and were given access to information about their rights as participants in the study. This study was approved by the university's Institutional Review Board (IRB).

To establish history of sexual violence victimization, male and female participants were asked a series of questions about unwanted sexual activity using the SES survey. Responses to questions about different categories of sexual violence were then used to define a history of victimization. Those who answered "yes" to questions asking if they ever experienced attempted sexual contact and/or unwanted participation in sex acts such as anal or oral intercourse or penetration by an object other than the penis and/or completed unwanted sexual intercourse by a male or female perpetrator were considered to have been victimized and those who answered "no" to all questions about unwanted sexual experiences formed the non-victimized reference group.

\subsubsection{Data Analysis}

Responses to the open-ended question asking each participant to define his/her understanding of an "unwanted sexual experience" were examined using a grounded theory method. ${ }^{162,163}$ Each response was then read to identify emerging themes by identifying words and phrases from text that appeared to capture key thoughts or concepts. All transcripts were first read by the author 
and independent collaborator. Responses were first open coded, which is an inductive process in which the researcher dissects information on the basis of emerging or repetitive features in the text. To establish reliability and for the purpose of comparison, a list of main themes and representative quotations was prepared by each researcher. Instances of disagreement were resolved through a process of discussion and negotiation. Once the emergent categories were saturated (no new themes were observed), these categories were organized into meaningful core groupings. Once the initial list of groupings was finalized, we engaged in axial coding, which involved refining the list by deleting or merging some categories. This process was followed by making connections between categories (Figure 4.2). Our analysis of the data yielded three core categories (type of unwanted sexual activities, why it was unwanted, and emotions expressed towards unwanted sexual experience) which were further divided into multiple groups (Figure 4.2). Finally, content-analysis summary tables were used as an organizational tool to construct a profile of group membership. ${ }^{164}$ Organization of the categories was based on the demographic and victimization characteristics of the participants using the qualitative data analysis software QSR Nvivo (Ver 8.0).

\subsection{Results}

A total of 575 participants answered the open-ended question asking about definitions of unwanted sexual experiences. Among all participants, there were 76 victimized males, 296 victimized females, 68 non-victimized males and 135 non-victimized females. Detailed demographic information for each group is presented in Table 4.1. Overall, demographic characteristics were similar for all four groups. The majority of participants in each group were between the ages of 18-19 years, white, within the first 2 years of their college education, single, and identified themselves as heterosexual (Table 4.1). 
When examining definitions of unwanted sexual experiences provided by all participants, three main categories emerged: type of unwanted sexual experience, why it was unwanted, and emotions or feelings expressed towards the unwanted sexual experiences. Most of the participants described their definitions as a mixture of these three categories. For example, one of the participants described an unwanted sexual experience as: "Molestation, rape, unwanted touching/grabbing, drunk sexual experience that (after sobering up) you wished you hadn't done.” This example provides evidence of two main categories: types of unwanted sexual experience - "Molestation, rape, unwanted touching/grabbing" and why unwanted - "drunk sexual experience that (after sobering up) you wished you hadn't done". Another respondent described an unwanted sexual experience as "a sexual experience in which you do not want to have sex and felt uncomfortable, sad, angry, or used before, during, or after sexual intercourse or a sexual act". This example shows the third major theme: emotions or feeling expressed towards the unwanted sexual experience such as "uncomfortable, sad, angry, or used." Similar themes were observed in other responses and were coded accordingly.

\subsubsection{Types of Unwanted Sexual Experience}

A comparison of types of unwanted sexual experienced provided by victimized and nonvictimized participants are shown in Table 4.2. Both males and females, regardless of victimization, described a wide range of unwanted sexual experiences. The unwanted sexual experience most frequently described was rape. This one word description was frequently provided by both male and female participants. Regardless of gender or victimization, a majority of the participants also described unwanted sexual experience as "any sexual activity or experience". This category was used to describe all responses where words like "any sexual act" or "any sexual experience" or "anything sexual". Another category that was common among 
male and female participants was unwanted "touching" in a sexual manner. This category included responses where the participant described an unwanted sexual experience as "touching, kissing, grabbing" (victimized male), "any form of touching” (non-victimized male), "touching or sexual contact" (victimized female), or "any unwanted touching in sexual manner" (nonvictimized female).

When comparing the descriptions of "types of unwanted sexual experience," it was noted that victimized males, victimized females, and non-victimized females used phrases such as "performing a sex act" or "any sexual act that was given or received." The same was not true for non-victimized males.

Victimized male: "When one person performs sexual actions when they do not want to..."

Victimized female: "An unwanted sexual experience is any type of sex (oral, vaginal, anal) that you gave or received that you did not want."

Non-victimized female: "Someone forcing you to do anything sexual with them when you don't want to"

Overall, female participants provided a wider range of unwanted sexual experiences than males. Victimized and non-victimized females had some common themes in their descriptions of type of unwanted sexual experiences. Female participants described unwanted sexual activity as sexual aggression, sexual behavior, taking advantage, and verbal abuse. These themes were not observed among male participants.

Victimized female: "Any experience in which someone has been physically or verbally subjected to something that was uncomfortable relating to sex." 
Non-victimized female: "Being forced to have sex or someone taking advantage of you...."

The description of $\mathrm{n}$ unwanted sexual experience provided by women who had experienced sexual victimization showed additional themes describing the type of unwanted sexual activity. One victimized female described unwanted sexual activity as "any act of affection or infatuation...", "anything involved with your privates...", and "flirting."

Few of the themes that emerged in the descriptions provided by non-victimized females were not observed among other participants. One non-victimized female described a type of unwanted sexual activity as "revealing of a penis, vagina, or breasts...." Another non-victimized female participant described an unwanted sexual activity as "being looked at in a way I do not want"

\subsubsection{Why Unwanted}

In addition to describing the type of sexual act that was unwanted, male and female participants also included why the sexual experience was unwanted. Comparisons of this theme are provided in Table 4.3. There were many common elements shared by both males and females. A majority of all participants described an unwanted sexual experience as one that was unwanted because it had happened against their will or was not desired.

Victimized males: "'Sexual contact of any kind being physical, visual, or verbal that is not desired"; "An experience where one party engages in sex or sex related activities against that person's wishes"

Non-victimized male: "Against the will of another. Causes trauma and stress"; "Not wanting to have sex, but having sex anyway" 
Victimized female: "Having someone do something to you sexually against your will"; "Sex against your own will"

Non-victimized female: "Something that you did not want to engage in"; "Anything you are not willing to do"

Another theme that was observed among both males and females was coercion. This theme covered various types of coercive tactics used by the perpetrator such as pressure, begging, persuasion, excessive convincing, and persistence. Males who had experienced sexual victimization described an unwanted sexual experience where they "participated due to external pressure" or "sex you didn't want but were pressured into, don't have to be physically forceful, could use begging." Victimized females described coercion as "when someone pressured you some way" or "emotionally forced into sex." Similarly, non-victimized males indicated that a sexual experience is unwanted because the "other persuaded to do so" and "excessively convince to have sex or sexual act." Non-victimized females described an unwanted sexual experience as "pressured into doing [some] thing you don't want to do".

Many participants used the word "forced" when they were describing unwanted sexual experience (see Table 4.3). Another common theme that was seen in the descriptions provided by participants was any sexual experience that occurred "without consent".

Victimized males: "touching, kissing, or sex that is not consented"

Non-victimized male: "a sexual experience without your permission"

Victimized female: "a sexual experience performed by either partner without consent"

Non-victimized female: "sexual act that was conducted without my approval" 
Another theme that was common among male and female participants was an unwanted sexual experience in a relationship.

Victimized males: "Which at least one person does not wish to be involved in"; "One partner not wanting to have sexual intercourse"

Non-victimized males: "Anything sexual that is not wanted by one or both people"; "Either the male or female in the relationship not wanting to have a sexual experience occur"

Victimized females: "Anything that is not desired by either partner"; "Any type of intercourse where one partner does not want it"

Non-victimized females: "When someone doesn't want to have sex, but [their] partner forces them without their consent"; "If one of the partners does not [wish] to participate (usually a girl), to do something in a sexual manner (hugging, fondling, kissing, etc.)",

Victimized males and females (both victimized and non-victimized) described an unwanted sexual experience as one performed under the influence of alcohol or other substances and when a person was unable to make a clear judgment.

Victimized males: "Being too intoxicated to realize, having sex with a girl when [you're] drunk and thinking it was great, just to wake up in the morning to trailer trash"; "Something that I would [have] never done sober and that makes me feel disgusted when I think about it"

Victimized females: "Occurs under circumstances where one person is severely intoxicated"; "Sex when you were under the influence of drugs or alcohol"

Non-victimized female: "Taking advantage of you while you are under the influence of drugs or alcohol" 
Male participants also described an unwanted sexual experience as something that was not enjoyed. Additionally, victimized males described unwanted sexual experience as something that they were tricked into or something that they did not want to happen even when they verbally allowed it. One victimized male described an unwanted sexual experience as a “mistake." Victimized female participants described an unwanted sexual experience as something that they were "encouraged into" or something they "feel obligated to do" even if they did not want to. One female victim described an unwanted sexual experience as one where the person is "restrained." Another victimized female described an unwanted sexual experience where "a threat or injury causing someone to give into sex acts or intercourse." Whereas nonvictimized females described an unwanted sexual experience as "unwelcomed" "inappropriate" and "something you are not ready for."

\subsubsection{Emotions or Feelings Expressed towards Unwanted Sexual Experience}

Certain words emerged that were used to describe the feelings or emotions related to an unwanted sexual experience (see Table 4.4). Victimized males used words like "disgusted" or "sad" or "uncomfortable." In addition to these, victimized females used words like "angry," “difficult," "painful," "scary," and "harmful." Non-victimized males described unwanted sexual experiences as "uncomfortable," whereas non-victimized females used words like "horrible," "hurt," and "scarring." Both victimized and non-victimized participants also indicated that a sexual experience is unwanted if you regret it during or after the fact.

Victimized males: "Something that involves sex with another person that you regret or do not wish to partake in at the time of the occurrence"

Non-victimized male: "A sexual experience I regret" 
Victimized female: "Being with someone then feeling like it was a bad idea later"

Non-victimized female: "Something that at the time you thought you wanted, but in actually reality you regret after the experience"

\subsection{Discussion}

It has been estimated that sexual victimization affects a large number of college-attending men and women each year. Over the years, researchers have used a number of differing definitions of unwanted sexual experiences in order to study the prevalence and consequences of sexual violence victimization. The primary aim of this study was to explore differences in how collegeattending males and females differed in their definitions of an unwanted sexual experience. Results of this study highlight various similarities and identify some key differences in definitions of unwanted sexual experiences among males and females. As shown by the conceptual model (Figure 4.1), the differences in the definitions of unwanted sexual experience may be rooted in gender and similarities may be rooted in individual experience and the trauma associated with sexual victimization.

Our results showed that the definitions of unwanted sexual experience ranged from unwanted flirting to forced sexual intercourse. Female participants were more descriptive in their definitions and considered unwanted flirting as type of unwanted sexual experience when compared to male participants. Victimized males and females provided examples of unwanted sexual experiences that included greater detail and points of distinction when compared with non-victimized participants. As shown by the conceptual model (Figure 4.1), these differences may be rooted in individual experience and the trauma associated with sexual victimization. 
The most common description of unwanted sexual experience used by males and females (victimized and non-victimized) was "rape", which is usually considered as the most severe type of sexual violence. ${ }^{36}$ A majority of participants (24.78\%) used this one word description of an unwanted sexual experience suggesting that young males and females may lack awareness about other forms of sexual victimization. The wide range of other types of unwanted sexual experiences in the definitions provided by other males and females suggest that there is a need to examine the prevalence and correlates of different types of sexual victimization beyond forced sexual intercourse.

Women have been shown to be the primary victims of unwanted sexual experiences, especially when physical force is used by a perpetrator. Studies of commonly held perceptions related to sexual assault have reported that males are less likely to be considered victims or are more likely to be blamed for their victimization. ${ }^{32,165}$ Despite possible differences in perceptions of responsibility, our findings suggest that victimized males and females considered similar types of sexual experiences as unwanted and also provided similar descriptions of why they think that it was unwanted.

Studies that have used small clinical samples to examine the characteristics of male sexual assault have described a typical male victim as one who has sustained physical injuries during the assault, was threatened and had a weapon used against him. ${ }^{19,20,28}$ In contrast, studies that have surveyed males and females in the general population have suggested that a majority of male victims do not sustain physical injuries or have a weapon used against them during the assault. $^{15,166}$ Findings from college-based survey studies have also shown that, similar to women, men may also experience sexual coercion. ${ }^{167,168}$ Similar to these other studies, findings from this study suggest that male victims considered less physical tactics such as coercion and 
use of alcohol or unwanted participation in sexual acts with a partner as an unwanted sexual experience.

There is strong evidence linking personal alcohol use and sexual violence victimization., 3 , 85, 92, 128, 151,158 As shown by our results, both victimized males and females described an unwanted sexual experience where one is heavily intoxicated or consumed too much alcohol and were unaware of their actions. Both male and female victims' descriptions indicated that they would not have participated in a sexual act if they were not under the influence or alcohol. It is noteworthy that non-victimized males did not identify the role of alcohol in their definitions of unwanted sexual experience.

Another difference, which was observed among male and female participants' definitions of an unwanted sexual experience, was that only male participants indicated that a sexual experience that they did not enjoy was considered as unwanted. In their definition of an unwanted sexual experience, only victimized males and females indicated that not only receiving and but performing a sexual act can also be unwanted. In contrast, definitions of non-victimized males and females indicated that unwanted sexual acts are more likely to be perceived as something that a person receives.

Dating or relationship violence has been shown to be prevalent among college-aged men and women. ${ }^{128,149}$ Existing studies have indicated that physical and sexual violence is perpetrated and experienced by both men and women in a relationship. ${ }^{149,155,169}$ Previous studies have also shown that perpetrators of sexual assault among both men and women are more likely to be someone known to the victim. ${ }^{4,5}$ These findings are supported by results from this study as both male and female participants commonly described the role of their partner in their description of 
an unwanted sexual experience (e.g. when one partner was not interested, participated in an unwanted sexual act because partner wanted to, partner convinced excessively).

We observed that even though victimized males and females use similar words like "sad" and "uncomfortable" when describing unwanted sexual experience, victimized females used terms like "anger", "painful”, "unhappy” and "violated” to express their feelings towards unwanted sexual experiences. Gender differences in the psychological response to sexual assault are not clear. Previous researchers have argued that similar to women, men who experience sexual assault may exhibit a similar emotional response (e.g. feeling of fear, anger, anxiety etc.). ${ }^{6,7,40,71}$ While other studies have suggested that victimized males do not display similar emotions as victimized females. ${ }^{6,68}$ This inconsistency highlights the need for additional research to enhance understanding of gender-based differences in response to sexual victimization. A clear understanding of these differences, if any, will allow for a more comprehensive understanding of the psychological consequences of sexual assault and identify opportunities for early intervention and treatment.

One possible explanation of the observed gender differences in male and female definitions of an unwanted sexual experience can be the traced to hypothesized development of men and women proposed by Gilligan (1982). ${ }^{170,171}$ Gilligan's Theory of Moral Development posits that genderoriented differences in socialization during early childhood and normative reproductions of social and economic roles result in the development of gendered views of what is right and wrong or what is acceptable or unacceptable. ${ }^{170,172}$ Gilligan $(1982,1988)$ suggests that men and women effectively speak a different moral language and may sometimes use similar words to describe something that they assume is same but are subjected to mistranslation and/or misunderstanding. ${ }^{170,171}$ 
The findings of this study provide important insight into what men and women may understand as an unwanted sexual experience. However, there are several limitations of the study that should be considered. In order to lower bias in responses by non-victimized participants as a result of the exposure to questions asking about different types of sexual violence victimization we asked the open-ended question before a long series of victimization-related questions. It is likely that responses of victimized participants may be influenced by their personal experience. However, these results should not be interpreted as definitions of sexual victimization as participants were asked to define what they considered as an unwanted sexual experience not sexual victimization. Participants for this study were recruited from an undergraduate pool attending classes offered by a single academic department at a large Mid-Atlantic university and may not be generalizable to older students, those from other departments, or other regions.

Understanding differences in definitions of sexual violence is a critical first step in interpreting and assessing studies of gender and sexual victimization. Qualitative studies like the one described here may play a key role in advancing understanding of the unwanted sexual experiences encountered by young, college-attending men and women. Our findings suggest that it is important to understand how definitions of sexual assault (e.g. rape, molestation) influence what young men and women consider as sexual victimization. Also, our findings suggest that there exists a lack of awareness about different types of unwanted sexual experiences among those attending college and provides evidence of need for better awareness programs. This will not only help prevent sexual victimization but will also provide better estimates of sexual victimization among college population. Sexual victimization has both short- and long-term mental health consequences. Ultimately, to reduce sexual victimization among young adults, we must understand how young males and females interpret unwanted sexual experience. This 
study, though adds to the literature on definition of sexual victimization, it is critical to use more detailed qualitative approach for better understanding. 


\section{Table 4.1: Sample Characteristics}

\begin{tabular}{|c|c|c|c|c|}
\hline \multirow[t]{2}{*}{ Variable } & \multirow{2}{*}{$\begin{array}{r}\text { Victimized Males } \\
\text { Percentage (n) }\end{array}$} & \multirow{2}{*}{$\begin{array}{r}\text { Victimized Females } \\
\text { Percentage (n) }\end{array}$} & \multirow{2}{*}{$\begin{array}{r}\text { Non-Victimized Males } \\
\text { Percentage (n) }\end{array}$} & \multirow{2}{*}{$\begin{array}{r}\text { Non-Victimized Females } \\
\text { Percentage (n) }\end{array}$} \\
\hline & & & & \\
\hline \multicolumn{5}{|l|}{ Age Groups (in years) } \\
\hline $18-19$ & $53.95(41)$ & $71.62(212)$ & $64.71(44)$ & $73.13(98)$ \\
\hline $20-21$ & $34.21(26)$ & $22.97(68)$ & 27/94 (19) & $23.88(32)$ \\
\hline $22-23$ & $5.26(4)$ & $3.38(10)$ & $5.88(4)$ & $0(0)$ \\
\hline 24-25 & $2.63(2)$ & $0.68(2)$ & $0(0)$ & $2.24(3)$ \\
\hline 25+ & $3.95(3)$ & $1.35(4)$ & $1.47(1)$ & $0.75(1)$ \\
\hline \multicolumn{5}{|l|}{ Race } \\
\hline White & $96.05(73)$ & $94.59(280)$ & $92.65(63)$ & $89.55(120)$ \\
\hline African American & $1.32(1)$ & $2.03(6)$ & $1.47(1)$ & $2.99(4)$ \\
\hline Hispanic & $0(0)$ & $1.35(4)$ & $4.41(3)$ & $0.75(1)$ \\
\hline Asian or Pacific Islander & $1.32(1)$ & $0(0)$ & $1.47(1)$ & $0.75(1)$ \\
\hline Other & $1.32(1)$ & $2.03(6)$ & $0(0)$ & $5.97(0)$ \\
\hline \multicolumn{5}{|l|}{ Year in School } \\
\hline $1^{\text {st }}$ Year & $42.11(32)$ & $56.76(168)$ & $54.41(37)$ & $51.49(69)$ \\
\hline $2^{\text {nd }}$ Year & $32.89(25)$ & $25.00(74)$ & $19.12(13)$ & $29.85(40)$ \\
\hline $3^{\text {rd }}$ Year & $15.79(12)$ & $8.11(24)$ & $16.18(11)$ & $12.69(17)$ \\
\hline $4^{\text {th }}$ Year & $3.95(3)$ & $5.41(16)$ & $7.35(5)$ & $4.48(6)$ \\
\hline $5^{\text {th }}$ Year & $2.63(2)$ & $2.70(8)$ & $1.47(1)$ & $0.75(1)$ \\
\hline Graduate/Professional & $1.32(1)$ & $0(0)$ & $0(0)$ & $0.75(1)$ \\
\hline Other & $1.32(1)$ & $2.03(6)$ & $1.47(1)$ & $0(0)$ \\
\hline \multicolumn{5}{|l|}{ Current Relationship Status } \\
\hline Single & $75.00(57)$ & $67.57(200)$ & $58.82(40)$ & $52.24(70)$ \\
\hline Married/Cohabitating & $2.63(2)$ & $0.68(2)$ & $4.41(3)$ & $0(0)$ \\
\hline Committed/Engaged & $22.37(17)$ & $31.08(92)$ & $36.76(25)$ & $47.76(64)$ \\
\hline Divorced & $0(0)$ & $0.68(2)$ & $0(0)$ & $0(0)$ \\
\hline \multicolumn{5}{|l|}{ Sexual Orientation } \\
\hline Heterosexual & $97.37(74)$ & $95.95(284)$ & $95.59(65)$ & $98.51(132)$ \\
\hline Bisexual & $1.32(1)$ & $4.05(12)$ & $1.47(1)$ & $1.49(2)$ \\
\hline Gay/Lesbian & $1.32(1)$ & $0(0)$ & $1.47(1)$ & $0(0)$ \\
\hline Unsure & $0(0)$ & $0(0)$ & $1.47(1)$ & $0(0)$ \\
\hline
\end{tabular}




\section{Table 4.2: Types of Unwanted Sexual Experiences}

\begin{tabular}{|c|c|c|c|}
\hline Victimized Males (n) & Victimized Females (n) & Non-Victimized Males (n) & Non-Victimized Females (n) \\
\hline $\begin{array}{l}\text { Any sexual activity: (14) e.g. anything } \\
\text { sexual, some form of sex, anything that } \\
\text { involves sex, sex or sex related activity } \\
\text { Molestation: (2) e.g. molested against will, } \\
\text { molestation } \\
\text { Performing sexual act: (1) When one person } \\
\text { performs sexual actions when they do not } \\
\text { want to } \\
\text { Rape: (20) } \\
\text { Sex that is perpetrated by a male: (1) when } \\
\text { the girl does not want to do it, but the guy } \\
\text { proceeds anyway } \\
\text { Sex: (8) e.g. indicated unwanted sexual } \\
\text { experience as sex } \\
\text { Sexual advances: (1) sexual advances } \\
\text { Sexual comment: (2) } \\
\text { Sexual relation: (1) } \\
\text { Touching: (5) e.g. touching, kissing, sexual } \\
\text { touching/grabbing, } \\
\text { Visual: (1) e.g. sexual contact of any kind } \\
\text { being physical, visual, or verbal that is not } \\
\text { desired }\end{array}$ & $\begin{array}{l}\text { Any act of affection or infatuation that is } \\
\text { unwanted: (1) } \\
\text { Any sexual act that's given or received: (1) } \\
\text { e.g. An unwanted sexual experience is any } \\
\text { type of sex (oral, vaginal, anal) that you gave } \\
\text { or received that you did not want, when a } \\
\text { sexual act was perform or received, making } \\
\text { me do something sexually that i did not want } \\
\text { to do } \\
\text { Any sexual activity: (46) e.g. Any type of } \\
\text { sexual activity, unwanted sexual things } \\
\text { whether it be intercourse or not, anything } \\
\text { sexual, anything whether it be as little as } \\
\text { kissing. } \\
\text { Anything that involves private parts of } \\
\text { body: (1) e.g. anything involved w/ your } \\
\text { privates that you did not want to happen } \\
\text { Flirting: (2) e.g. someone hitting on me, } \\
\text { flirting } \\
\text { Molestation: (1) } \\
\text { Rape: (74) } \\
\text { Sex: (17) } \\
\text { Sexual aggression: (1) } \\
\text { Sexual Behavior: (1) e.g. forcing a sexual } \\
\text { behaviors onto someone who does not want } \\
\text { to do those behaviors } \\
\text { Sexual Harassment: (1) } \\
\text { Taking advantage: (1) e.g. Being sexually } \\
\text { taken advantage of } \\
\text { Touching: (14) e.g. touching or sexual } \\
\text { contact, Any unwanted touching, kissing, or } \\
\text { sexual activity, whether it be as little as } \\
\text { kissing. } \\
\text { Verbal abuse: (2) e.g. any verbal or physical } \\
\text { gesture promoting sexual behavior that you } \\
\text { did not want to engage in. }\end{array}$ & $\begin{array}{l}\text { Any sexual activity: (7) e.g. any sexual } \\
\text { experience, something sexual } \\
\text { Rape: (19) } \\
\text { Sex: (17) sexual intercourse } \\
\text { Sexual attention: (1) unwanted sexual } \\
\text { attention } \\
\text { Sexual Harassment: (2) } \\
\text { Touching: (7) e.g. any form of touching } \\
\text { whether it be sexual or not, any touching from } \\
\text { someone you don't want in private areas of } \\
\text { your body }\end{array}$ & $\begin{array}{l}\text { Any sexual act that's given or received: (3) } \\
\text { e.g. When someone makes you do something } \\
\text { sexual } \\
\text { Any sexual activity: (16) e.g. sex or any } \\
\text { sexual experience, any type of sexual act or } \\
\text { contact, anything sexual } \\
\text { Being looked at: (1) e.g. being looked at in a } \\
\text { way that I do not want } \\
\text { Foreplay: (1) } \\
\text { Rape: (32) } \\
\text { Revealing: (1) e.g. the forcing of or revealing } \\
\text { of a penis, vagina, or breasts into/onto any } \\
\text { part of the body that the other person does } \\
\text { not want } \\
\text { Sex: (10) } \\
\text { Sexual behavior: (3) e.g. any sexual } \\
\text { behavior, some kind of sexual behavior } \\
\text { Sexual Harassment: (2) } \\
\text { Taking advantage: (2) e.g. someone taking } \\
\text { advantage of you } \\
\text { Touching: (3) e.g. Any unwanted touching in } \\
\text { a sexual manner } \\
\text { Unwanted physical contact: (1) } \\
\text { Verbal abuse: (1) e.g. verbally subjected to } \\
\text { something that was uncomfortable relating to } \\
\text { sex }\end{array}$ \\
\hline
\end{tabular}




\section{Table 4.3: Description by Males and Females on Why the Sexual Experience was "Unwanted"}

\begin{tabular}{|c|c|c|c|}
\hline Victimized Males (n) & Victimized Females (n) & Non-Victimized Males (n) & Non-Victimized Females (n) \\
\hline $\begin{array}{l}\text { Against your will: (11) e.g. against person's } \\
\text { wish, any sexual contact that is not desired, } \\
\text { person raped or molested against their will } \\
\text { Because of the person (not partner): (3) e.g. } \\
\text { being with someone you don't feel comfortable } \\
\text { in that situation, a person you don't want to do } \\
\text { something with } \\
\text { Coercion: (6) peer pressure was also included } \\
\text { in this category e.g. One that you participate in } \\
\text { only because of external pressures, sex you did } \\
\text { not want but were pressured into it, doesn't } \\
\text { have to be physically forceful. Could use } \\
\text { begging } \\
\text { Even if verbally allow it: (1) e.g. When one } \\
\text { person performs sexual actions when they do } \\
\text { not want to even if they verbally allow it }\end{array}$ & $\begin{array}{l}\text { Against your will: (47) e.g. me not wanting to } \\
\text { have any sexual contact with another, but still } \\
\text { having it, Having someone do something to } \\
\text { you sexually against your will, If a person } \\
\text { doesn't want anything to happen, then nothing } \\
\text { needs to happen, or its unwanted, that occur to } \\
\text { someone who is unwilling } \\
\text { Coercion: (18) e.g. when someone is } \\
\text { pressured in some way to have sex when they } \\
\text { don't want to, emotionally forced into sex, } \\
\text { guilted into sex, etc, forced out of one partner } \\
\text { by persistence of the other partner, constant } \\
\text { peer pressure, A sexual experience that was } \\
\text { forced upon you either through pressure } \\
\text { Done under the influence: (17) e.g. occurs } \\
\text { under circumstances where one person is } \\
\text { severely intoxicated, does involve the use of } \\
\text { anything that would hinder your judgment, } \\
\text { such as alcohol, sex when you were under the } \\
\text { influence of drugs or alcohol, sexual act was } \\
\text { perform or received while intoxicated or } \\
\text { otherwise compromised } \\
\text { Encouraged into: (1) e.g. When you don't } \\
\text { want to be involved or when you are forced or } \\
\text { encouraged. }\end{array}$ & $\begin{array}{l}\text { Against your will: (7) e.g. against the will of } \\
\text { another, something you wouldn't want to do, } \\
\text { someone who does not want sex willingly } \\
\text { Because of the person (not partner): (2) e.g. } \\
\text { somebody that you really shouldn't be with, } \\
\text { person you don't want to have sex with } \\
\text { Both people don't know what is going on: (1) } \\
\text { e.g. both people do not want what is going on. } \\
\text { Coercion: (5) with and the other persuading to } \\
\text { do so, coercion of sex, mentally or physically, } \\
\text { excessively convinced to have sex or a sexual } \\
\text { act } \\
\text { Forced: (15) e.g. forcing yourself onto another } \\
\text { person in any form of touching whether it is } \\
\text { sexual or not, someone forcing themselves/you } \\
\text { into something that can be defined as sexual. } \\
\text { One partner not willing: (14) if one of the } \\
\text { partners does not wish to participate, when } \\
\text { someone doesn't want to have sex, but there } \\
\text { partner forces them without their consent, if } \\
\text { one of the partners does not which to } \\
\text { participate (usually a girl), to do something in a } \\
\text { sexual manner (hugging, fondling, kissing, etc.) } \\
\text { that is not accepted by the other partner } \\
\text { Said no: (1) anytime the word "no" is said } \\
\text { Not enjoyed: (1) e.g. sexual experience you did } \\
\text { not enjoy } \\
\text { Without Consent: (6) e.g. any sexual } \\
\text { experience without your consent, A sexual } \\
\text { experience performed by either partner without } \\
\text { consent of the other, if it is not agreed upon }\end{array}$ & $\begin{array}{l}\text { Against your will: (19) e.g. having sex against } \\
\text { your wishes, anything you are not willing to } \\
\text { do, unwilling had to perform } \\
\text { Not Enjoyed: (1) an experience that you have } \\
\text { with a partner that you did not enjoy or want in } \\
\text { a sexual manner } \\
\text { Coercion: (6) e.g. Pressured into doing things } \\
\text { you didn't want to do, when a person pressures } \\
\text { you into it, persistent, aggressive and without } \\
\text { consent } \\
\text { Done under the influence: (3) taking } \\
\text { advantage of you while you are under the } \\
\text { influence of drugs or alcohol } \\
\text { Forced: (32) e.g. being forced to have sex, } \\
\text { Being forced to act in sexual experiences, } \\
\text { Being forced to do a sexually deed } \\
\text { With someone you don't want to: (2) e.g. an } \\
\text { unwanted sexual experience is having sex or } \\
\text { any other sexual activity with someone whom } \\
\text { you do not want to be engaged in } \\
\text { Inappropriate: (1) any sexual behavior that } \\
\text { one partner finds inappropriate } \\
\text { Not ready for: (1) e.g. something you are not } \\
\text { ready for, you don't want to do. } \\
\text { One partner not willing: (5) e.g. anything } \\
\text { sexual that is not wanted by one or both people, } \\
\text { Either the male or female in the relationship } \\
\text { not wanting to have a sexual experience occur } \\
\text { Said no: (8) e.g. They tell their partner to stop } \\
\text { but they just continue on, Someone forcing you } \\
\text { to have sex with them after you've said no } \\
\text { Unwelcomed: (1) e.g. a non welcoming action } \\
\text { towards another person } \\
\text { Without consent: (10) e.g. Sexual act that was } \\
\text { conducted without my approval, when one } \\
\text { person is not consenting }\end{array}$ \\
\hline
\end{tabular}




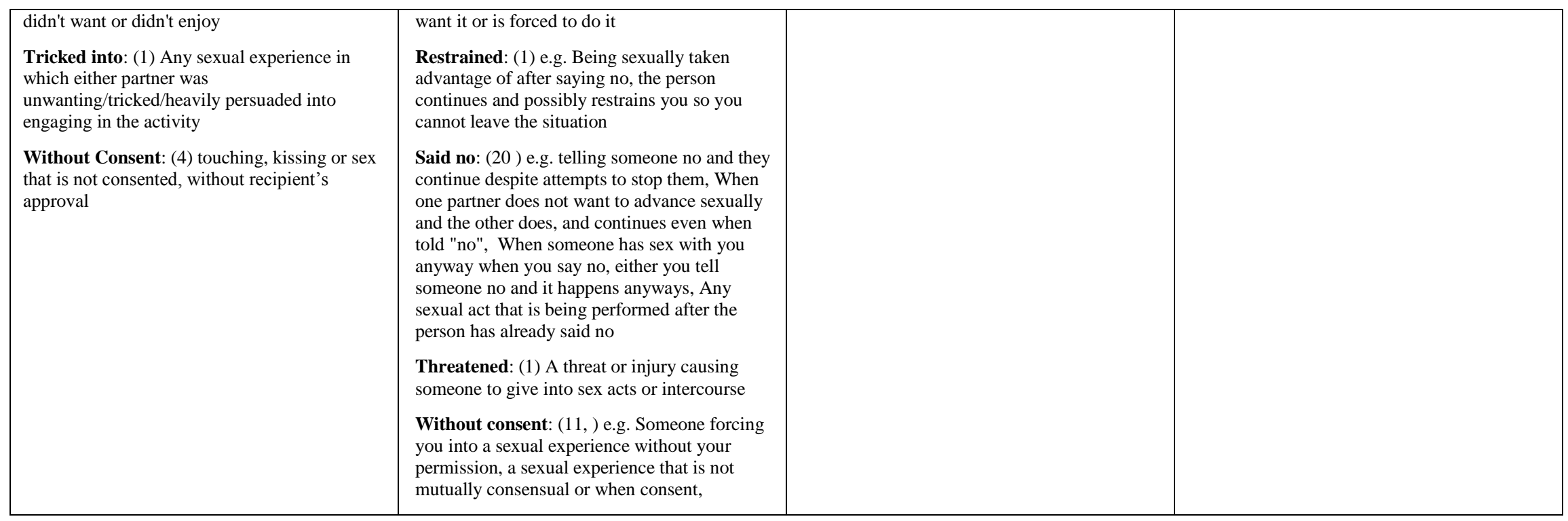


Table 4.4: Emotions or Feelings Expressed When Defining Unwanted Sexual Experience

\begin{tabular}{|c|c|c|c|}
\hline Victimized Males (n) & Victimized Females (n) & Non-Victimized Males (n) & Non-Victimized Females (n) \\
\hline $\begin{array}{l}\text { Disgusted: (1) } \\
\text { Sad: (1) } \\
\text { Uncomfortable: (3) } \\
\text { Regret: (4) }\end{array}$ & $\begin{array}{l}\text { Angry: (1) } \\
\text { Anything that will not make you happy: (2) } \\
\text { Causes unwanted feelings: (1) } \\
\text { Difficult: (1) } \\
\text { Feel used: (2) } \\
\text { Harmful: (1) } \\
\text { Painful: (5) } \\
\text { Sad: (1) } \\
\text { Scary: (6) } \\
\text { Uncomfortable: (11) } \\
\text { Unsatisfying: (1) } \\
\text { Violated: (1) } \\
\text { Regret: (7) }\end{array}$ & $\begin{array}{l}\text { Uncomfortable: (1) } \\
\text { Regret: (3) }\end{array}$ & $\begin{array}{l}\text { Horrible: (2) } \\
\text { Hurt: (1) } \\
\text { Scarring: (1) } \\
\text { Uncomfortable: (6) } \\
\text { Regret: (1) }\end{array}$ \\
\hline
\end{tabular}


Figure 4.1: Conceptual Model of Definition of Unwanted Sexual Experience

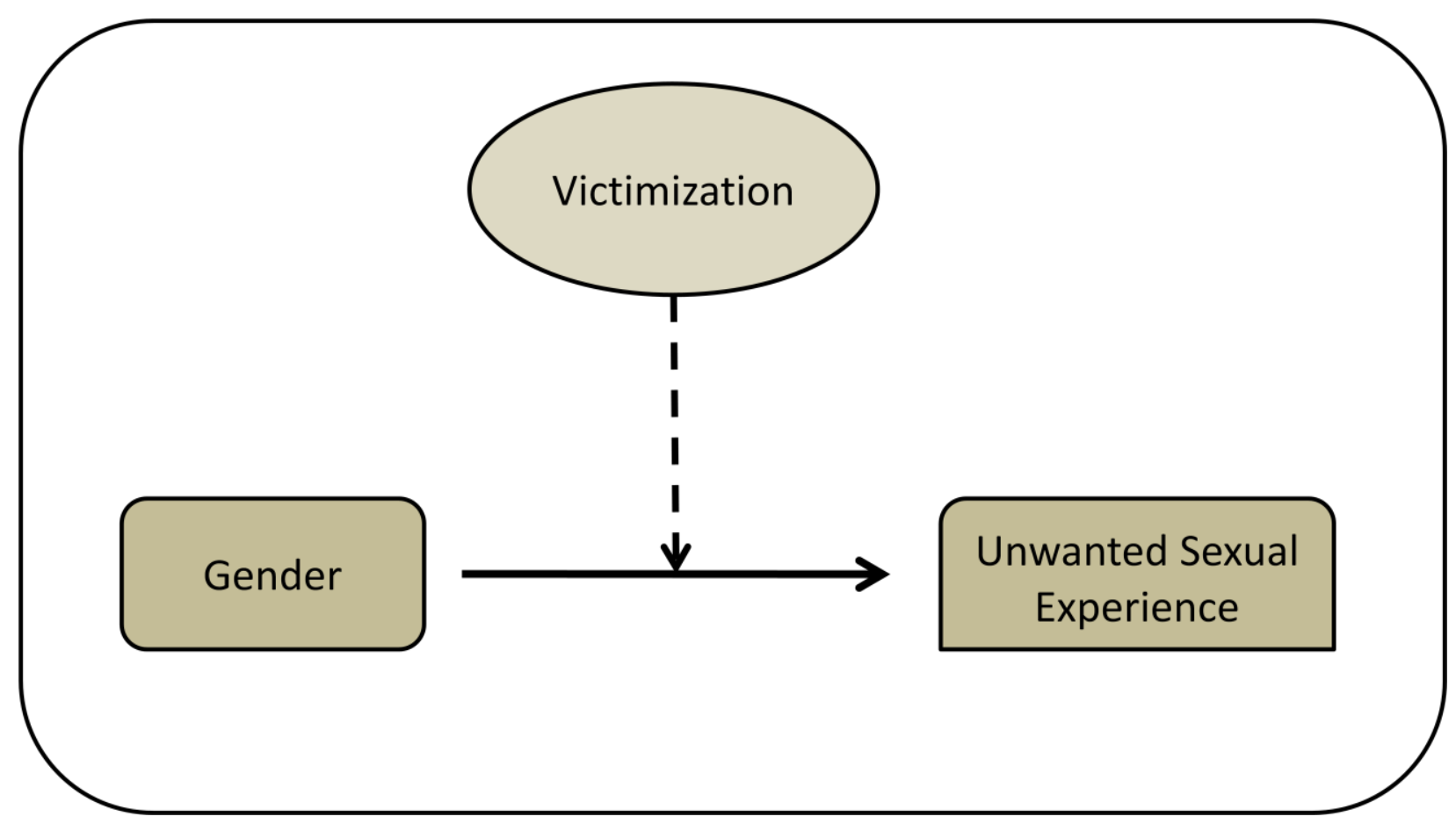




\section{Figure 4.2: Organization of Definitions of Unwanted Sexual Experience}

\begin{tabular}{|c|c|c|}
\hline \multicolumn{3}{|c|}{ Concepts/Themes } \\
\hline Any sexual activity & Against your will & Angry \\
\hline Molestation & Coercion & Anything that will not make you happy \\
\hline Performing sexual act & Done under the influence & Causes unwanted feelings \\
\hline Rape & Encouraged into & Difficult \\
\hline Sex that is perpetrated by a male and female is the victim & Feel obligated to do so & Feel used \\
\hline Sex & Forced & Harmful \\
\hline Sexual advances & One partner not willing & Painful \\
\hline Sexual comment & Restrained & Sad \\
\hline Sexual relation & Said No & Scary \\
\hline Touching & Threatened & Unsatisfying \\
\hline Visual & Without consent & Violated \\
\hline Sexual attention & Because of the person & Regret \\
\hline Sexual harassment & Even if verbally allow it & Uncomfortable \\
\hline Any act of affection or infatuation & Mistake & Disgusted \\
\hline Any sexual activity & Not enjoyed & Horrible \\
\hline Anything that involves private parts of body & Tricked into & Hurt \\
\hline Flirting & Both people don't know what is going on & Scarring \\
\hline Sexual aggression & With someone you don't want to & \\
\hline Verbal abuse & Inappropriate & \\
\hline Taking advantage & Not ready for & \\
\hline Being looked at & Unwelcomed & \\
\hline Revealing & & \\
\hline & & 1 \\
\hline Type of Victimization & Why Unwanted & Emotions/Feelings \\
\hline Unwal & d Sexual Experience & \\
\hline
\end{tabular}


Chapter 5 


\section{Chapter 5}

\subsection{Summary}

Sexual assault can refer to any form of forcible or non-forcible sexual act where consent was not obtained or freely given. Previous studies have shown that victims of sexual assault are likely to experience adverse psychological and physical consequences. ${ }^{71,127}$ Existing studies have documented the prevalence and long-term consequences of sexual victimization among females. However, the current understanding of male sexual assault is limited. ${ }^{39,155,173}$ In comparison to female victims, there is a relative paucity of literature examining the characteristics and adverse health outcomes associated with male sexual assault, ${ }^{4,56,126}$ as well as long-term consequences. $^{16,17}$ Current understanding of the physical and psychological consequences of male sexual assault is based primarily on studies of female victims or select clinical or demographic populations. For example, the few studies that have considered the impact of sexual violence on males have been primarily focused on childhood sexual abuse or among adult males in sexually segregated institutions such as prison. ${ }^{67,79,174}$ In general, empirical studies of male sexual assault have drawn data from small clinical samples and their findings may be limited in their generalizability. ${ }^{19,28,30,38}$

The main objective of this study was to enhance our understanding of male sexual assault through results obtained from three related projects. Current estimates of the rate of male sexual assault in the general population have been primarily based on results from self-reported surveys

or clinical assessments. ${ }^{4,11}$ Therefore, in the first study, an epidemiological profile was developed of male sexual assault describing victim-, offender-, arrestee- and incident-related characteristics. The scant literature on psychological effects of male sexual assault suggests that sexual victimization can be associated with a greater prevalence of risk behaviors (such as 
alcohol use, substance abuse, and smoking) and victimized males may be at greater risk of perpetration of violence-related behavior. ${ }^{29,47,126}$ To identify associations between interpersonal and intimate partner violence and sexual assault, the relationships between sexual victimization and aggressive or high risk behaviors among a sample of college-attending males and females were examined. Finally, the term "sexual assault" has been previously used to describe a broad range of different categories of victimization, ranging from unwanted kissing to non-consensual sex. Given prevailing societal beliefs and notions of traditional gender roles (i.e., that men cannot be the victims of sexual assault or that men are less affected by sexual assault than women $),{ }^{8-10}$ because men and women may differ in their understanding of victimization, and the strong likelihood that victimization may affect definitions of sexual violence, the final study sought to better understand how definitions of sexual victimization differ between men and women and those who have and have not been victimized.

The accurate assessment of the incidence of sexual assault of men is a challenging task. Researchers in the past have estimated the prevalence of sexual violence among males using data obtained from nationally administered surveys or using clinically-based samples. However, selfreport survey data are subject to selection and recall bias and clinically-based samples are dependent on access to and use of clinical services. It is possible that other data sources, such as criminological records, could contribute substantially to the existing knowledge of the incidence and characteristics of male sexual assault. To explore this possibility, data from the National Incident Based Reporting System (NIBRS) was used to construct a 5-year epidemiological profile of male sexual assault. In addition to characteristics of the incident, such as relationships with the perpetrator and severity of injury, this study provided estimates of age-, race-, and ethnicity-specific rates of male sexual assault. Estimates calculated from NIBRS data (chapter 2) 
suggest that the majority of male sexual assault victims for all 5 years were under the age of 19 years (approximately 80\% each year) and white (range: 80.02\%-83.90\%). A significant change over time was observed for age groups 0-9, 10-19, 20-29, 30-39, 40-49, 50-59, and 70-79. Racespecific comparisons show that the rate of sexual assault was higher among African American males for all age groups as compared to White males. The time trend analyses for White males were similar to those observed among all males and no race group by the time interaction for adult African American males was observed. A limited number of previous studies have examined the perpetrator characteristics of male sexual assault using clinical samples. ${ }^{19,21}$ In this study, offender and arrestee characteristics were examined. The majority of offenders were male, white, and under the age of 39 years. Over the 5-year period, an average of less than one arrest per incident was made and there were few demographic differences between the arrestee and reported offender. The characteristics of sexual assault were also examined. Forcible sexual assaults, which included rape, sodomy, sexual assault with an object, and forcible fondling, were the highest reported incidents of male sexual assault. The majority of these sexual assault incidents took place at a residential location and did not involve use of a weapon. Findings from emergency room reports and clinical studies of male sexual assault suggest that a typical male victim present with some type of physical injuries. ${ }^{8,28,30}$ Results suggest that the majority of the victims in all age groups did not receive any injuries during the assault. Based on analyses of NIBRS data, there is evidence that criminological data may provide additional information on reported incidents of male sexual assault, including information on incidents not routinely included in self-report survey data.

Sexual victimization is often associated with psychological morbidity such as depression, anxiety, suicidal behavior, and substance abuse. ${ }^{7,12,15}$ Previous studies have suggested that 
female victims may be more likely to express internalized emotions ${ }^{46,70}$ and males may be more likely to express externalized behaviors. ${ }^{37,68}$ However, the associations between sexual violence victimization and externalizing behaviors among males are not well understood.

Existing literature suggests that a significant number of college-attending males may experience unwanted sexual contact. Despite the possibility of a high prevalence of sexual assault victimization among young adults, few studies have examined gender differences in psychopathology among victimized males and females. ${ }^{72,92,126}$ The main objectives of the second project in this study were to explore gender differences among multiple categories of sexual assault victimization and the associations between victimization and adverse mental health outcomes among students attending a large Mid-Atlantic U.S. university. To obtain data for this project, a self-administered questionnaire using standardized measures was used to collect information on social, behavioral, and psychological characteristics.

Results from this project are in concert with existing literature, showing that the prevalence of sexual victimization was higher among females than males. The prevalence of current depression was higher among victimized males and females when compared to non-victimized males and females, respectively. Also consistent with previous studies, results from this project identified a higher prevalence of risk behaviors such as tobacco, alcohol, and cocaine use and heavy episodic drinking among male participants when compared to females that were independent of victimization. ${ }^{143,144}$ In comparison with non-victimized males, a higher prevalence of physical, emotional, and sexual violence perpetration were observed among victimized males. Statistically significant associations between severe sexual violence victimization (such as attempted rape, sexual assault with an object, or completed rape) and 
perpetration of interpersonal and intimate partner violence (physical, emotional, and sexual) were also observed among victimized males.

Adult males and females who are sexually assaulted commonly report symptoms of depression, suicide ideation, and deliberate self-harm (DSH). ${ }^{41,49,50,63}$ Results from the survey of 720 college-attending males $(n=194)$ and females $(n=526)$ identified no significant differences in the prevalence of suicidal behaviors among victimized males and females (chapter 3). The overall prevalence of DSH was lower among males and females than has been reported in previous studies. ${ }^{46,63}$ It is possible that the failure to identify gender differences in DSH may have resulted from low statistical power (Appendix C). Findings from this project emphasize the need for better understanding of male sexual assault and more definitive research on the emotional consequences of unwanted sexual experiences, especially among young adults.

The third project in this study was designed to explore gender- and victimization-related differences in definitions of an unwanted sexual experience. Using qualitative methods, three main categories of unwanted sexual experience emerged: (1) type of unwanted sexual experience; (2) why it was unwanted; and (3) emotions or feelings expressed towards the unwanted sexual experience. Regardless of the history of victimization, a broad range of types of unwanted sexual activities, from flirting to forcible sexual intercourse, were reported by both male and female participants. When describing why the sexual experience was unwanted, a majority of participants indicated that it was against their will or was not desired. Victimized males and females also indicated that a sexual experience was unwanted when a partner forced a sexual act or used coercive tactics. A large number of victimized participants (both males and females) and non-victimized females also identified the role of their own excessive use of alcohol in their descriptions of an unwanted sexual experience. Qualitative studies like the one 
described here may have a key role to play in advancing understanding of response to, and definitions of, unwanted sexual experiences shared by men and women attending college.

In summary, despite the emergence of a substantial literature on sexual assault, there has been comparatively little focus on male sexual assault victimization, and a poor understanding of the potential impact of sexual assault on the physical and mental health of male victims. Young men appear to be at particular high risk for sexual assault, and many may experience a sexual assault during college.

\subsection{Significance}

The long-term goal of this study was to increase knowledge about male sexual violence victimization, which, in turn, will help to inform the development of intervention strategies and prevention programs. The significance of this study is threefold. First, the characteristics of male sexual assault, especially among adult males, are not well studied. In the first project of this study, NIBRS data were used to estimate the national rate of male sexual assault and explore characteristics of sexual assault incidents, victims, and perpetrators. To enhance understanding of both the physical and psychological impacts of male sexual assault, the second project collected original self-reported data from a sample of undergraduate university students to calculate the prevalence of sexual violence and correlated risk behaviors (such as heavy episodic drinking, substance abuse, and violence-related behavior) among males and females. Finally, qualitative data collected from the second project was used to explore gender differences in definitions of sexual assault and identified three separate themes. Information from this study can be used to enhance understanding of how males and females may differ in their understanding of an unwanted sexual experience 
Developmental theories of late adolescence and early adulthood have emphasized the importance of identity formation and interpersonal relationships. ${ }^{175}$ For young adults, the development of interpersonal and intimate relationships requires significant levels of trust, self-acceptance, selfconfidence, independence, and self-awareness. ${ }^{175}$ Given the critical importance of identity and interpersonal relationships, the psychological impact of sexual assault among college-aged males should be addressed to minimize the impact on individual mental health and interpersonal relationships. Poor psychological health, increased likelihood of risk behaviors, and suicide ideation are not uncommon among victims of sexual assault. Given evidence that men can be victims of sexual assault on college campuses, a variety of intervention strategies should be considered for implementation by colleges and universities. Although most college prevention programs target female students, research on male sexual assault supports the need for programs responsive to the needs of both genders. To ensure that victims receive appropriate care, it is important to educate health services and university authorities about the estimated prevalence and correlates of male sexual assault

From a public health perspective, this research estimated the prevalence of sexual violence and associated risk factors and predictors associated with sexual violence and addressed the injury and violence prevention aspect of Healthy People 2010. ${ }^{176}$ Estimating the extent of adult male sexual assault in the general population has particular relevance to Objectives 15-34 (reduce the rate of physical assault by current or former intimate partners), 15-35 (reduce the annual rate of rape or attempted rape), and 15-36 (reduce sexual assault other than rape). ${ }^{176}$

\subsection{Strengths and Weaknesses}

This study highlights the importance of using multiple data sources to enhance the understanding of male sexual assault in the general population. To our knowledge, this is the first study to use 
NIBRS data to generate a national epidemiological profile of male sexual assault. Unlike population-based survey data and clinical studies, NIBRS data includes details about a large number of sexual assault victims, providing information on the characteristics of both perpetrators and the incident. The multi-level nature of NIBRS data was ideal for exploring characteristics of male sexual assault. Importantly, the NIBRS is publically available for research and provides a useful data source which can assist in reducing the burden of violencerelated public health problems such as sexual assault.

Consistent with reports from existing research, the current study has identified psychological and interpersonal correlates of sexual assault and gender-based differences in outcomes. Based on the estimated prevalence reported by this and other studies, sexual victimization among collegeaged students continues to be a significant problem. Unlike previous studies that have used less expansive definitions of sexual assault (e.g. attempted and completed rape), this study used the Sexual Experience Survey (SES), which includes a wide range of categories of sexual victimization to understand the associations between victimization and adverse outcomes among college-aged students.

While the quantitative projects in this study have provided information on the extent of the problem and associations between sexual victimization and adverse health outcomes, the qualitative project was used to further elucidate how males and females may define an unwanted sexual experience. Considering reports of a higher prevalence of sexual victimization among young adults, it is important to understand how males and females may differ in their understanding of an unwanted sexual experience. Study results highlighted some important similarities and some key differences in how young males and females define an unwanted sexual experience and how having been a victim may affect their understanding. 
It is important to note some key limitations of this project, as well. The NIBRS data collects information on sex offenses from state law enforcement agencies which voluntarily submit data to NIBRS. As reported in Chapter 2, only 24 of the 50 U.S. states were represented in our study. The use of such a small number of states may limit generalizability to non-participating states and regions. Further, this study did not account for differences in response and reporting that may be associated with organizational characteristics. Future studies should account for these types of differences. In the available NIBRS data, $40 \%$ of the information on ethnicity of the victims was missing which limited our ability to calculate ethnicity-specific rates of male sexual assault. Also, because NIBRS is derived from official police records, the reliability of injury data is unknown. Future studies comparing police reports of injury to self-reported victimization and clinical assessments are recommended. Additionally, differences in definitions of sexual assault used by NIBRS and previous studies may limit the direct comparison of study findings to others.

The second study in this project was designed to more fully understand gender differences in the psychological consequences associated with sexual victimization using a self-administered questionnaire and was also subject to important limitations. Similar to other studies of sexual assault in college populations, the generalizability of the current study may be limited by several factors. The current study obtained data from a sample of undergraduate students taking a select number of classes in the Department of Psychology at a single university in the Mid-Atlantic region of the U.S. Thus, the associations identified in analyses of these data may be limited to this population and may not be generalizable to older students, those in other departments, students at other universities, or those not attending college. Because the current study used a convenience sample, participants self-selected into the online study and may have been 
influenced in their decision by some unobserved and related factor. Therefore, it is possible that an unknown degree of selection bias may have influenced the reported proportions and associations. Studies that obtain samples from other populations or using different inclusion criteria may not yield similar results. Additionally, participation was limited to students who were enrolled in a small number (four) of undergraduate psychology classes.

Based on the initial assessment of the prevalence of sexual assault among males and females, emr sample size calculations (assuming medium effect size) resulted in the identification of a sample of 300 males and 300 females to obtain sufficient statistical power. Even though several methods (e.g., flyers, in-class introduction of the study) were used to increase participation, it was more difficult to recruit men than women. At the conclusion of the study and comprehensive data cleaning (see Appendix $\mathrm{C}$ for details on data cleaning procedures) the analytic sample size for males was limited to 194 cases. This sample size provided adequate statistical power for most of the proposed analyses but lower statistical power for others (see Appendix $\mathrm{C}$ for details). Also, approximately $8 \%$ of the data were missing information on sexual victimization and were excluded from all analyses. Because this study used crosssectional data, it was not possible to infer temporal ordering or causation.

One limitation associated with the use of qualitative methods is inability inappropriateness to generalize to other populations. Finally, the study was exploratory in nature and data was coded by only two researchers.

\subsection{Future Research}

The findings of this study highlight the need for a better understanding of male sexual assault. Priorities for future studies might include the epidemiology of male sexual assault, more nuanced 
understandings of the emotional, physical, and psychological consequences of sexual violence victimization, and the development of targeted interventions informed by understandings of gender differences in definitions of sexual assault. Future studies are urged to use available data sources from multiple sources to enhance understanding of comparatively rare events such as male sexual assault. Further empirical study of the psychological consequences of unwanted sexual experiences for both genders is warranted. Since available literature suggests a higher prevalence of sexual victimization among the college population, there is a need for sustained efforts to develop and disseminate educational campaigns to increase awareness among younger students. University-sponsored sexual assault programs, traditionally designed to serve female victims, are urged to include males. Study results identified a significant association between sexual victimization and violence-related behaviors among victimized males. Prevention specialists and those working with victimized populations should consider the possibility of sexual violence victimization among those engaging in violent or destructive behaviors. However, additional research is needed to confirm these findings and to identify appropriate opportunities for intervention.

\subsection{Conclusion}

The findings of this study can be used to extend our understanding of the extent and nature of sexual assault among males. Results indicated that despite a higher rate of sexual assault among young males, sexual assault incidents were reported by men of all age groups. Using NIBRS data for multiple years, significant age- and race-specific time-trends were observed among males. The results of the current study support previous findings that sexual victimization is associated with a range of health risk behaviors and negative psychological consequences among males. In addition, this study adds new information about the associations between sexual 
victimization and aggressive behaviors among males, including the co-occurrence of risk behavior such as alcohol and substance abuse. Consistent with previous literature, significant symptoms of depression among victimized males and females were observed. The findings of qualitative study also highlighted some important differences and similarities in the description of unwanted sexual experience among males and females. In comparisons to women, relatively little work has been done in the area of male sexual assault. For better understanding of different aspects of male sexual assault, more comprehensive and multidisciplinary approaches are needed. This study demonstrated the importance of investigating male sexual assault using various data sources. Strong preliminary evidence has emerged from this study that supports and extends previous research and establishes the need for future in-depth investigations of the behavioral and psychological consequences of sexual violence victimization. 
Appendix A 


\section{Appendix A}

\section{Survey: Sexual assault victimization and associated risk behaviors in college students Introduction}

This survey is about physical and mental health, sexual violence, and health-related behaviors (including suicide Ideation and self-injurious behavior). It has been developed to understand how various health behaviors and experiences may affect your overall health. The information you provide will help us better understand the relationships between past experiences and current health status.

A brief introduction is provided at the beginning of each section. Please read all the options carefully before answering.

\section{$\underline{\text { The option of N/A = Do not wish to respond }}$}

Thank you very much for your help.

\section{General Health (GH)}

The following set of questions asks about your general health.

1. GH01: In general, would you say your health is:
Excellent
Very Good
Good
Fair
Poor
N/A

2. GH02: In general, how satisfied are you with your life?
Very Satisfied
Satisfied
Dissatisfied
Very Dissatisfied
N/A

3. GH03: Now thinking about your physical health, which includes physical illness and injury, for how many days during the past 30 days was your physical health not good?
None
1-2 days
3-5 days
6-9 days
10-19 days
20-29 days
All 30 days
N/A

4. GH04: Now thinking about your mental health, which includes stress, depression, and problems with emotions, for how many days during the past 30 days was your mental health not good?
None
1-2 days 3-5 days
6-9 days
10-19 days 20-29 days
All 30 days
N/A

5. During the past 30 days, how many days did you use:

\begin{tabular}{|c|c|c|c|c|c|c|c|}
\hline $\begin{array}{l}\text { GH05: Tobacco } \\
\text { (cigarettes and/or } \\
\text { smokeless) }\end{array}$ & Never used & $\begin{array}{l}\text { Have used, but not } \\
\text { in last } 30 \text { days }\end{array}$ & 1-2 days & 3-5 days & 6-9 days & 10-19 days & 20-30 days \\
\hline GH06: Alcohol & Never used & $\begin{array}{l}\text { Have used, but not } \\
\text { in last } 30 \text { days }\end{array}$ & 1-2 days & 3-5 days & 6-9 days & 10-19 days & 20-30 days \\
\hline
\end{tabular}




\begin{tabular}{|c|c|c|c|c|c|c|c|c|}
\hline GH07: Marijuana & Never used & $\begin{array}{l}\text { Have used, but not } \\
\text { in last } 30 \text { days }\end{array}$ & 1-2 days & 3-5 days & 6-9 days & 10-19 days & 20-30 days & N/A \\
\hline GH08: Cocaine & Never used & $\begin{array}{c}\text { Have used, but not } \\
\text { in last } 30 \text { days }\end{array}$ & 1-2 days & 3-5 days & 6-9 days & 10-19 days & 20-30 days & N/A \\
\hline $\begin{array}{l}\text { GH09: Other } \\
\text { drugs }\end{array}$ & Never used & $\begin{array}{l}\text { Have used, but not } \\
\text { in last } 30 \text { days }\end{array}$ & 1-2 days & 3-5 days & 6-9 days & 10-19 days & 20-30 days & N/A \\
\hline
\end{tabular}

6. GH10: One drink is equivalent to a 12-ounce beer, a 5-ounce glass of wine, or a drink with one shot of liquor. During the past 30 days, on the days when you drank, about how many drinks did you drink on the average?
None
1-2 drinks
3-5 drinks
6-9 drinks
10 or more drinks
N/A

7. GH11: Considering all types of alcoholic beverages, how many times during the past 30 days did you have $\mathbf{5}$ or more drinks on an occasion?

None $\quad 1-2$ times $\quad 3-5$ times 6-9 times $\quad 10-19$ times $\quad 20-29$ times N/A

\section{Contraceptive Use (CU)}

The following set of questions asks about contraceptive use.

8. Did you or your partner use a condom the last time you had:
I. CU01: Oral sex?
Yes
No
Don't Know
Never did this sexual activity
N/A
II. CU02: Vaginal
Yes
No
Don't Know
Never did this sexual activity
N/A intercourse?
III. CU03: Anal intercourse?
Yes

No
Don't Know Never did this sexual activity
N/A

9. CU04: If you had vaginal intercourse, what method did you or your partner use to prevent pregnancy or sexually transmitted diseases (STD) the last time? (Select all that apply)

Birth control pills

Depo Provera (shots)

Norplant (implant)
Spermicide

Fertility awareness (calendar, basal body temp)

Withdrawal

CU05: If you had vaginal intercourse, what method did you or your partner use to prevent pregnancy or STD the last time? (Select all that apply)
Condoms (male or female)
Other method
Diaphragm/Cervical cap/Sponge
Nothing 
Have not had vaginal intercourse

Intra-Uterine Device (IUD)

N/A

10. How much of the following factors do you think influence your decision to engage in sexual behavior (Select all that apply)

\begin{tabular}{|c|c|c|c|}
\hline CU06: Personal Choice & None & Somewhat & A great deal \\
\hline CU07: What date/partner expects of me & None & Somewhat & A great deal \\
\hline CU08: What friends expect of me & None & Somewhat & A great deal \\
\hline CU09: What parents expect of me & None & Somewhat & A great deal \\
\hline CU10: What society expects of me & None & Somewhat & A great deal \\
\hline
\end{tabular}

11. CU11: Within the last school year, with how many partners, if any, have you had sex (oral, vaginal, or anal)?

None

1-2 partners

3-4 partners

5 or more partners

N/A

\section{Sexual Violence (SV)}

The following questions ask about any unwanted sexual experience you may have had at some point in your lifetime. An unwanted sexual experience is one that you did not want to do, but resulted in sexual activity anyway. The unwanted sexual experience could have been anything from an unwanted kiss to unwanted sexual intercourse. Please read each statement and check the appropriate response depending on whether any of these things happened to you. If you answer YES to any question please answer the question asking if this person was a male or female.

12. SV01: Have you ever given into sex play (fondling, kissing, or petting, but not intercourse) when you didn't want to because you were overwhelmed by the person/partner's continual argument and pressure?

$$
\begin{array}{lll}
\text { Yes No N } & \text { No }
\end{array}
$$

13. SV02: If yes, was this person:
Male
Female
N/A 
14. SV03: Have you ever had sex play (fondling, kissing, or petting, but not intercourse) when you didn't want to because the person/partner used his/her position of authority (boss, teacher, camp counselor, supervisor, family member etc.) to make you?
Yes
No
N/A

15. SV04: If yes, was this person:
Male
Female
N/A

16. SV05: Have you ever had sex play (fondling, kissing, or petting, but not intercourse) when you didn't want to because the person/partner gave you alcohol or drugs?
Yes
No
N/A

17. SV06: If yes, was this person:

Male

Female

N/A

18. SV07: Have you ever had sex play (fondling, kissing, or petting, but not intercourse) when you didn't want to because you consumed too much alcohol or drugs willingly?
Yes
No
N/A

19. SV08: If yes, was this person:
Male
Female
N/A

20. SV09: Have you ever had sex play (fondling, kissing, or petting, but not intercourse) when you didn't want to because the person/partner threatened or used some degree of physical force (twisting your arm, holding you down, etc.) to make you?
Yes
No
N/A

21. SV10: If yes, was this person:

$\begin{array}{lll}\text { Male Female N/A } & \text { F }\end{array}$

22. SV11: Have you ever given into sex play (fondling, kissing, or petting, but not intercourse) because you were concerned about what others might think of you if you did not give in or because you thought others expected you to?

Yes

No

N/A

23. SV12: Have you ever had a person/partner attempted sexual intercourse (attempted vaginal or anal intercourse between persons regardless of gender) when you didn't want to because you were overwhelmed by the person/partner's continual argument and pressure?

Yes

No 
24. SV13: If yes, was this person:
Male
Female
N/A

25. Have you ever had a person/partner attempted sexual intercourse (attempted vaginal or anal intercourse between persons regardless of gender) when you didn't want to by using his/her position of authority (boss, teacher, camp counselor, supervisor, family member etc.) to make you, but intercourse did not occur?
Yes
No
N/A

If yes, was this person:

Male

Female

N/A

26. Have you ever had a person/partner attempted sexual intercourse (attempted vaginal or anal intercourse between persons regardless of gender) when you didn't want to by giving you alcohol or drugs, but intercourse did not occur?
Yes
No
N/A

If yes, was this person:

Male

Female

N/A

27. Have you ever had a person/partner attempted sexual intercourse (attempted vaginal or anal intercourse between persons regardless of gender) when you didn't want to because you consumed too much alcohol willingly, but intercourse did not occur?
Yes
No
N/A

If yes, was this person:

Male

Female

N/A

28. Have you ever had a person/partner attempted sexual intercourse (attempted vaginal or anal intercourse between persons regardless of gender) when you didn't want to by threatening or using some degree of force (twisting your arm, holding you down, etc.), but intercourse did not occur?
Yes
No
N/A

If yes, was this person:

Male

Female

N/A

29. Have you ever had a person/partner attempted sexual intercourse (attempted vaginal or anal intercourse between persons regardless of gender) but intercourse did not occur because you were concerned about what others might think of you if you did not given in or because you thought others expected you to?
Yes
No
N/A 
30. Have you ever participated in sex acts (oral sex and/or anal or vaginal penetration by object other than the penis) when you didn't want to because you were overwhelmed by the person/partner's continual argument and pressure?
Yes
No
N/A

If yes, was this person:

Male

Female

N/A

31. Have you ever participated in sex acts (oral sex and/or anal or vaginal penetration by object other than the penis s) when you didn't want to by using his/her position of authority (boss, teacher, camp counselor, supervisor, family member etc.) to make you?
Yes
No
N/A

If yes, was this person:

Male

Female

N/A

32. Have you ever participated in sex acts (oral sex and/or anal or vaginal penetration by object other than the penis) when you didn't want to because the person/partner gave you alcohol or drugs?
Yes
No
N/A

If yes, was this person:

Male Female N/A

33. Have you ever participated in sex acts (oral sex and/or anal or vaginal penetration by object other than the penis) when you didn't want to because you consumed too much alcohol or drugs willingly?
Yes
No
N/A

If yes, was this person:

Male

Female

N/A

34. Have you ever participated in sex acts (oral sex and/or anal or vaginal penetration by object other than the penis) when you didn't want to because another person/partner threatened or used some degree of physical force (twisting your arm, holding you down, etc.) to make you?
Yes
No
N/A

\section{If yes, was this person:}
Male
Female
N/A

35. Have you ever participated in sex acts (oral sex and/or anal or vaginal penetration by object other than the penis) because you were concerned about what others might think of you if you did not give in or because you thought others expected you to?
Yes
No
N/A 
36. Have you ever had sexual intercourse (vaginal or anal intercourse between persons regardless of gender) when you didn't want to because you were overwhelmed by the person/partner's continual argument and pressure?
Yes
No
N/A

If yes, was this person:

Male

Female

N/A

37. Have you ever had sexual intercourse (vaginal or anal intercourse between persons regardless of gender) when you didn't want to because a person/partner used his/her position of authority (boss, teacher, camp counselor, supervisor, family member etc.) to make you?
Yes
No
N/A

If yes, was this person:

Male

Female

N/A

38. Have you ever had sexual intercourse (vaginal or anal intercourse between persons regardless of gender) when you didn't want to because the person/partner gave you alcohol or drugs?
Yes
No
N/A

If yes, was this person:
Male
Female
N/A

39. Have you ever had sexual intercourse (vaginal or anal intercourse between persons regardless of gender) when you didn't want to because you consumed too much alcohol or drugs willingly?

$\begin{array}{lll}\text { Yes No N/A } & \text { No }\end{array}$

40. Have you ever had sexual intercourse (vaginal or anal intercourse between persons regardless of gender) when you didn't want to because another person/partner threatened or used some degree of physical force (twisting your arm, holding you down, etc.) to make you?
Yes
No
N/A

If yes, was this person:

Male

Female

N/A

41. Have you ever had sexual intercourse (vaginal or anal intercourse between persons regardless of gender) because you were concerned about what others might think of you if you did not give in or because you thought others expected you to?
Yes
No
N/A 
Please answer the next series of questions thinking about the MOST RECENT unwanted sexual experience.

42. My most recent unwanted sexual experience was:

Unwanted sex play (fondling, kissing, or petting, but intercourse did not occur)

Unwanted attempted sexual intercourse (attempted vaginal or anal intercourse between persons regardless of sex but intercourse did not occur)

Unwanted sex acts (oral sex and/or anal or vaginal penetration by object other than the penis)

Unwanted completed sexual intercourse (vaginal or anal intercourse between persons regardless of gender)

N/A

43. How long ago did this unwanted sexual experience happen to you?

Less than 12 months ago

1-2 years ago

3-6 years ago

7-10 years ago

More than 10 years ago

N/A

44. Thinking about the time of the most recent unwanted sexual experience, what was that person's relationship to you?

Current boyfriend or someone you dated

Current girlfriend or someone you dated

Current spouse/live-in partner/fiancé(e)

Friend/acquaintance/co-worker

Parent/step-parent/parent's partner

Complete stranger

Other non-relative/neighbor

N/A 
45. How many times do you think the typical person about your age and gender has experienced:

Attempted Unwanted

Sexual Intercourse

Completed Unwanted

Sexual Intercourse

Unwanted Touching in

Sexual Manner
Never

Never

Never
$1-2$

$1-2$

$1-2$
$3-4 \quad 5+\quad$ N/A

3-4 5+ N/A

$3-4 \quad 5+\quad$ N/A

\section{Mood}

The following set of questions asks about your mood. When answering the questions in this section, please think about how many days each of the following has occurred in the past 2 weeks.

46. Over the past 2 weeks, how many days have you had little interest or pleasure in doing things?
Never
1-5 days
6-10 days
11-14 days
N/A

47. Over the past 2 weeks, how many days have you felt down, depressed or hopeless?
Never
1-5 days
6-10 days
11-14 days
N/A

48. Over the past 2 weeks, how many days have you had trouble falling or staying asleep, or sleeping too much?
Never
1-5 days
6-10 days
11-14 days
N/A

49. Over the past 2 weeks, how many days have you felt tired or had little energy?
Never
1-5 days
6-10 days
11-14 days
N/A

50. Over the past 2 weeks, how many days you had a poor appetite or eaten too much?
Never
1-5 days
6-10 days
11-14 days
N/A

51. Over the past 2 weeks, how many days have you felt bad about yourself or that you were a failure or had let yourself or your family down?
Never
1-5 days
6-10 days
11-14 days
N/A 
52. Over the past 2 weeks, how many days have you had trouble concentrating on things, such as reading or watching TV?
Never
1-5 days
6-10 days
11-14 days
N/A

53. Over the past 2 weeks, how many days have you moved or spoken so slowly that other people could notice?
Never
1-5 days
6-10 days
11-14 days
N/A

54. Over the past 2 weeks, how many days have you being so fidgety or restless that you were moving around a lot more than usual
Never
$1-5$ days
6-10 days
11-14 days
N/A

55. Has a doctor or other healthcare provider EVER told you that you had an anxiety disorder (including acute stress disorder, anxiety, generalized anxiety disorder, obsessive-compulsive disorder, panic disorder, phobia, posttraumatic stress disorder, or social anxiety disorder)?
Yes
No
Don't Know
N/A

56. Has a doctor or other healthcare provider EVER told you that you had a depressive disorder (including depression, major depression, dysthymia, or minor depression)?
Yes
No
Don't Know
N/A

57. Has your doctor or other healthcare provider told you that you have an anxiety disorder (including acute stress disorder, anxiety, generalized anxiety disorder, obsessive compulsive disorder, panic disorder, phobia, posttraumatic stress disorder, or social anxiety disorder) AFTER an unwanted sexual experience?
Yes
No
Don't Know
Did not experience an
N/A
unwanted sexual activity

58. Has a doctor or other healthcare provider told you that you have a depressive disorder (including depression, major depression, dysthymia, or minor depression) AFTER an unwanted sexual experience?
Yes
No
Don’t Know
Did not experience an 


\section{Deliberate Self Harm}

This following set of questions asks about different self-harm behaviors in which individuals sometimes engage. Please only endorse a behavior if you have done it intentionally (i.e., on purpose) without intending to die.

59. Have you ever intentionally (i.e., on purpose) performed any of the following acts (mark all that apply):

\section{Cut your skin}
Never
1-4 times
5-9 times
$10+$ times
N/A

II. Bit yourself to the extent that you broke the skin
Never
1-4 times
5-9 times
$10+$ times
N/A

III. Burned yourself (e.g., with a cigarette, lighter or match)
Never
1-4 times
5-9 times
10+ times
N/A

IV. Carved anything into your skin (e.g., words, pictures, designs or other marks)
Never
1-4 times
5-9 times
10+ times
N/A
V. Severely scratched yourself to the extent that scarring or bleeding occurred
Never
1-4 times
5-9 times
$10+$ times
N/A

VI. Banged any part of your body against something, to the extent that you caused a bruise to appear
Never
1-4 times
5-9 times
10+ times
N/A

VII. Punched yourself, to the extent that you caused a bruise to appear
Never
1-4 times
5-9 times
$10+$ times
N/A
VIII. Interfered with wound healing
Never
1-4 times
5-9 times
$10+$ times
N/A

IX. Rubbed your skin against a rough surface (e.g., sandpaper)
Never
1-4 times
5-9 times
$10+$ times
N/A

X. Rubbed glass into your skin

$\begin{array}{cccc}\text { Never } & 1-4 \text { times } & 5-9 \text { times } & \text { N/A times }\end{array}$

XI. Dripped acid onto your skin
Never
1-4 times
5-9 times
10+ times
N/A

XII. Stuck sharp objects such as needles, pins, staples, etc., into your skin, not including tattoos, ear piercings, needles for drug use or body piercing
Never
1-4 times
5-9 times
10+ times
N/A

XIII. Done anything else to hurt yourself that was not asked about in this questionnaire? If so, what 
did you do to hurt yourself?

a. Approximately how many times?

N/A

$\begin{array}{llll}\text { Never } & 1-4 \text { times } & 5-9 \text { times } & 10+\text { times }\end{array}$

60. If you have ever intentionally performed any of the acts listed in the previous question, did you do so for the AFTER an unwanted sexual experience?
Yes
No
N/A

If yes, was this your first attempt?
Yes
No
N/A

61. If you intentionally performed any of the acts listed previously AFTER or BECAUSE of an unwanted sexual experience, how long after the unwanted sexual experience did you perform the act?

Days afterward

Weeks afterward

Months afterward

Years afterward

N/A

\section{Suicide Ideation/Attempt}

The following set of questions is about suicidal thoughts and suicide attempts you have experienced in your lifetime. Suicidal thoughts are any thoughts of killing yourself, even if they were just passing and you were not seriously considering suicide. A suicide attempt is performing an action in order to try to end your own life. Please read each statement and check the appropriate response.

62. Have you ever thought about or attempted to kill yourself in your lifetime?

No

It was just a passing thought.

I briefly considered it, but not seriously.

I thought about it and was somewhat serious.

I had a plan for killing myself which I thought would work and seriously considered it.

I attempted to kill myself, but I do not think I really meant to die.

I attempted to kill myself, and I really hoped to die.

N/A 
63. How often have you thought about killing yourself in your lifetime?
Not at all
Rarely
Sometimes
Often
Very Often
N/A

64. Have you ever thought about or attempted to kill yourself in your lifetime?

No

Yes, during one short period of time

Yes, during more than one period of time

N/A

65. How likely is it that you will attempt suicide in your lifetime?

1

No Chance at all

\begin{abstract}
2
\end{abstract}
3

Some Chance
4

5

0

Great Chance

N/A

66. Would any of your problems be solved if you committed suicide?

1

No, definitely not
2

Maybe
4 Yes, definitely
0

N/A

67. If you have ever attempted suicide, did you do so AFTER an unwanted sexual experience?

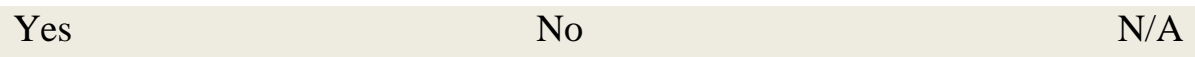

If yes, was this your first attempt?

Yes

No

N/A

68. If you attempted suicide AFTER or BECAUSE of an unwanted sexual experience, how long after the unwanted sexual experience did you attempt suicide?

Days afterward

Weeks afterward

Months afterward

Years afterward

N/A

\section{Inter-personal violence}

69. Thinking about the last $\mathbf{1 2}$ months, how often have you done any of the following things to someone that you have been on date with?

Only include it when you did it to the person first. In other words, don't count it if you 
did it in self-defense. Also, don't include if it was done in play or by accident.

I. Damaged something that belonged to them

Never $\quad 1-4$ times $\quad 5-9$ times $\quad 10+$ times $\quad$ N/A

II. Said something to hurt their feelings on purpose

Never 1-4 times $\quad 5-9$ times $\quad 10+$ times N/A

III. Threatened to hit or throw something at them

$\begin{array}{llll}\text { Never } & 1-4 \text { times } & 5-9 \text { times } & \text { N/A }\end{array}$

IV. Insulted them in front of others

\begin{tabular}{|c|c|c|c|c|c|}
\hline & Never & 1-4 times & 5-9 times & $10+$ times & N/A \\
\hline V. & \multicolumn{5}{|c|}{ Scratched them } \\
\hline & Never & $1-4$ times & 5-9 times & $10+$ times & N/A \\
\hline
\end{tabular}

VI. Put down their looks

$\begin{array}{llllr} & \text { Never } 1-4 \text { times } & 5-9 \text { times } & 10+\text { times } & \text { N/A } \\ \text { VII. } & \text { Hit or slapped them } & & \\ & \text { Never } \quad 1-4 \text { times } & 5-9 \text { times } & 10+\text { times } & \text { N/A }\end{array}$

VIII. Would not let them do things with other people

Never 1-4 times 5-9 times $\quad 10+$ times N/A

IX. Made them describe where they were every minute of the day

Never $\quad 1-4$ times $\quad 5-9$ times $\quad 10+$ times N/A

X. Slammed or held them against a wall

$\begin{array}{llll}\text { Never } \quad 1-4 \text { times } & 5-9 \text { times } & \text { N/A }\end{array}$

XI. Did something just to make them jealous

\begin{tabular}{|c|c|c|c|c|c|}
\hline & Never & 1-4 times & 5-9 times & $10+$ times & N/A \\
\hline XII. & Kicked them & & & & \\
\hline & Never & 1-4 times & 5-9 times & $10+$ times & N/A \\
\hline
\end{tabular}

XIII. Pushed, grabbed or shoved them

\begin{tabular}{|llccc} 
XIV. & $\begin{array}{l}\text { Never } \\
\text { Forced them to engage in sex play (fondling, kissing, or petting, but not } \\
\text { intercourse) }\end{array}$ & N/A \\
\hline XV. & $\begin{array}{l}\text { Never } \\
\begin{array}{l}\text { Forced them to engage in sex acts (anal or oral intercourse or penetration by } \\
\text { object other than the penis) }\end{array}\end{array}$ & N/A \\
\hline
\end{tabular}



Never
1-4 times
5-9 times
$10+$ times
N/A

XVI. Attempted sexual intercourse but intercourse did not occur
Never
1-4 times
5-9 times
$10+$ times
N/A

XVII. Forced them to have sexual intercourse with you

Never 1-4 times $\quad 5-9$ times $\quad 10+$ times N/A

XVIII. Threw something at them with something that could hurt.
Never
1-4 times
5-9 times
$10+$ times
N/A

XIX. Punched or hit them with something that could hurt
Never
1-4 times
5-9 times
$10+$ times
N/A

XX. Threatened or injured them with a knife or gun

Never 1-4 times $\quad 5-9$ times $10+$ times N/A

XXI. Hurt them badly enough to need bandages or care from a doctor or nurse
Never
1-4 times
5-9 times
$10+$ times
N/A

70. In the past 12 months, how often have you done any of the following things to someone besides a date? Again, think of the things that you have done to people about your age. These can be strangers, friends or other people you know. Don't include things done to family members or sibling.

Only include it when you did it to the person first. In other words, don't count it if you did it in self-defense. Also, don't include if it was done in play or by accident.

I. Damaged something that belonged to them

Never 1-4 times 5-9 times 10+ times N/A

II. Said something to hurt their feelings on purpose

Never 1-4 times $\quad 5-9$ times $10+$ times N/A

III. Threatened to hit or throw something at them

\begin{tabular}{|c|c|c|c|c|c|}
\hline \multirow[b]{2}{*}{ IV. } & Never & 1-4 times & 5-9 times & $10+$ times & N/A \\
\hline & \multicolumn{5}{|c|}{ Insulted them in front of others } \\
\hline & Never & $1-4$ times & 5-9 times & $10+$ times & N/A \\
\hline \multirow[t]{2}{*}{ V. } & \multicolumn{5}{|c|}{ Scratched them } \\
\hline & Never & 1-4 times & $5-9$ times & $10+$ times & N/A \\
\hline \multirow[t]{2}{*}{ VI. } & \multicolumn{5}{|c|}{ Put down their looks } \\
\hline & Never & 1-4 times & 5-9 times & $10+$ times & N/A \\
\hline
\end{tabular}

VII. Hit or slapped them

Never $\quad 1-4$ times $\quad 5-9$ times $\quad 10+$ times $\quad$ N/A


VIII. Slammed or held them against a wall

$$
\text { Never 1-4 times } \quad 5-9 \text { times } \quad 10+\text { times N/A }
$$

IX. Did something just to make them jealous

\begin{tabular}{|c|c|c|c|c|c|}
\hline & Never & 1-4 times & 5-9 times & $10+$ times & N/A \\
\hline X. & Kicked them & & & & \\
\hline & Never & 1-4 times & 5-9 times & $10+$ times & N/A \\
\hline
\end{tabular}

XI. Pushed, grabbed or shoved them

Never 1-4 times 5-9 times 10+ times N/A

XII. Forced them to engage in sex play (fondling, kissing, or petting, but not intercourse)

Never 1-4 times 5-9 times 10+ times N/A

XIII. Forced them to engage in sex acts (anal or oral intercourse or penetration by object other than the penis)
Never
1-4 times
5-9 times
$10+$ times
N/A

XIV. Attempted sexual intercourse but intercourse did not occur
Never
1-4 times
5-9 times
$10+$ times
N/A

XV. Forced them to have sex with you
Never
1-4 times
5-9 times
$10+$ times
N/A

XVI. Threw something at them with something that could hurt.
Never
1-4 times
5-9 times
$10+$ times
N/A

XVII. Punched or hit them with something that could hurt
Never
1-4 times
5-9 times
$10+$ times
N/A

XVIII. Threatened or injured them with a knife or gun.
Never
1-4 times
5-9 times
$10+$ times
N/A

XIX. Hurt them badly enough to need bandages or care from a doctor or nurse

Never $\quad 1-4$ times $\quad 5-9$ times $\quad 10+$ times $\quad$ N/A




\section{Demographics}

71. What is your sex?
Male
Female

72. What is your age
$18-19$ yrs
20-21yrs
22-23yrs
24-25yrs
$25+$ yrs

73. Year in school:

$1^{\text {st }}$ year undergraduate

$2^{\text {nd }}$ year undergraduate

$3^{\text {rd }}$ year undergraduate

$4^{\text {th }}$ year undergraduate

$5^{\text {th }}$ year or more undergraduate

Graduate or Professional

Other

74. How do you describe yourself?

White - not Hispanic

Black - not Hispanic

Hispanic or Latino

Asian or Pacific Islander

American Indian or Alaskan Native

Other

75. What is your current relationship status?

Single

Separated

Married/cohabitating

Divorced 
Engaged or committed dating

Widowed

76. Which of the following describes you the best?

Heterosexual

Bisexual

Gay/Lesbian

Unsure

77. Are you a member of a social fraternity or sorority?

Yes No

78. How would you define an "unwanted sexual experience?"

79. Please provide any comments or suggestions you have regarding the survey.

This is the end of the survey.

Thank you! 
Appendix B 


\section{Appendix B}

Definition of Types of Sexual Victimization:

Sexual Contact Have you ever experienced sex play (fondling, kissing, or petting but no intercourse) when you didn't want to because you were overwhelmed by the person/partner's continual argument and pressure?

Have you ever experienced sex play (fondling, kissing, or petting but no intercourse) when you didn't want to because the person/partner used his/her position of authority (boss, teacher, camp counselor, supervisor, family member etc.) to make you?

Have you ever experienced sex play (fondling, kissing, or petting but no intercourse)when you didn't want to because the person/partner threatened or used some degree of physical force (twisting your arm, holding you down, etc.) to make you?

Coercion

Have you ever experienced sex acts (oral and/or anal or vaginal penetration by an object other than penis) when you didn't want to because you were overwhelmed by the person/partner's continual argument and pressure?

Have you ever experienced sex acts (oral and/or anal or vaginal penetration by an object other than penis) when you didn't want to because the person/partner used his/her position of authority (boss, teacher, camp counselor, supervisor, family member etc.) to make you?

Have you ever had sexual intercourse (vaginal or anal intercourse between person regardless of gender) when you didn't want to because you were overwhelmed by the person/partner's continual argument and pressure?

Have you ever had sexual intercourse (vaginal or anal intercourse between person regardless of gender) when you didn't want to because the person/partner used his/her position of authority (boss, teacher, camp counselor, supervisor, family member etc.) to make you?

Attempted Rape Have you ever had a person/partner attempted sexual intercourse (attempted vaginal or anal intercourse between persons regardless of gender) when you didn't want to because you were overwhelmed by the person/partner's continual argument and pressure?

Have you ever had a person/partner attempted sexual intercourse (attempted vaginal or anal intercourse between persons regardless of gender) when you didn't want to because the person/partner used his/her position of authority (boss, teacher, camp counselor, supervisor, family member etc.) to make you? 
Have you ever had a person/partner attempted sexual intercourse (attempted vaginal or anal intercourse between persons regardless of gender) when you didn't want to because the person/partner gave you alcohol or drugs?

Have you ever had a person/partner attempted sexual intercourse (attempted vaginal or anal intercourse between persons regardless of gender) when you didn't want to because the person/partner threatened or used some degree of physical force (twisting your arm, holding you down, etc.) to make you?

Completed Rape

Sexual Assault with an object

Have you ever had sexual intercourse (vaginal or anal intercourse between person regardless of gender) when you didn't want to because the person/partner gave you alcohol or drugs?

Have you ever had sexual intercourse (vaginal or anal intercourse between person regardless of gender) when you didn't want to because you consumed too much alcohol or drugs willingly?

Have you ever had sexual intercourse (vaginal or anal intercourse between person regardless of gender) when you didn't want to because the person/partner threatened or used some degree of physical force (twisting your arm, holding you down, etc.) to make you?

Have you ever experienced sex acts (oral and/or anal or vaginal penetration by an object other than penis) when you didn't want to because the person/partner gave you alcohol or drugs?

Have you ever experienced sex acts (oral and/or anal or vaginal penetration by an object other than penis) when you didn't want to because you consumed too much alcohol or drugs willingly?

Have you ever experienced sex acts (oral and/or anal or vaginal penetration by an object other than penis)when you didn't want to because the person/partner threatened or used some degree of physical force (twisting your arm, holding you down, etc.) to make you?

Drug or Alcohol

Given by Perpetrator
Have you ever experienced sex play (fondling, kissing, or petting but no intercourse) when you didn't want to because the person/partner gave you alcohol or drugs?

Have you ever had a person/partner attempted sexual intercourse (attempted vaginal or anal intercourse between persons regardless of gender) when you didn't want to because the person/partner gave you alcohol or drugs?

Have you ever experienced sex acts (oral and/or anal or vaginal penetration by an object other than penis) when you didn't want to 
because the person/partner gave you alcohol or drugs?

Have you ever had sexual intercourse (vaginal or anal intercourse between person regardless of gender) when you didn't want to because the person/partner gave you alcohol or drugs?

Drug or Alcohol Use by yourself
Have you ever experienced sex play (fondling, kissing, or petting but no intercourse) when you didn't want to because you consumed too much alcohol or drugs willingly?

Have you had a person/partner attempted sexual intercourse (attempted vaginal or anal intercourse between persons regardless of gender) when you didn't want to because you consumed too much alcohol or drugs willingly?

Have you ever experienced sex acts (oral and/or anal or vaginal penetration by an object other than penis) when you didn't want to because you consumed too much alcohol or drugs willingly?

Have you had sexual intercourse (vaginal or anal intercourse between person regardless of gender) when you didn't want to because you consumed too much alcohol or drugs willingly?

\section{External Pressure}

Have you ever experienced sex play (fondling, kissing, or petting but no intercourse) because you were concerned about what others might think of you if you did not give in or because you thought others expected you to?

Have you ever had a person/partner attempted sexual intercourse (attempted vaginal or anal intercourse between persons regardless of gender) because you were concerned about what others might think of you if you did not give in or because you thought others expected you to?

Have you ever experienced sex acts (oral and/or anal or vaginal penetration by an object other than penis) because you were concerned about what others might think of you if you did not give in or because you thought others expected you to?

Have you ever had sexual intercourse (vaginal or anal intercourse between persons regardless of gender) because you were concerned about what others might think of you if you did not give in or because you thought others expected you to? 


\section{Appendix C}




\section{Appendix C}

\section{Data Quality and Power Analysis}

Total sample size: 780

- $\quad$ Males $=222$

- Females $=554$

Time to take the survey:

\begin{tabular}{|l|c|c|c|c|c|}
\hline & $\mathbf{N}$ & $\begin{array}{c}\text { Mean Time } \\
(\mathbf{m i n})\end{array}$ & Std. Dev & Minimum & Maximum \\
\hline All & 780 & 18.72 & 8.01 & 5.00 & 66.00 \\
\hline Males & 212 & 18.42 & 8.51 & 6.00 & 66.00 \\
\hline Females & 537 & 18.92 & 7.77 & 5.00 & 61.00 \\
\hline
\end{tabular}

\section{Data quality measures:}

- Time to take the survey

- Sexual violence questions

- Demographic questions

- Outcome measures

Table 1: Cleaning of Data

\begin{tabular}{|l|l|l|l|}
\hline & All & Male & Female \\
\hline Total Sample size & 780 & 222 & 554 \\
\hline Only if duration to take the survey was 10 min or more & 735 & 197 & 538 \\
\hline All cases if person answered Yes or No to SV questions & 725 & 195 & 530 \\
\hline Data with completed demographics (Final sample size) & 720 & 194 & 526 \\
\hline
\end{tabular}

Sexual Victimization: Similar measures were used by other studies ${ }^{148,177,178}$ in college population and can be used for direct comparisons. Also these categories are somewhat similar to the legal definitions of sexual assault (see appendix B for details). 


\section{Power Analysis:}

- Hypothesis 2.1: Victimized males are more likely to perpetrate intimate partner violence when compared with non-victimized males.

Comparison Groups: Victimized males vs. non-victimized males

Outcome Measure: intimate partner violence (physical, emotional, sexual)

Table 1A: Sexual Contact

\begin{tabular}{|c|c|c|c|c|c|c|c|c|}
\hline $\begin{array}{l}\text { Aggressive } \\
\text { Behavior }\end{array}$ & $\begin{array}{l}\text { Current } \\
\text { Sample } \\
\text { size }\end{array}$ & $\begin{array}{l}\text { Victimized } \\
\text { Males } \\
\% \text { (n/Total } \\
\text { Victims) }\end{array}$ & $\begin{array}{c}\text { Not } \\
\text { Victimized } \\
\text { Males } \\
\% \text { (n/Total } \\
\text { Non-victims) }\end{array}$ & $\%$ Difference & $\begin{array}{c}\text { Power } \\
\text { With } \\
\text { Sample } \\
\text { current } \\
\text { size } \\
(\%) \\
\end{array}$ & $\begin{array}{c}\text { Power } \\
\text { Sample } \\
\text { size = 300 } \\
(\%)\end{array}$ & $\begin{array}{c}\text { Power } \\
\text { Sample } \\
\text { size }=500 \\
(\%)\end{array}$ & $\begin{array}{c}\text { Power } \\
\text { Sample } \\
\text { size }= \\
1000 \\
(\%)\end{array}$ \\
\hline $\begin{array}{l}\text { Perpetrated intimate- } \\
\text { partner physical } \\
\text { violence }\end{array}$ & 157 & $28.21(11 / 38)$ & $6.78(8 / 118)$ & 21.43 & 100 & 100 & 100 & 100 \\
\hline $\begin{array}{l}\text { Perpetrated intimate- } \\
\text { partner emotional } \\
\text { violence }\end{array}$ & 157 & $72.50(29 / 40)$ & $59.83(70 / 117)$ & 12.67 & 100 & 100 & 100 & 100 \\
\hline $\begin{array}{l}\text { Perpetrated intimate- } \\
\text { partner sexual } \\
\text { violence }\end{array}$ & 158 & $29.27(12 / 41)$ & $11.11(13 / 117)$ & 18.16 & 96 & 100 & 100 & 100 \\
\hline
\end{tabular}

Table 1B: Coercion

\begin{tabular}{|c|c|c|c|c|c|c|c|c|}
\hline $\begin{array}{l}\text { Aggressive } \\
\text { Behavior }\end{array}$ & $\begin{array}{c}\text { Current } \\
\text { Sample } \\
\text { size }\end{array}$ & $\begin{array}{c}\text { Victimized } \\
\text { Males } \\
\text { \% (n/Total } \\
\text { Victims) }\end{array}$ & $\begin{array}{c}\text { Not } \\
\text { Victimized } \\
\text { Males } \\
\% \text { (n/Total } \\
\text { Non-victims) }\end{array}$ & \% Difference & $\begin{array}{c}\text { Power } \\
\text { With } \\
\text { Sample } \\
\text { current } \\
\text { size } \\
(\%)\end{array}$ & $\begin{array}{c}\text { Power } \\
\text { Sample } \\
\text { size }=\mathbf{3 0 0} \\
(\%)\end{array}$ & $\begin{array}{c}\text { Power } \\
\text { Sample } \\
\text { size }=500 \\
(\%)\end{array}$ & $\begin{array}{c}\text { Power } \\
\text { Sample } \\
\text { size }= \\
1000 \\
(\%)\end{array}$ \\
\hline $\begin{array}{l}\text { Perpetrated intimate- } \\
\text { partner physical } \\
\text { violence }\end{array}$ & 142 & $42.86(9 / 21)$ & $6.61(8 / 121)$ & 36.25 & 100 & 100 & 100 & 100 \\
\hline $\begin{array}{l}\text { Perpetrated intimate- } \\
\text { partner emotional } \\
\text { violence }\end{array}$ & 141 & $80.95(17 / 21)$ & $59.17(71 / 120)$ & 21.78 & 100 & 100 & 100 & 100 \\
\hline $\begin{array}{l}\text { Perpetrated intimate- } \\
\text { partner sexual } \\
\text { violence }\end{array}$ & 141 & $28.57(21)$ & $12.50(15 / 120)$ & 16.07 & 99.9 & 100 & 100 & 100 \\
\hline
\end{tabular}

Table 1C: Attempted Rape

\begin{tabular}{|c|c|c|c|c|c|c|c|c|}
\hline $\begin{array}{l}\text { Aggressive } \\
\text { Behavior }\end{array}$ & $\begin{array}{c}\text { Current } \\
\text { Sample } \\
\text { size }\end{array}$ & $\begin{array}{c}\text { Victimized } \\
\text { Males } \\
\text { \% (n/Total } \\
\text { Victims) }\end{array}$ & $\begin{array}{c}\text { Not } \\
\text { Victimized } \\
\text { Males } \\
\% \text { (n/Total } \\
\text { Non-victims) }\end{array}$ & \% Difference & $\begin{array}{c}\text { Power } \\
\text { With } \\
\text { Sample } \\
\text { current } \\
\text { size } \\
(\%)\end{array}$ & $\begin{array}{c}\text { Power } \\
\text { Sample } \\
\text { size }=300 \\
(\%)\end{array}$ & $\begin{array}{c}\text { Power } \\
\text { Sample } \\
\text { size }=500 \\
(\%)\end{array}$ & $\begin{array}{c}\text { Power } \\
\text { Sample } \\
\text { size = } \\
1000 \\
(\%)\end{array}$ \\
\hline $\begin{array}{l}\text { Perpetrated intimate- } \\
\text { partner physical } \\
\text { violence }\end{array}$ & 151 & $34.78(8 / 23)$ & $5.47(7 / 128)$ & 29.31 & 100 & 100 & 100 & 100 \\
\hline $\begin{array}{l}\text { Perpetrated intimate- } \\
\text { partner emotional } \\
\text { violence }\end{array}$ & 151 & $82.61(19 / 23)$ & $59.38(76 / 128)$ & 23.23 & 100 & 100 & 100 & 100 \\
\hline $\begin{array}{l}\text { Perpetrated intimate- } \\
\text { partner sexual } \\
\text { violence }\end{array}$ & 151 & $30.43(7 / 23)$ & $12.50(16 / 128)$ & 17.93 & 100 & 100 & 100 & 100 \\
\hline
\end{tabular}


Table 1D: Penetration with an object

\begin{tabular}{|c|c|c|c|c|c|c|c|c|}
\hline $\begin{array}{l}\text { Aggressive } \\
\text { Behavior }\end{array}$ & $\begin{array}{c}\text { Current } \\
\text { Sample } \\
\text { size }\end{array}$ & $\begin{array}{l}\text { Victimized } \\
\text { Males } \\
\% \text { (n/Total } \\
\text { Victims) }\end{array}$ & $\begin{array}{c}\text { Not } \\
\text { Victimized } \\
\text { Males } \\
\% \text { (n/Total } \\
\text { Non-victims) }\end{array}$ & \% Difference & $\begin{array}{c}\text { Power } \\
\text { With } \\
\text { Sample } \\
\text { current } \\
\text { size } \\
(\%)\end{array}$ & $\begin{array}{c}\text { Power } \\
\text { Sample } \\
\text { size }=\mathbf{3 0 0} \\
(\%)\end{array}$ & $\begin{array}{c}\text { Power } \\
\text { Sample } \\
\text { size = 500 } \\
(\%)\end{array}$ & $\begin{array}{c}\text { Power } \\
\text { Sample } \\
\text { size = } \\
1000 \\
(\%)\end{array}$ \\
\hline $\begin{array}{l}\text { Perpetrated intimate- } \\
\text { partner physical } \\
\text { violence }\end{array}$ & 160 & $19.51(8 / 41)$ & $9.24(11 / 119)$ & 10.27 & 98.1 & 100 & 100 & 100 \\
\hline $\begin{array}{l}\text { Perpetrated intimate- } \\
\text { partner emotional } \\
\text { violence }\end{array}$ & 160 & $80.49(33 / 41)$ & $57.14(68 / 119)$ & 23.35 & 100 & 100 & 100 & 100 \\
\hline $\begin{array}{l}\text { Perpetrated intimate- } \\
\text { partner sexual } \\
\text { violence }\end{array}$ & 161 & $26.83(11 / 41)$ & $12.50(15 / 120)$ & 14.33 & 99.8 & 100 & 100 & 100 \\
\hline
\end{tabular}

Table 1E: Sexual Intercourse

\begin{tabular}{|c|c|c|c|c|c|c|c|c|}
\hline $\begin{array}{l}\text { Aggressive } \\
\text { Behavior }\end{array}$ & $\begin{array}{c}\text { Current } \\
\text { Sample } \\
\text { size }\end{array}$ & $\begin{array}{l}\text { Victimized } \\
\text { Males } \\
\% \text { (n/Total } \\
\text { Victims) }\end{array}$ & $\begin{array}{c}\text { Not } \\
\text { Victimized } \\
\text { Males } \\
\% \text { (n/Total } \\
\text { Non-victims) }\end{array}$ & \% Difference & $\begin{array}{c}\text { Power } \\
\text { With } \\
\text { Sample } \\
\text { current } \\
\text { size } \\
(\%)\end{array}$ & $\begin{array}{c}\text { Power } \\
\text { Sample } \\
\text { size }=\mathbf{3 0 0} \\
(\%)\end{array}$ & $\begin{array}{c}\text { Power } \\
\text { Sample } \\
\text { size = 500 } \\
(\%)\end{array}$ & $\begin{array}{c}\text { Power } \\
\text { Sample } \\
\text { size = } \\
1000 \\
(\%)\end{array}$ \\
\hline $\begin{array}{l}\text { Perpetrated intimate- } \\
\text { partner physical } \\
\text { violence }\end{array}$ & 146 & $15.22(7 / 46)$ & $9.00(9 / 100)$ & 6.22 & 78.3 & 95.4 & 99.5 & 100 \\
\hline $\begin{array}{l}\text { Perpetrated intimate- } \\
\text { partner emotional } \\
\text { violence }\end{array}$ & 146 & $80.85(38 / 47)$ & $54.55(54 / 99)$ & 26.3 & 100 & 100 & 100 & 100 \\
\hline $\begin{array}{l}\text { Perpetrated intimate- } \\
\text { partner sexual } \\
\text { violence }\end{array}$ & 146 & $19.15(9 / 47)$ & $12.12(12 / 99)$ & 7.03 & 73.2 & 96 & 100 & 100 \\
\hline
\end{tabular}

\section{- Hypothesis 2.2: Victimized males are more likely to perpetrate non-partner interpersonal violence when compared with non-victimized males.}

Comparison Groups: Victimized males vs. non-victimized males

Outcome Measure: Non-partner interpersonal violence (physical, emotional, sexual)

Table 2A: Sexual Contact

\begin{tabular}{|c|c|c|c|c|c|c|c|c|}
\hline $\begin{array}{l}\text { Aggressive } \\
\text { Behavior }\end{array}$ & $\begin{array}{c}\text { Current } \\
\text { Sample } \\
\text { size }\end{array}$ & $\begin{array}{l}\text { Victimized } \\
\text { Males } \\
\% \text { (n/Total } \\
\text { Victims) }\end{array}$ & $\begin{array}{c}\text { Not } \\
\text { Victimized } \\
\text { Males } \\
\% \text { (n/Total } \\
\text { Non-victims) }\end{array}$ & \% Difference & $\begin{array}{c}\text { Power } \\
\text { With } \\
\text { Sample } \\
\text { current } \\
\text { size } \\
(\%)\end{array}$ & $\begin{array}{c}\text { Power } \\
\text { Sample } \\
\text { size }=\mathbf{3 0 0} \\
(\%)\end{array}$ & $\begin{array}{c}\text { Power } \\
\text { Sample } \\
\text { size = 500 } \\
(\%)\end{array}$ & $\begin{array}{c}\text { Power } \\
\text { Sample } \\
\text { size = } \\
1000 \\
(\%)\end{array}$ \\
\hline $\begin{array}{l}\text { Perpetrated } \\
\text { interpersonal } \\
\text { physical violence }\end{array}$ & 160 & $58.54(24 / 41)$ & $52.94(63 / 119)$ & 5.6 & 41 & 61.9 & 80.9 & 100 \\
\hline $\begin{array}{l}\text { Perpetrated } \\
\text { interpersonal } \\
\text { emotional violence }\end{array}$ & 160 & $73.17(30 / 41)$ & $62.18(74 / 119)$ & 10.99 & 90.9 & 100 & 100 & 100 \\
\hline $\begin{array}{l}\text { Perpetrated } \\
\text { interpersonal sexual } \\
\text { violence }\end{array}$ & 159 & $12.50(5 / 40)$ & $9.24(11 / 119)$ & 3.26 & 42.2 & 60.5 & 77.8 & 95.3 \\
\hline
\end{tabular}


Table 2B: Coercion

\begin{tabular}{|c|c|c|c|c|c|c|c|c|}
\hline $\begin{array}{l}\text { Aggressive } \\
\text { Behavior }\end{array}$ & $\begin{array}{l}\text { Current } \\
\text { Sample } \\
\text { size }\end{array}$ & $\begin{array}{l}\text { Victimized } \\
\text { Males } \\
\% \text { (n/Total } \\
\text { Victims) }\end{array}$ & $\begin{array}{c}\text { Not } \\
\text { Victimized } \\
\text { Males } \\
\% \text { (n/Total } \\
\text { Non-victims) }\end{array}$ & \% Difference & $\begin{array}{c}\text { Power } \\
\text { With } \\
\text { Sample } \\
\text { current } \\
\text { size } \\
(\%)\end{array}$ & $\begin{array}{c}\text { Power } \\
\text { Sample } \\
\text { size }=\mathbf{3 0 0} \\
(\%)\end{array}$ & $\begin{array}{c}\text { Power } \\
\text { Sample } \\
\text { size }=500 \\
(\%)\end{array}$ & $\begin{array}{c}\text { Power } \\
\text { Sample } \\
\text { size }= \\
1000 \\
(\%)\end{array}$ \\
\hline $\begin{array}{l}\text { Perpetrated } \\
\text { interpersonal } \\
\text { physical violence }\end{array}$ & 143 & $71.43(15 / 21)$ & $51.64(63 / 122)$ & 19.79 & 100 & 100 & 100 & 100 \\
\hline $\begin{array}{l}\text { Perpetrated } \\
\text { interpersonal } \\
\text { emotional violence }\end{array}$ & 143 & $85.71(18 / 21)$ & $63.93(78 / 122)$ & 21.78 & 100 & 100 & 100 & 100 \\
\hline $\begin{array}{l}\text { Perpetrated } \\
\text { interpersonal sexual } \\
\text { violence }\end{array}$ & 143 & $23.81(5 / 21)$ & $8.20(10 / 122)$ & 15.61 & 100 & 100 & 100 & 100 \\
\hline
\end{tabular}

Table 2C: Attempted Sexual Intercourse

\begin{tabular}{|c|c|c|c|c|c|c|c|c|}
\hline $\begin{array}{l}\text { Aggressive } \\
\text { Behavior }\end{array}$ & $\begin{array}{c}\text { Current } \\
\text { Sample } \\
\text { size }\end{array}$ & 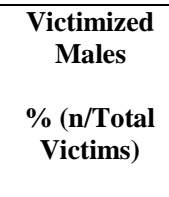 & $\begin{array}{c}\text { Not } \\
\text { Victimized } \\
\text { Males } \\
\% \text { (n/Total } \\
\text { Non-victims) }\end{array}$ & \% Difference & $\begin{array}{c}\text { Power } \\
\text { With } \\
\text { Sample } \\
\text { current } \\
\text { size } \\
(\%)\end{array}$ & $\begin{array}{c}\text { Power } \\
\text { Sample } \\
\text { size = 300 } \\
(\%)\end{array}$ & $\begin{array}{c}\text { Power } \\
\text { Sample } \\
\text { size = 500 } \\
(\%)\end{array}$ & $\begin{array}{c}\text { Power } \\
\text { Sample } \\
\text { size }= \\
1000 \\
(\%)\end{array}$ \\
\hline $\begin{array}{l}\text { Perpetrated } \\
\text { interpersonal } \\
\text { physical violence }\end{array}$ & 153 & $69.57(16 / 23)$ & $51.54(67 / 130)$ & 18.03 & 99.9 & 100 & 100 & 100 \\
\hline $\begin{array}{l}\text { Perpetrated } \\
\text { interpersonal } \\
\text { emotional violence }\end{array}$ & 153 & $78.26(18 / 23)$ & $62.31(81 / 130)$ & 15.95 & 99.8 & 100 & 100 & 100 \\
\hline $\begin{array}{l}\text { Perpetrated } \\
\text { interpersonal sexual } \\
\text { violence }\end{array}$ & 153 & $21.74(5 / 23)$ & $6.92(9 / 130)$ & 14.82 & 100 & 100 & 100 & 100 \\
\hline
\end{tabular}

Table 2D: Penetration with object

\begin{tabular}{|c|c|c|c|c|c|c|c|c|}
\hline $\begin{array}{l}\text { Aggressive } \\
\text { Behavior }\end{array}$ & $\begin{array}{l}\text { Current } \\
\text { Sample } \\
\text { size }\end{array}$ & $\begin{array}{l}\text { Victimized } \\
\text { Males } \\
\text { \% (n/Total } \\
\text { Victims) }\end{array}$ & $\begin{array}{c}\text { Not } \\
\text { Victimized } \\
\text { Males } \\
\% \text { (n/Total } \\
\text { Non-victims) }\end{array}$ & \% Difference & $\begin{array}{c}\text { Power } \\
\text { With } \\
\text { Sample } \\
\text { current } \\
\text { size } \\
(\%)\end{array}$ & $\begin{array}{c}\text { Power } \\
\text { Sample } \\
\text { size = 300 } \\
(\%)\end{array}$ & $\begin{array}{c}\text { Power } \\
\text { Sample } \\
\text { size }=500 \\
(\%)\end{array}$ & $\begin{array}{c}\text { Power } \\
\text { Sample } \\
\text { size }= \\
1000 \\
(\%)\end{array}$ \\
\hline $\begin{array}{l}\text { Perpetrated } \\
\text { interpersonal } \\
\text { physical violence }\end{array}$ & 163 & $68.29(28 / 41)$ & $47.54(58 / 122)$ & 20.75 & 100 & 100 & 100 & 100 \\
\hline $\begin{array}{l}\text { Perpetrated } \\
\text { interpersonal } \\
\text { emotional violence }\end{array}$ & 163 & $78.05(32 / 41)$ & $58.20(71 / 122)$ & 19.85 & 100 & 100 & 100 & 100 \\
\hline $\begin{array}{l}\text { Perpetrated } \\
\text { interpersonal sexual } \\
\text { violence }\end{array}$ & 162 & $19.51(8 / 41)$ & $6.61(8 / 121)$ & 12.9 & 99.9 & 100 & 100 & 100 \\
\hline
\end{tabular}


Table 2E: Sexual Intercourse

\begin{tabular}{|c|c|c|c|c|c|c|c|c|}
\hline $\begin{array}{l}\text { Aggressive } \\
\text { Behavior }\end{array}$ & $\begin{array}{l}\text { Current } \\
\text { Sample } \\
\text { size }\end{array}$ & $\begin{array}{l}\text { Victimized } \\
\text { Males } \\
\% \text { (n/Total } \\
\text { Victims) }\end{array}$ & $\begin{array}{c}\text { Not } \\
\text { Victimized } \\
\text { Males } \\
\% \text { (n/Total } \\
\text { Non-victims) }\end{array}$ & \% Difference & $\begin{array}{c}\text { Power } \\
\text { With } \\
\text { Sample } \\
\text { current } \\
\text { size } \\
(\%) \\
\end{array}$ & $\begin{array}{c}\text { Power } \\
\text { Sample } \\
\text { size = 300 } \\
(\%)\end{array}$ & $\begin{array}{c}\text { Power } \\
\text { Sample } \\
\text { size }=500 \\
(\%)\end{array}$ & $\begin{array}{c}\text { Power } \\
\text { Sample } \\
\text { size }= \\
1000 \\
(\%)\end{array}$ \\
\hline $\begin{array}{l}\text { Perpetrated } \\
\text { interpersonal } \\
\text { physical violence }\end{array}$ & 148 & $70.21(33 / 47)$ & $47.52(48 / 101)$ & 22.69 & 100 & 100 & 100 & 100 \\
\hline $\begin{array}{l}\text { Perpetrated } \\
\text { interpersonal } \\
\text { emotional violence }\end{array}$ & 148 & $74.47(35 / 47)$ & $60.40(61 / 101)$ & 14.07 & 98.1 & 100 & 100 & 100 \\
\hline $\begin{array}{l}\text { Perpetrated } \\
\text { interpersonal sexual } \\
\text { violence }\end{array}$ & 148 & $17.02(8 / 47)$ & $5.94(6 / 101)$ & 11.08 & 99.5 & 100 & 100 & 100 \\
\hline
\end{tabular}

\section{Hypothesis 2.3: Victimized males are at lower risk of deliberate self-harm when compared with victimized females.}

Comparison Groups: Victimized males vs. victimized females

Outcome Measure: deliberate self-harm

Table 3A: Sexual Contact

\begin{tabular}{|c|c|c|c|c|c|c|c|c|}
\hline Aggressive Behavior & $\begin{array}{c}\text { Current } \\
\text { Sample } \\
\text { size }\end{array}$ & $\begin{array}{c}\text { Victimized } \\
\text { Males } \\
\% \text { (n/Total } \\
\text { male Victims) }\end{array}$ & $\begin{array}{l}\text { Victimized } \\
\text { Females } \\
\% \text { (n/Total } \\
\text { female } \\
\text { victims) }\end{array}$ & $\begin{array}{c}\% \\
\text { Difference }\end{array}$ & $\begin{array}{l}\text { Power } \\
\text { With } \\
\text { Sample } \\
\text { current } \\
\text { size } \\
(\%)\end{array}$ & $\begin{array}{c}\text { Power } \\
\text { Sample } \\
\text { size }= \\
300 \\
(\%)\end{array}$ & $\begin{array}{c}\text { Power } \\
\text { Sample } \\
\text { size }= \\
\mathbf{5 0 0} \\
(\%)\end{array}$ & $\begin{array}{c}\text { Power } \\
\text { Sample } \\
\text { size }= \\
1000 \\
(\%)\end{array}$ \\
\hline Deliberate Self Harm & 279 & $\begin{array}{c}57.50 \\
(23 / 40)\end{array}$ & $\begin{array}{c}56.07 \\
(134 / 239)\end{array}$ & 1.43 & 12.1 & 12.5 & 15.8 & 23.1 \\
\hline
\end{tabular}

Table 3B: Coercion

\begin{tabular}{|c|c|c|c|c|c|c|c|c|}
\hline $\begin{array}{l}\text { Aggressive } \\
\text { Behavior }\end{array}$ & $\begin{array}{c}\text { Current } \\
\text { Sample } \\
\text { size }\end{array}$ & $\begin{array}{c}\text { Victimized } \\
\text { Males } \\
\% \text { (n/Total } \\
\text { male Victims) }\end{array}$ & $\begin{array}{l}\text { Victimized } \\
\text { Females } \\
\% \text { (n/Total } \\
\text { female } \\
\text { victims) }\end{array}$ & \% Difference & $\begin{array}{l}\text { Power } \\
\text { With } \\
\text { Sample } \\
\text { current } \\
\text { size } \\
(\%)\end{array}$ & $\begin{array}{c}\text { Power } \\
\text { Sample } \\
\text { size = 300 } \\
(\%)\end{array}$ & $\begin{array}{c}\text { Power } \\
\text { Sample } \\
\text { size }=500 \\
(\%)\end{array}$ & $\begin{array}{c}\text { Power } \\
\text { Sample } \\
\text { size }= \\
1000 \\
(\%)\end{array}$ \\
\hline $\begin{array}{l}\text { Deliberate Self } \\
\text { Harm }\end{array}$ & 182 & $\begin{array}{c}80.95 \\
(17 / 21)\end{array}$ & $\begin{array}{c}59.01 \\
(95 / 161)\end{array}$ & 21.94 & 100 & 100 & 100 & 100 \\
\hline
\end{tabular}

Table 3C: Attempted Sexual Intercourse

\begin{tabular}{|c|c|c|c|c|c|c|c|c|}
\hline $\begin{array}{l}\text { Aggressive } \\
\text { Behavior }\end{array}$ & $\begin{array}{c}\text { Current } \\
\text { Sample } \\
\text { size }\end{array}$ & $\begin{array}{c}\text { Victimized } \\
\text { Males } \\
\text { \% (n/Total } \\
\text { male Victims) }\end{array}$ & $\begin{array}{c}\text { Victimized } \\
\text { Females } \\
\% \text { (n/Total } \\
\text { female } \\
\text { victims) }\end{array}$ & \% Difference & $\begin{array}{c}\text { Power } \\
\text { With } \\
\text { Sample } \\
\text { current } \\
\text { size } \\
(\%)\end{array}$ & $\begin{array}{c}\text { Power } \\
\text { Sample } \\
\text { size = 300 } \\
(\%)\end{array}$ & $\begin{array}{c}\text { Power } \\
\text { Sample } \\
\text { size }=500 \\
(\%)\end{array}$ & $\begin{array}{c}\text { Power } \\
\text { Sample } \\
\text { size = } \\
1000 \\
(\%)\end{array}$ \\
\hline $\begin{array}{l}\text { Deliberate Self } \\
\text { Harm }\end{array}$ & 196 & $\begin{array}{c}72.73 \\
(16 / 22)\end{array}$ & $\begin{array}{c}55.17 \\
(96 / 174)\end{array}$ & 17.0 & 100 & 100 & 100 & 100 \\
\hline
\end{tabular}


Table 3D: Penetration by an object

\begin{tabular}{|c|c|c|c|c|c|c|c|c|}
\hline Aggressive Behavior & $\begin{array}{c}\text { Current } \\
\text { Sample } \\
\text { size }\end{array}$ & $\begin{array}{c}\text { Victimized } \\
\text { Males } \\
\% \text { (n/Total } \\
\text { male Victims) }\end{array}$ & $\begin{array}{l}\text { Victimized } \\
\text { Females } \\
\% \text { (n/Total } \\
\text { female } \\
\text { victims) }\end{array}$ & $\begin{array}{c}\% \\
\text { Difference }\end{array}$ & $\begin{array}{c}\text { Power } \\
\text { With } \\
\text { Sample } \\
\text { current } \\
\text { size } \\
(\%)\end{array}$ & $\begin{array}{c}\text { Power } \\
\text { Sample } \\
\text { size }= \\
\mathbf{3 0 0} \\
(\%)\end{array}$ & $\begin{array}{c}\text { Power } \\
\text { Sample } \\
\text { size }= \\
\mathbf{5 0 0} \\
(\%)\end{array}$ & $\begin{array}{c}\text { Power } \\
\text { Sample } \\
\text { size = } \\
\mathbf{1 0 0 0} \\
(\%)\end{array}$ \\
\hline Deliberate Self Harm & 203 & $\begin{array}{c}60.98 \\
(25 / 41) \\
\end{array}$ & $\begin{array}{c}58.02 \\
(94 / 162) \\
\end{array}$ & 2.96 & 21.2 & 27 & 37.9 & 60.1 \\
\hline
\end{tabular}

Table 3E: Sexual Intercourse

\begin{tabular}{|c|c|c|c|c|c|c|c|c|}
\hline Aggressive Behavior & $\begin{array}{c}\text { Current } \\
\text { Sample } \\
\text { size }\end{array}$ & $\begin{array}{c}\text { Victimized } \\
\text { Males } \\
\% \text { (n/Total } \\
\text { male Victims) }\end{array}$ & $\begin{array}{c}\text { Victimized } \\
\text { Females } \\
\% \text { (n/Total } \\
\text { female } \\
\text { victims) }\end{array}$ & $\begin{array}{c}\% \\
\text { Difference }\end{array}$ & $\begin{array}{l}\text { Power } \\
\text { With } \\
\text { Sample } \\
\text { current } \\
\text { size } \\
(\%) \\
\end{array}$ & $\begin{array}{c}\text { Power } \\
\text { Sample } \\
\text { size }= \\
300 \\
(\%)\end{array}$ & $\begin{array}{c}\text { Power } \\
\text { Sample } \\
\text { size }= \\
\mathbf{5 0 0} \\
(\%)\end{array}$ & $\begin{array}{c}\text { Power } \\
\text { Sample } \\
\text { size }= \\
1000 \\
(\%)\end{array}$ \\
\hline Deliberate Self Harm & 240 & $\begin{array}{c}55.32 \\
(26 / 47)\end{array}$ & $\begin{array}{c}57.51 \\
(111 / 193)\end{array}$ & 2.19 & 17 & 19.1 & 25.8 & 40.4 \\
\hline
\end{tabular}

\section{- Hypothesis 2.4: Victimized males are at a greater risk of suicidal behavior when compared with victimized females.}

Comparison Groups: Victimized males vs. victimized females

Outcome Measure: Suicidal tendency

Table 4A: Sexual Contact

\begin{tabular}{|c|c|c|c|c|c|c|c|c|}
\hline Aggressive Behavior & $\begin{array}{c}\text { Current } \\
\text { Sample } \\
\text { size }\end{array}$ & $\begin{array}{c}\text { Victimized } \\
\text { Males } \\
\% \text { (n/Total } \\
\text { male Victims) }\end{array}$ & $\begin{array}{l}\text { Victimized } \\
\text { Females } \\
\% \text { (n/Total } \\
\text { female } \\
\text { victims) }\end{array}$ & $\begin{array}{c}\% \\
\text { Difference }\end{array}$ & $\begin{array}{c}\text { Power } \\
\text { With } \\
\text { Sample } \\
\text { current } \\
\text { size } \\
(\%)\end{array}$ & $\begin{array}{c}\text { Power } \\
\text { Sample } \\
\text { size }= \\
300 \\
(\%)\end{array}$ & $\begin{array}{c}\text { Power } \\
\text { Sample } \\
\text { size = } \\
\mathbf{5 0 0} \\
(\%)\end{array}$ & $\begin{array}{c}\text { Power } \\
\text { Sample } \\
\text { size }= \\
1000 \\
(\%)\end{array}$ \\
\hline Suicidal tendency & 285 & $\begin{array}{c}7.14 \\
(3 / 42)\end{array}$ & $\begin{array}{c}10.29 \\
(25 / 243)\end{array}$ & 3.15 & 54.9 & $\begin{array}{l}57.1 \\
\end{array}$ & 78.7 & $\begin{array}{l}97.3 \\
\end{array}$ \\
\hline
\end{tabular}

Table 4B: Coercion

\begin{tabular}{|c|c|c|c|c|c|c|c|c|}
\hline Aggressive Behavior & $\begin{array}{c}\text { Current } \\
\text { Sample } \\
\text { size }\end{array}$ & $\begin{array}{c}\text { Victimized } \\
\text { Males } \\
\% \text { (n/Total } \\
\text { male Victims) }\end{array}$ & $\begin{array}{c}\text { Victimized } \\
\text { Females } \\
\% \text { (n/Total } \\
\text { female } \\
\text { victims) }\end{array}$ & $\begin{array}{c}\% \\
\text { Difference }\end{array}$ & $\begin{array}{c}\text { Power } \\
\text { With } \\
\text { Sample } \\
\text { current } \\
\text { size } \\
(\%) \\
\end{array}$ & $\begin{array}{c}\text { Power } \\
\text { Sample } \\
\text { size }= \\
300 \\
(\%)\end{array}$ & $\begin{array}{c}\text { Power } \\
\text { Sample } \\
\text { size }= \\
500 \\
(\%)\end{array}$ & $\begin{array}{c}\text { Power } \\
\text { Sample } \\
\text { size }= \\
1000 \\
(\%)\end{array}$ \\
\hline Suicidal tendency & 184 & $\begin{array}{c}9.52 \\
(2 / 21)\end{array}$ & $\begin{array}{c}14.11 \\
(23 / 163)\end{array}$ & 4.59 & 56.8 & 77.6 & 93.9 & 99.9 \\
\hline
\end{tabular}

Table 4C: Attempted Sexual Intercourse

\begin{tabular}{|c|c|c|c|c|c|c|c|c|}
\hline Aggressive Behavior & $\begin{array}{c}\text { Current } \\
\text { Sample } \\
\text { size }\end{array}$ & $\begin{array}{c}\text { Victimized } \\
\text { Males } \\
\% \text { (n/Total } \\
\text { male Victims) }\end{array}$ & $\begin{array}{l}\text { Victimized } \\
\text { Females } \\
\% \text { (n/Total } \\
\text { female } \\
\text { victims) }\end{array}$ & $\begin{array}{c}\% \\
\text { Difference }\end{array}$ & $\begin{array}{c}\text { Power } \\
\text { With } \\
\text { Sample } \\
\text { current } \\
\text { size } \\
(\%) \\
\end{array}$ & $\begin{array}{c}\text { Power } \\
\text { Sample } \\
\text { size }= \\
300 \\
(\%)\end{array}$ & $\begin{array}{c}\text { Power } \\
\text { Sample } \\
\text { size }= \\
\mathbf{5 0 0} \\
(\%)\end{array}$ & $\begin{array}{c}\text { Power } \\
\text { Sample } \\
\text { size }= \\
1000 \\
(\%)\end{array}$ \\
\hline Suicidal tendency & 200 & $\begin{array}{l}13.04 \\
(3 / 23)\end{array}$ & $\begin{array}{c}11.86 \\
(21 / 177)\end{array}$ & 1.18 & 13.9 & 16.5 & 21.3 & 31.9 \\
\hline
\end{tabular}


Table 4D: Penetration with an object

\begin{tabular}{|c|c|c|c|c|c|c|c|c|}
\hline Aggressive Behavior & $\begin{array}{c}\text { Current } \\
\text { Sample } \\
\text { size }\end{array}$ & $\begin{array}{c}\text { Victimized } \\
\text { Males } \\
\% \text { (n/Total } \\
\text { male Victims) }\end{array}$ & $\begin{array}{l}\text { Victimized } \\
\text { Females } \\
\% \text { (n/Total } \\
\text { female } \\
\text { victims) }\end{array}$ & $\begin{array}{c}\% \\
\text { Difference }\end{array}$ & $\begin{array}{c}\text { Power } \\
\text { With } \\
\text { Sample } \\
\text { current } \\
\text { size } \\
(\%) \\
\end{array}$ & $\begin{array}{c}\text { Power } \\
\text { Sample } \\
\text { size }= \\
\mathbf{3 0 0} \\
(\%)\end{array}$ & $\begin{array}{c}\text { Power } \\
\text { Sample } \\
\text { size = } \\
\mathbf{5 0 0} \\
(\%)\end{array}$ & $\begin{array}{c}\text { Power } \\
\text { Sample } \\
\text { size }= \\
1000 \\
(\%)\end{array}$ \\
\hline Suicidal tendency & 203 & $\begin{array}{c}7.32 \\
(3 / 41)\end{array}$ & $\begin{array}{c}10.49 \\
(17 / 162)\end{array}$ & 3.17 & 42 & 56.9 & 78.4 & 97.2 \\
\hline
\end{tabular}

Table 4E: Sexual Intercourse

\begin{tabular}{|c|c|c|c|c|c|c|c|c|}
\hline $\begin{array}{l}\text { Aggressive } \\
\text { Behavior }\end{array}$ & $\begin{array}{l}\text { Current } \\
\text { Sample } \\
\text { size }\end{array}$ & $\begin{array}{c}\text { Victimized } \\
\text { Males } \\
\%(n / \text { Total } \\
\text { male Victims) }\end{array}$ & $\begin{array}{l}\text { Victimized } \\
\text { Females } \\
\% \text { (n/Total } \\
\text { female } \\
\text { victims) }\end{array}$ & \% Difference & $\begin{array}{c}\text { Power } \\
\text { With } \\
\text { Sample } \\
\text { current } \\
\text { size } \\
(\%) \\
\end{array}$ & $\begin{array}{c}\text { Power } \\
\text { Sample } \\
\text { size = 300 } \\
(\%)\end{array}$ & $\begin{array}{c}\text { Power } \\
\text { Sample } \\
\text { size = 500 } \\
(\%)\end{array}$ & $\begin{array}{c}\text { Power } \\
\text { Sample } \\
\text { size }= \\
1000 \\
(\%)\end{array}$ \\
\hline Suicidal tendency & 240 & $\begin{array}{c}6.38 \\
(3 / 47)\end{array}$ & $\begin{array}{c}12.44 \\
(24 / 193)\end{array}$ & 6.06 & 94.7 & 100 & 100 & 100 \\
\hline
\end{tabular}




\section{References}




\section{References}

1. Basile KC, Saltzman LE. Sexual violence surveillance: Uniform definitions and recommended data elements. Atlanta, GA: National Center for Injury Prevention and Control, Centers for Disease Control and Prevention; 2002.

2. American Medical Association. Strategies for the treatment and prevention of sexual assault. Chicago, IL: AMA; 1995.

3. Abbey A. Alcohol-related sexual assault: a common problem among college students. Journal of Studies on Alcohol. Supplement. Mar 2002(14):118-128.

4. Tjaden P, Thoennes N. Extent, Nature, and Consequences of Rape Victimization: Findings From the National Violence Against Women Survey. Washington DC: U.S. Department of Justice, National Institute of Justice; 2006. NCJ 210346.

5. Isely PJ. Sexual Assault of Men: College-Age Victims. NASPA Journal. 1998;35(4):305317.

6. Rentoul L, Appleboom N. Understanding the psychological impact of rape and serious sexual assault of men: a literature review. Journal of Psychiatric and Mental Health Nursing Aug 1997;4(4):267-274.

7. Walker J, Archer J, Davies M. Effects of male rape on psychological functioning. British Journal of Clinical Psychology. 2005;44(3):445-451.

8. Stermac L, Del Bove G, Addison M. Stranger and Acquaintance Sexual Assault of Adult Males. Journal of Interpersonal Violence. 2004;19(8):901-915.

9. Stolzenberg L, D'Alessio SJ. Sex differences in the likelihood of arrest. Journal of Criminal Justice. 2004;32(5):443-454. 
10. Kassing LR, Prieto LR. The Rape Myth and Blame-Based Beliefs of Counselors-inTraining Toward Male Victims of Rape. Journal of Counseling \& Development. 2003;81(4):455-461.

11. Basile KC, Chen J, Black MC, Saltzman LE. Prevalence and characteristics of sexual violence victimization among U.S. adults, 2001-2003. Violence and Victims. 2007;22(4):437-448.

12. Tewksbury R. Effects of sexual assaults on men: Physical, mental and sexual consquences. International Journal of Men's Health. 2007;6(1):22-35.

13. Coxell A, King M, Mezey G, Gordon D. Lifetime prevalence, characteristics, and associated problems of non-consensual sex in men: cross sectional survey. British Medical Journal. Mar 27 1999;318(7187):846-850.

14. Stermac L, Sheridan PM, Davidson A, Dunn S. Sexual assault of adult males. Journal of Interpersonal Violence. 1996;11(1):52-64.

15. Isely PJ, Gehrenbeck-Shim D. Sexual assault of men in the community. Journal of Community Psychology. 1997;25(2):159-166.

16. Comstock GD. Victims of anti-gay/lesbian violence. Journal of Interpersonal Violence. 1989;4(1):101-106.

17. Davis M. Male sexual assault victims: A selective review of the literature and implications for support services. Aggressive and Violent Behavior. 2002;7:203-214.

18. Ratner PA, Johnson JL, Shoveller JA, et al. Non-consensual sex experienced by men who have sex with men: prevalence and association with mental health. Patient Education and Counseling. Jan 2003;49(1):67-74.

19. King M, Woollett E. Sexually assaulted males: 115 men consulting a counseling service. Archives of Sexual Behavior. Dec 1997;26(6):579-588. 
20. Mezey G, King M. The effects of sexual assault on men: A survey of 22 victims. Psychological Medicine. 1989;19(1):205-209.

21. Walker J, Archer J, Davies M. Effects of rape on men: a descriptive analysis. Archives of Sexual Behavior. Feb 2005;34(1):69-80.

22. Davies M, Pollard P, Archer J. Effects of perpetrator gender and victim sexuality on blame toward male victims of sexual assault. Journal of Social Psychology. 2006;146(3):275-291.

23. Struckman-Johnson D, Struckman-Johnson C. Men and women's acceptance of coercive sexual strategies varied by initiator gender and couple intimacy. Sex Roles. Dec 1991;25(11-12):661-676.

24. Lott B, Reilly ME, Howard DR. Sexual assault and harassment: A campus community case study. Signs. 1982;8(2):296-319.

25. Aizenman M, Kelley G. The Incidence of violence and acquaintance rape in dating relationships among college men and women. Journal of College Student Development. 1988;28:305-311.

26. O'Sullivan LF, Byers ES, Finkelman L. A comparison of male and female college students' experiences of sexual coercion. Psychology of Women Quarterly. 1998;22(2):177-195.

27. Smyth JM, Hockemeyer JR, Heron KE, Wonderlich SA, Pennebaker JW. Prevalence, type, disclosure, and severity of adverse life events in college students. Journal of American College Health. 2008;57(1):69-76.

28. Hillman RJ, O'Mara N, Taylor-Robinson D, Harris JR. Medical and social aspects of sexual assault of males: a survey of 100 victims. The British Journal of General Practice Dec 1990;40(341):502-504. 
29. Choudhary E, Coben J, Bossarte R. Adverse Health Outcomes, Perpetrator Characteristics, and Sexual Violence Victimization among U.S. Adult Males. Journal of Interpersonal Violence. 2008;In press.

30. Pesola GR, Westfal RE, Kuffner CA. Emergency department characteristics of male sexual assault. Acad Emerg Med. Aug 1999;6(8):792-798.

31. Struckman-Johnson CJ. Forced sex on dates: it happens to men, too. Journal of Sex Research. 1988;24:2340241.

32. Davies M, Rogers P. Perceptions of male victims in depicted sexual assaults: A review of the literature. Aggression and Violent Behavior. 2006;11(4):367-377.

33. Kite ME, Whitley BE, Jr. Sex differences in attitudes toward homosexual persons, behaviors, and civil rights: A meta-analysis. Personality and Social Psychology Bulletin. 1996;22(4):336-353.

34. Davies M. Male sexual assault victims: A selective review of the literature and implications for support services. Aggressive and Violent Behavior. 2002;7:203-214.

35. Barron JM, Struckman-Johnson C, Quevillon R, Banka SR. Heterosexual men's attitudes toward gay men: A hierarchical model including masculinity, openness, and theoretical explanations. Psychology of Men \& Masculinity. 2008;9(3):154-166.

36. Anderson I. What is a typical rape? Effects of victim and participant gender in female and male rape perception. British Journal of Social Psychology. 2007;46(1):225-245.

37. Romano E, De Luca RV. Evaluation of a Treatment Program for Sexually Abused Adult Males. Journal of Family Violence. 2006;21(1):75-88.

38. Monroe LM, Kinney LM, Weist MD, Dafeamekpor DS, Dantzler J, Reynolds MW. The experience of sexual assault: findings from a statewide victim needs assessment. Journal of Interpersonal Violence. 2005;20(7):767-776. 
39. Frazier PA. A comparative study of male and female rape victims seen at a hospitalbased rape crisis program. Journal of Interpersonal Violence. 1993;8(1):64-76.

40. Leserman J. Sexual abuse history: prevalence, health effects, mediators, and psychological treatment. Psychosomatic Medicine Nov-Dec 2005;67(6):906-915.

41. King M, Coxell A, Mezey G. Sexual molestation of males: Associations with psychological disturbance. British Journal of Psychiatry. 2002;181(2):153-157.

42. Myers MF. Men sexually assaulted as adults and sexually abused as boys. Archives of Sexual Behavior. 1989;18(3):203-215.

43. Lacey HB, Roberts R. Sexual assault on men. International Journal of STD \& AIDS. JulAug 1991;2(4):258-260.

44. Vearnals S, Campbell Ts. Male victims of male sexual assault: A review of psychological consequences and treatment. Sexual and Relationship Therapy. 2001;16(3):279-286.

45. Schwartz IL. Sexual violence against women: Prevalence, consequences, societal factors, and prevention. American Journal of Preventive Medicine. 1991;7(6):363-373.

46. Campbell L, Keegan A, Cybulska B, Forster G. Prevalence of mental health problems and deliberate self-harm in complainants of sexual violence. Journal of Forensic And Legal Medicine. 2007;14(2):75-78.

47. Coker AL, Davis KE, Arias I, et al. Physical and mental health effects of intimate partner violence for men and women. American Journal of Preventive Medicine. Nov 2002;23(4):260-268.

48. Garlow SJ, Rosenberg J, Moore JD, et al. Depression, desperation, and suicidal ideation in college students: Results from the American Foundation for Suicide Prevention College Screening Project at Emory University. Depression and Anxiety. 2008;25(6):482488. 
49. Juon H-S, Ensminger ME. Childhood, adolescent, and young adult predictors of suicidal behaviors: A prospective study of African Americans. Journal of Child Psychology and Psychiatry. 1997;38(5):553-563.

50. Waldrop AE, Hanson RF, Resnick HS, Kilpatrick DG, Naugle AE, Saunders BE. Risk factors for suicidal behavior among a national sample of adolescents: Implications for prevention. Journal of Traumatic Stress. 2007;20(5):869-879.

51. Norlev J, Davidsen M, Sundaram V, Kjoller M. Indicators associated with suicidal ideation and suicide attempts among 16-35-year-old Danes: a national representative population study. Suicide and Life-Threatening Behavior. Jun 2005;35(3):291-308.

52. Masho SW, Ahmed G. Age at sexual assault and posttraumatic stress disorder among women: prevalence, correlates, and implications for prevention. Journal of Women's Health. 2007;16(2):262-271.

53. Gladstone GL, Parker GB, Mitchell PB, Malhi GS, Wilhelm K, Austin M-P. Implications of childhood trauma for depressed women: An analysis of pathways from childhood sexual abuse to deliberate self-harm and revictimization. American Journal of Psychiatry. 2004;161(8):1417-1425.

54. Barrios LC, Everett SA, Simon TR, Brener ND. Suicide ideation among US college students. Associations with other injury risk behaviors. Journal of American College Health. 2000;48(5):229-233.

55. Bronisch T. Depression and Suicidal Behavior. Crisis: The Journal of Crisis Intervention and Suicide Prevention. 2003;24(4):179-180.

56. Ullman SE. Sexual assault victimization and suicidal behavior in women: A review of the literature. Aggression and Violent Behavior. 2004;9(4):331-351.

57. Gatz M, Russell LA, Grady J, Kram-Fernandez D, Clark C, Marshall B. Women's Recollections of Victimization, Psychological Problems, and Substance Use. Journal of Community Psychology. 2005;33(4):479-493. 
58. Briere J, Runtz M. Suicidal thoughts and behaviors in former sexual abuse victims. Canadian Journal of Behavioural Science. 1986;18(4):413-423.

59. Brunner R, Parzer P, Haffner J, et al. Prevalence and psychological correlates of occasional and repetitive deliberate self-harm in adolescents. Archives of Pediatrics \& Adolescent Medicine. Jul 2007;161(7):641-649.

60. Tuisku V, Pelkonen M, Karlsson L, et al. Suicidal ideation, deliberate self-harm behaviour and suicide attempts among adolescent outpatients with depressive mood disorders and comorbid axis I disorders. European Child \& Adolescent Psychiatry. 2006;15(4):199-206.

61. Hawton K, Kingsbury S, Steinhardt K, James A, Fagg J. Repetition of deliberate selfharm by adolescents: The role of psychological factors. Journal of Adolescence. 1999;22(3):369-378.

62. Gratz KL. Measurement of deliberate self-harm: Preliminary data on the Deliberate SelfHarm Inventory. Journal of Psychopathology and Behavioral Assessment. 2001;23(4):253-263.

63. Mangnall J, Yurkovich E. A literature review of deliberate self-harm. Perspectives in Psychiatric Care. 2008;44(3):175-184.

64. Agnew RS, Flannery DJ, Vazsonyi AT, Waldman ID. Strain theory and violent behavior. The Cambridge handbook of violent behavior and aggression. New York, NY US: Cambridge University Press; 2007:519-529.

65. Baron SW. General strain, street youth, and crime: A test of Agnew's revised theory. Criminology. 2004;42(2):457-484.

66. Sorsoli L, Kia-Keating M, Grossman FK. 'I keep that hush-hush': Male survivors of sexual abuse and the challenges of disclosure. Journal of Counseling Psychology. 2008;55(3):333-345. 
67. Garnefski N, Arends E. Sexual abuse and adolescent maladjustment: Differences between male and female victims. Journal of Adolescence. 1998;21(1):99-107.

68. Sigfusdottir ID, Asgeirsdottir BB, Gudjonsson GH, Sigurdsson JF. A model of sexual abuse's effects on suicidal behavior and delinquency: The role of emotions as mediating factors. Journal of Youth and Adolescence. 2008;37(6):699-712.

69. Tewksbury R, Mustaine EE. Lifestyle factors associated with the sexual assault of men: A routine activity theory analysis. The Journal of Men's Studies. 2001;9(2):153-182.

70. Deliramich AN, Gray MJ. Changes in women's sexual behavior following sexual assault. Behavior Modification. 2008;32(5):611-621.

71. Koss MP. Rape. Scope, impact, interventions, and public policy responses. American Psychologist. Oct 1993;48(10):1062-1069.

72. Campbell R. The psychological impact of rape victims. American Psychologist. 2008;63(8):702-717.

73. Rand MR. Criminal Victimization, 2007. Washington, DC. : U.S. Department of Justice, Bureau of Justice Statistics; 2008. NCJ 224390.

74. Coxell A, King M, Mezey G, Gordon D. Lifetime prevalence, characteristics, and associated problems of non-consensual sex in men: cross sectional survey. Bmj. Mar 27 1999;318(7187):846-850.

75. Kaplan DW, Feinstein RA, Fisher MM, et al. Care of the adolescent sexual assault victim. Pediatrics. 2001;107(6):1476-1479.

76. Leserman J. Sexual abuse history: prevalence, health effects, mediators, and psychological treatment. Psychosom Med. Nov-Dec 2005;67(6):906-915.

77. Rentoul L, Appleboom N. Understanding the psychological impact of rape and serious sexual assault of men: a literature review. J Psychiatr Ment Health Nurs. Aug 1997;4(4):267-274. 
78. Finkelhor D, Hotaling G, Lewis IA, Smith C. Sexual abuse in a national survey of adult men and women: prevalence, characteristics, and risk factors. Child Abuse Negl. 1990;14(1):19-28.

79. Holmes WC, Slap GB. Sexual abuse of boys: definition, prevalence, correlates, sequelae, and management. Jama. Dec 2 1998;280(21):1855-1862.

80. Peipert JF, Domagalski LR. Epidemiology of adolescent sexual assault. Obstet Gynecol. Nov 1994;84(5):867-871.

81. Muram D, Hostetler BR, Jones CE, Speck PM. Adolescent victims of sexual assault. Journal of Adolescent Health. 1995;17(6):372-375.

82. Kaufman M, and the Committee on A. Care of the Adolescent Sexual Assault Victim. Pediatrics. 2008;122(2):462-470.

83. Lacey HB, Roberts R. Sexual assault on men. Int J STD AIDS. Jul-Aug 1991;2(4):258260.

84. Abbey A. Alcohol-related sexual assault: a common problem among college students. $J$ Stud Alcohol Suppl. Mar 2002(14):118-128.

85. Young A, Grey M, Abbey A, Boyd CJ, McCabe SE. Alcohol-related sexual assault victimization among adolescents: prevalence, characteristics, and correlates. Journal Of Studies On Alcohol And Drugs. 2008;69(1):39-48.

86. Wolitzky-Taylor KB, Ruggiero KJ, Danielson CK, et al. Prevalence and correlates of dating violence in a national sample of adolescents. J Am Acad Child Adolesc Psychiatry. Jul 2008;47(7):755-762.

87. U.S. Dept. of Justice, Federal Bureau of Investigation. NATIONAL INCIDENT-BASED REPORTING SYSTEM, 2005: EXTRACT FILES [Computer file]. Ann Arbor, MI: Interuniversity Consortium for Political and Social Research [producer and distributor]; 2007. 
88. US Census Bureau. http://www.census.gov/main/www/cen2000.html. Accessed December 12, 2008.

89. McCullagh P, Nelder J. Generalized Linear Models. 2nd ed. London: Chapman and Hall; 1989.

90. SAS/STAT User's Guide. The GENMOD Procedure. Cary, North Carolina: SAS Institute Inc.; 1999.

91. Dunn CS, Zelenock TJ. NIBRS data available for secondary analysis. Journal of Quantitative Criminology. 1999;15(2):239-248.

92. Larimer ME, Lydum AR, Anderson BK, Turner AP. Male and female recipients of unwanted sexual contact in a college student sample: Prevalence rates, alcohol use, and depression symptoms. Sex Roles. 1999;40(3):295-308.

93. Catalano SM. Criminal Victimization, 2003. Washington, DC: U.S. Department of Justice. Bureau of Justice Statistics; 2004. NCJ 205455.

94. Catalano SM. Criminal Victimization, 2004. Washington, DC: U.S. Department of Justice. Bureau of Justice Statistics; 2005. NCJ 210674.

95. Catalano SM. Criminal Victimization, 2005. Washington, DC: U.S. Department of Justice. Bureau of Justice Statistics; 2006. NCJ 214644.

96. Rennison C. Criminal Victimization 2001: Changes 2000-01 with Trends 1993-2001. Washington, DC: U.S. Department of Justice. Bureau of Justice Statistics; 2002. NCJ 194610.

97. Rennison C, Rand MR. Criminal Victimization, 2002. Washington, DC: U.S. Department of Justice. Bureau of Justice Statistics; 2003. NCJ 199994.

98. Hillman RJ, O'Mara N, Taylor-Robinson D, Harris JR. Medical and social aspects of sexual assault of males: a survey of 100 victims. Br J Gen Pract. Dec 1990;40(341):502504. 
99. Ellis CD. Male rape--the silent victims. Collegian. Oct 2002;9(4):34-39.

100. Sable MR, Danis F, Mauzy DL, Gallagher SK. Barriers to Reporting Sexual Assault for Women and Men: Perspectives of College Students. Journal of American College Health. 2006;55(3):157-162.

101. Snyder HN. Sexual Assault of Young Children as Reported to Law enforcemnt: Victim, Incident, and Offender Characteristics. Washington, DC: U.S. Department of Justics, Bureau of Justice Statistics; 2000. NCJ 182990.

102. Johnson CF. Child sexual abuse. The Lancet.364(9432):462-470.

103. Finkelhor D, Ormrod RK, Turner HA. The Developmental Epidemiology of Childhood Victimization. J Interpers Violence. May 82008.

104. MacMillan HL, Fleming JE, Streiner DL, et al. Childhood abuse and lifetime psychopathology in a community sample. American Journal of Psychiatry. 2001;158(11):1878-1883.

105. Hanson RF, Borntrager C, Self-Brown S, et al. Relations among gender, violence exposure, and mental health: The national survey of adolescents. American Journal of Orthopsychiatry. 2008;78(3):313-321.

106. Roy A. Childhood trauma and attempted suicide in alcoholics. Journal of Nervous and Mental Disease. 2001;189(2):120-121.

107. Fields SD, Malebranche D, Feist-Price S. Childhood sexual abuse in black men who have sex with men: Results from three qualitative studies. Cultural Diversity and Ethnic Minority Psychology. 2008;14(4):385-390.

108. Plant M, Miller P, Plant M. Childhood and Adult Sexual Abuse: Relationships with Alcohol and other Psychoactive Drug Use. Child Abuse Review. 2004;13(3):200-214. 
109. Sansone RA, Gaither GA, Songer DA. Self-harm behaviors and mental healthcare utilization among sexually abused males: A pilot study. General Hospital Psychiatry. 2001;23(2):97-98.

110. Stein MB, Barrett-Connor E. Sexual Assault and Physical Health: Findings From a Population-Based Study of Older Adults. Vol 62; 2000:838-843.

111. D'Augelli AR, Grossman AH. Disclosure of Sexual Orientation, Victimization, and Mental Health Among Lesbian, Gay, and Bisexual Older Adults. Vol 16; 2001:10081027.

112. Chang BH, Skinner KM, Zhou C, Kazis LE. The relationship between sexual assault, religiosity, and mental health among male veterans. Int J Psychiatry Med. 2003;33(3):223-239.

113. Choudhary E, Coben J, Bossarte R. Adverse Health Outcomes, Perpetrator Characteristics, and Sexual Violence Victimization among U.S. Adult Males. Journal of Interpersonal Violence. 2008;Under Review.

114. McCloskey KA, Raphael DN. Adult perpetrator gender asymmetries in child sexual assault victim selection: results from the 2000 National Incident-Based Report System. Journal Of Child Sexual Abuse. 2005;14(4):1-24.

115. Tjaden $P$, Thoennes N. Prevalence and consequences of male-to-female and female-tomale intimate partner violence as measured by the National Violence Against Women Survey. Violence Against Women. 2000;6(2):142-161.

116. Struckman-Johnson C, Struckman-Johnson D. Men pressured and forced into sexual experience. Archives of Sexual Behavior. 1994;23(1):93-114.

117. Koons-Witt BA, Schram PJ. The prevalence and nature of violent offending by females. Journal of Criminal Justice. 2003;31(4):361-371.

118. Pazzani LM. The factors affecting sexual assaults committed by strangers and acquaintances. Violence Against Women. 2007;13(7):717-749. 
119. Chilton R, Jarvis J. Using the National Incident-Based Reporting System (NIBRS) to test estimates of arrestee and offender characteristics. Journal of Quantitative Criminology. 1999;15(2):207-224.

120. Walker J, Archer J, Davies M. Effects of rape on men: a descriptive analysis. Arch Sex Behav. Feb 2005;34(1):69-80.

121. National Incident-Based Reporting System Volume 1: Data Collection Guidelines. Clarksburg, West Virginia: U.S. Department of Justice, Federal Bureau of Investigation, Criminal Justice Information Services Division, Uniform Crime Reporting Program; 2000.

122. Street AE, Gradus JL, Stafford J, Kelly K. Gender differences in experiences of sexual harassment: Data from a male-dominated environment. Journal of Consulting and Clinical Psychology. 2007;75(3):464-474.

123. Masho SW, Odor RK, Adera T. Sexual assault in Virginia: A population-based study. Women's Health Issues. 2005;15(4):157-166.

124. Juhascik MP, Negrusz A, Faugno D, et al. An estimate of the proportion of drugfacilitation of sexual assault in four U.S. localities. Journal Of Forensic Sciences. 2007;52(6):1396-1400.

125. Acierno R, Resnick HS. Health impact of interpersonal violence 1: Prevalence rates, case identification, and risk. Behavioral Medicine. 1997;23(2):53-53.

126. Choudhary E, Coben J, Bossarte R. Gender and time differences in the associations between sexual violence victimization, health outcomes, and risk behaviors. American Journal of Men's Health. 2008;2:254-259.

127. Resick PA. The psychological impact of rape. Journal of Interpersonal Violence. $1993 ; 8(2): 223-255$. 
128. Wolitzky-Taylor KB, Ruggiero KJ, Danielson CK, et al. Prevalence and correlates of dating violence in a national sample of adolescents. Journal of the American Academy of Child and Adolescent Psychiatry. Jul 2008;47(7):755-762.

129. Anderson CL. Males as sexual assault victims: Multiple levels of trauma. Journal of Homosexuality. 1981;7(2):145-162.

130. West Virginia University Research Participation System. http://wvu.sonasystems.com/default.asp. Accessed 24th March, 2009.

131. Kolivas ED, Gross AM. Assessing sexual aggression: Addressing the gap between rape victimization and perpetration prevalence rates. Aggression and Violent Behavior. 2007;12(3):315-328.

132. Koss MP, Oros CJ. Sexual Experiences Survey: A research instrument investigating sexual aggression and victimization. Journal of Consulting and Clinical Psychology. 1982;50(3):455-457.

133. ACHA-NCHA. American College Health Association-National College Health Assessment: Reference Group Executive Summary. Baltimore: American College Health Association; Fall 2005.

134. Kroenke K, Spitzer RL, Williams JB. The PHQ-9: validity of a brief depression severity measure. Journal of General Internal Medicine. 2001;16(9):606-613.

135. Strine TW, Mokdad AH, Balluz LS, Berry JT, Gonzalez O. Impact of depression and anxiety on quality of life, health behaviors, and asthma control among adults in the United States with asthma, 2006. The Journal of Asthma. 2008;45(2):123-133.

136. Fliege H, Kocalevent R-D, Walter OB, et al. Three assessment tools for deliberate selfharm and suicide behavior: Evaluation and psychopathological correlates. Journal of Psychosomatic Research. 2006;61(1):113-121.

137. Linehan MM. Suicidal Behavior Questionnaire. Seattle, WA: University of Washington; 1981:Unpiblished Inventory. 
138. Cotton CR, Peters DK, Range LM. Psychometric properties of the Suicidal Behaviors Questionnaire. Death Studies. 1995;19(4):391-397.

139. Gutierrez PM, Osman A, Kopper BA, Barrios FX, Bagge CL. Suicide risk assessment in a college student population. Journal of Counseling Psychology. 2000;47(4):403-413.

140. Addis M, Linehan MM. Predicting suicidal behavior: psychometric properties of the Suicidal Behaviors Questionnaire. Annual Meeting of the Association for the Advancement of Behavior Therapy. Washington, DC; 1989.

141. Brown G. A review of suicide assessment measures for intervention research with adults and older adults. Bethesda, MD: National Institute of Mental Health; October 10, 2008 2002.

142. Swahn MH, Simon TR, Arias I, Bossarte RM. Measuring sex differences in violence victimization and perpetration within date and same-sex peer relationships. Journal of Interpersonal Violence. Aug 2008;23(8):1120-1138.

143. Wechsler H, Dowdall GW, Davenport A, Rimm EB. A gender-specific measure of binge drinking among college students. American Journal of Public Health. Jul 1995;85(7):982-985.

144. Wechsler H, Dowdall GW, Maenner G, Gledhill-Hoyt J, Lee H. Changes in binge drinking and related problems among American college students between 1993 and 1997. Results of the Harvard School of Public Health College Alcohol Study. Journal of American College Health Sep 1998;47(2):57-68.

145. O'Grady KE, Arria AM, Fitzelle DM, Wish ED. Heavy Drinking and Polydrug Use among College Students. Journal of Drug Issues. 2008;38(2):445-466.

146. Cloutier S, Martin SL, Poole C. Sexual assault among North Carolina women: prevalence and health risk factors. Journal of Epidemiology and Community Health. Apr 2002;56(4):265-271. 
147. Flack W, Jr., Daubman K, Caron M, et al. Risk Factors and Consequences of Unwanted Sex Among University Students: Hooking Up, Alcohol, and Stress Response. Journal of Interpersonal Violence. February 1, 2007 2007;22(2):139-157.

148. Nasta A, Shah B, Brahmanandam S, et al. Sexual Victimization: Incidence, Knowledge and Resource Use among a Population of College Women. Journal of Pediatric and Adolescent Gynecology. 2005;18(2):91-96.

149. Forke C, Myers R, Catallozzi M, Schwarz D. Relationship Violence Among Female and Male College Undergraduate Students. Archives of Pediatrics \& Adolescent Medicine. 2008;162(7):634-641.

150. Chan KL, Straus MA, Brownridge DA, Tiwari A, Leung WC. Prevalence of Dating Partner Violence and Suicidal Ideation Among Male and Female University Students Worldwide. Journal of Midwifery \& Women's Health. 2008;53(6):529-537.

151. Howard DE, Griffin MA, Boekeloo BO. Prevalence and psychosocial correlates of alcohol-related sexual assault among university students. Adolescence. Winter2008 2008;43(172):733-750.

152. Abbey A, McAuslan P, Ross LT. Sexual assault perpetration by college men: The role of alcohol, misperception of sexual intent, and sexual beliefs and experiences. Journal of Social \& Clinical Psychology. 1998;17(2):167-195.

153. The Centers for Disease Control and Prevention, Web-based Injury Statistics Query and Reporting System (WISQARS). http://www.cdc.gov/injury/wisqars/. Accessed March $28,2009$.

154. Koss MP, Gidycz CA, Wisniewski N. The scope of rape: Incidence and prevalence of sexual aggression and victimization in a national sample of higher education students. Journal of Consulting and Clinical Psychology. 1987;55(2):162-170.

155. Fisher B, Cullen F, Turner M. The sexual victimization of college women. Washington, DC: National Institute of Justice and Bureau of Justice Statistics; 2000. NCJ 182369. 
156. Banyard VL, Ward S, Cohn ES, Plante EG, Moorhead C, Walsh W. Unwanted sexual contact on campus: a comparison of women's and men's experiences. Violence and Victims. 2007;22(1):52-70.

157. Abbey A, BeShears R, Clinton-Sherrod AM, McAuslan P. Similarities and differences in women's sexual assault experiences based on tactics used by the perpetrator. Psychology of Women Quarterly. 2004;28(4):323-332.

158. Lehrer JA, Lehrer VL, Lehrer EL, Oyarzun PB. Prevalence of and risk factors for sexual victimization in college women in Chile. International Family Planning Perspectives. Dec 2007;33(4):168-175.

159. Choudhary E, Coben J, Bossarte R. Adverse health outcomes, perpetrator characteristics, and sexual violence victimization among U.S. adult males. Journal of Interpersonal Violence. 2009; In press.

160. Koss MP, Oros CJ. Sexual Experiences Survey: a research instrument investigating sexual aggression and victimization. Journal of Consulting and Clinical Psychology. Jun $1982 ; 50(3): 455-457$.

161. Koss MP, Gidycz CA. Sexual Experiences Survey: Reliability and validity. Journal of Consulting and Clinical Psychology. 1985;53(3):422-423.

162. Strauss AL. Qualitative analysis for social scientists. New York, NY US: Cambridge University Press; 1987.

163. Glaser B. Basics of grounded theory analysis. Mill Valley, CA: Sociology Press; 1992.

164. Hsieh HF, Shannon SE. Three approaches to qualitative content analysis. Qualitative Health Research. Nov 2005;15(9):1277-1288.

165. Chapleau KM, Oswald DL, Russell BL. Male rape myths: The role of gender, violence, and sexism. Journal of Interpersonal Violence. 2008;23(5):600-615. 
166. Light D, Monk-Turner E. Circumstances Surrounding Male Sexual Assault and Rape: Findings From the National Violence Against Women Survey. Journal of Interpersonal Violence. 2008:doi:10.1177/0886260508325488.

167. Oswald DL, Russell BL. Perceptions of sexual coercion in heterosexual dating relationships: The role of aggressor gender and tactics. Journal of Sex Research. 2006;43(1):87-95.

168. Russell BL, Oswald DL. Sexual coercion and victimization of college men: The role of love styles. Journal of Interpersonal Violence. 2002;17(3):273-285.

169. Sandberg G, Jackson TL, Petretic-Jackson P. College students' attitudes regarding sexual coercion and aggression: Developing educational and preventive strategies. Journal of College Student Personnel. 1987;28(4):302-311.

170. Gilligan C. In a different voice: Psychological theory and women's development. Cambridge, MA US: Harvard University Press; 1982.

171. Gilligan C, Attanucci J. Two moral orientations: Gender differences and similarities. Merrill-Palmer Quarterly. 1988;34(3):223-237.

172. Gilligan C, Puka B. In a different voice: Women's conceptions of self and of morality. Caring voices and women's moral frames: Gilligan's view. New York, NY US: Garland Publishing; 1994:1-37.

173. Darves-Bornoz JM, Choquet M, Ledoux S, Gasquet I, Manfredi R. Gender differences in symptoms of adolescents reporting sexual assault. Social Psychiatry and Psychiatric Epidemiology. 1998;33(3):111-117.

174. Finkelhor D, Hotaling G, Lewis IA, Smith C. Sexual abuse in a national survey of adult men and women: prevalence, characteristics, and risk factors. Child Abuse and Neglect. 1990;14(1):19-28. 
175. Hayslip B, Jr., Neumann CS, Louden L, et al. Developmental Stage Theories. Comprehensive Handbook of Personality and Psychopathology, Vol. 1: Personality and Everyday Functioning. Hoboken, NJ US: John Wiley \& Sons Inc; 2006:115-141.

176. U. S. Department of Health and Human Services. Health People 2010: With Understanding and Improving Health and Objective for Improving Health (2nd ed) Vol 2. . Washington, DC: U.S. Government Printing Office; November 20002000.

177. Testa M, VanZile-Tamsen C, Livingston JA, Koss MP. Assessing women's experiences of sexual aggression using the sexual experiences survey: Evidence for validity and implications for research. Psychology of Women Quarterly. 2004;28(3):256-265.

178. Gidycz CA, Loh C, Lobo T, Rich C, Lynn SJ, Pashdag J. Reciprocal relationships among alcohol use, risk perception, and sexual victimization: a prospective analysis. Journal of American College Health. 2007;56(1):5-14. 